University of South Carolina

Scholar Commons

Theses and Dissertations

Summer 2021

\title{
A Brief Mindfulness-Based Intervention (bMBI) to Reduce Teacher Stress and Burnout
}

Stephen George Taylor

Follow this and additional works at: https://scholarcommons.sc.edu/etd

Part of the Clinical Psychology Commons, and the Community Psychology Commons

\section{Recommended Citation}

Taylor, S. G.(2021). A Brief Mindfulness-Based Intervention (bMBI) to Reduce Teacher Stress and Burnout. (Doctoral dissertation). Retrieved from https://scholarcommons.sc.edu/etd/6470

This Open Access Dissertation is brought to you by Scholar Commons. It has been accepted for inclusion in Theses and Dissertations by an authorized administrator of Scholar Commons. For more information, please contact digres@mailbox.sc.edu. 
A Brief Mindfulness-Based Intervention (bMBI) to Reduce Teacher Stress and Burnout by

Stephen George Taylor

Bachelor of Science

The Pennsylvania State University, 2015

Master of Arts

University of South Carolina, 2018

Submitted in Partial Fulfillment of the Requirements

For the Degree of Doctor of Philosophy in

Clinical-Community Psychology

College of Arts and Sciences

University of South Carolina

2020

Accepted by:

Nicole Zarrett, Major Professor

M. Michele Burnette, Committee Member

Dawn K. Wilson, Committee Member

Matthew Irvin, Committee Member

Cheryl L. Addy, Vice Provost and Dean of the Graduate School 
(C) Copyright by Stephen George Taylor, 2020

All Rights Reserved. 


\section{DEDICATION}

This project is dedicated to my family, friends, and all the educators whose effort and inspiration helped to shape me throughout my life. 


\section{ACKNOWLEDGEMENTS}

I thank my wife, Angie, for continuing to encourage and support me as I pursue my passion from which I continue to derive abundant meaning and purpose. Her mere presence perpetually reorients me to what matters as all else fades into the background. I thank my parents and sister for their love and guidance throughout my entire life. They will truly never know how much this has impacted me. I thank my friends for their ability to draw me into the present and share in my moments of joy. I thank Alex, my co-PI for this project and the other half of our indomitable duo, whose scrupulousness helped to make this project something we can both be proud of. I thank Nicole Zarrett for her patience and all the knowledge she has passed on to me over the last five years. She has undeniably helped me to improve as both a scholar and a person. I also thank the rest my committee, Michele Burnette, Dawn Wilson, and Matthew Irvin, for all their insight and effort on this project. I thank the educators who volunteered their valuable time to participate in this project and blindly trusted in us to guide them on their own journeys of continual self-improvement. Finally, this research was supported in part by the Office of Executive Vice President for Academic Affairs and Provost and the Office for the Vice President for Research, so I would be remiss if I neglected to thank them for making available the resources required for such a project. 


\begin{abstract}
Teachers are exposed to a variety of chronic stressors in their work environments that lead to stress, burnout, and the deterioration of physiological systems that promote adaptive responses to stress. The downstream effects of chronic stress and burnout incur substantial costs associated with attrition and stress-related health concerns. Research demonstrates that mindfulness-based interventions (MBIs) have potential to improve teachers' capacity to manage stress and mitigate its detrimental effects. However, many MBI studies to date have failed to incorporate key elements of methodological rigor and included large dosages despite research suggesting that such dosages are iatrogenic. Furthermore, these studies have not considered what mechanisms account for positive changes seen in teacher outcomes. The current study examined the efficacy of a randomized waitlist-controlled brief $\mathrm{MBI}(\mathrm{bMBI})$ in a sample of secondary school teachers $(N=23$; four sessions and six hours) utilizing both self-reported and physiological measures of stress (i.e., cortisol awakening response [CAR]). Results indicate that teachers receiving the bMBI demonstrated reductions in self-reported stress, burnout, and depression from pre- to post-intervention despite having a small sample size, which indicates that the impact of the intervention for these outcomes was particularly robust. There were no observed changes in the waitlist-control group. A qualitative assessment of CAR values suggests that teachers in the intervention group were more likely to experience an adaptive shift in physiological functioning while teachers in the control group were more likely to experience a maladaptive shift.
\end{abstract}


Analyses to identify mechanisms of change indicate there were medium effects for total mindfulness and the describe and act with awareness dimensions of mindfulness in the intervention group but not the waitlist-control group. Furthermore, the moderate correlations observed across several dimensions of mindfulness on teacher outcome variables provides insight into dimensions of mindfulness that were most impactful in producing these positive outcomes and are discussed in the context of designing costeffective MBIs that can be tailored to meet the specific needs of various subgroups of teachers. The study highlights the need for future MBIs to reduce intervention dosages to maximize cost-effectiveness and provides directions to continue building on this critical research avenue. 


\section{TABLE OF CONTENTS}

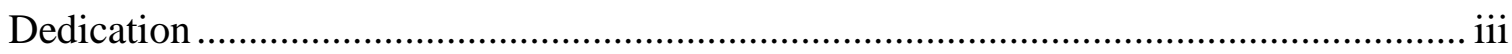

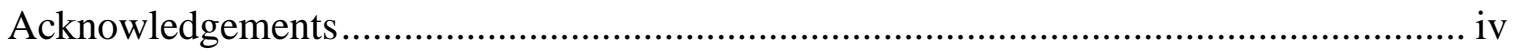

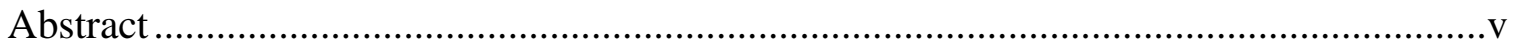

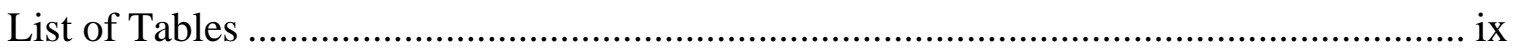

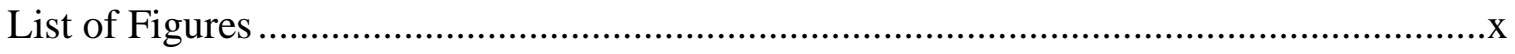

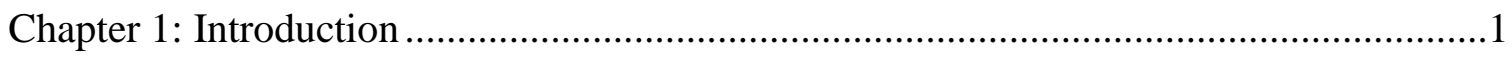

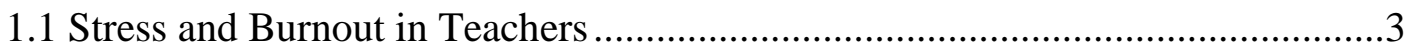

1.2 Costs of Chronic Stress and Burnout ..........................................................

1.3 Mindfulness: Theory and Intervention for Teachers ..................................10

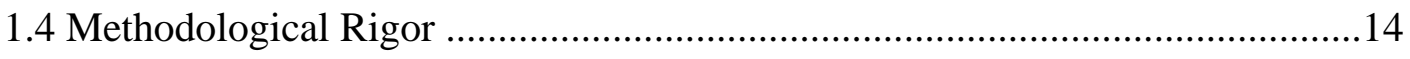

1.5 Efficacy of Mindfulness-Based Interventions for Teachers ............................15

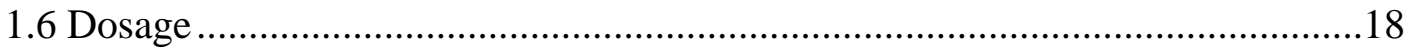

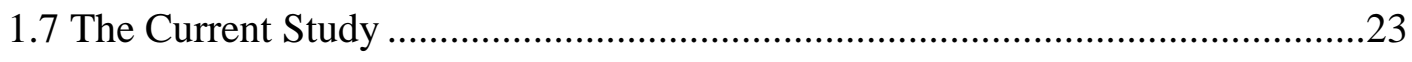

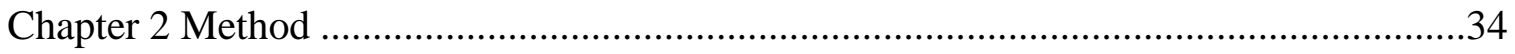

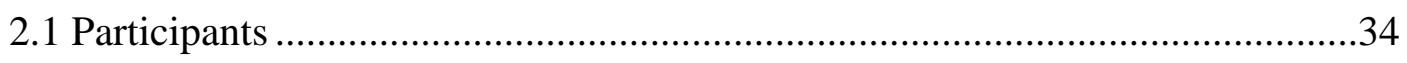

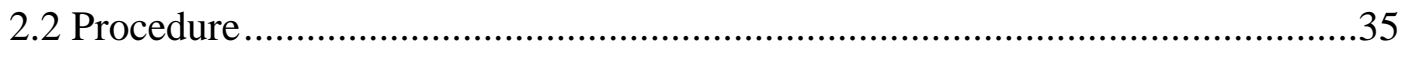

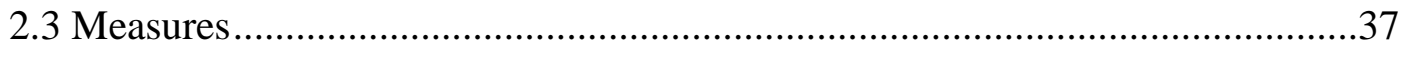

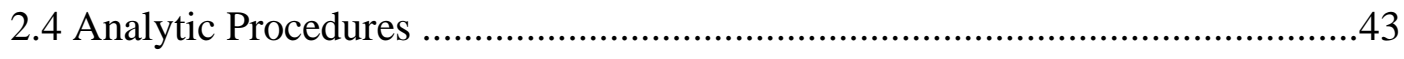

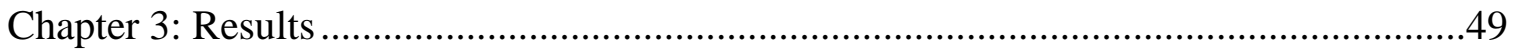

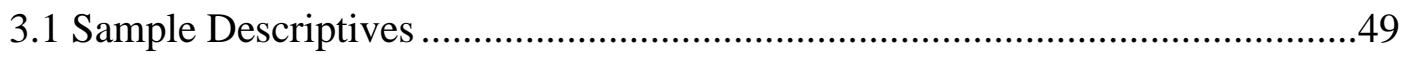


3.2 Aim 1: Efficacy of bMBI on Teacher Stress and Burnout .............................50

3.3 Aim 2: Dimensions of Mindfulness as Mechanisms of Change ......................55

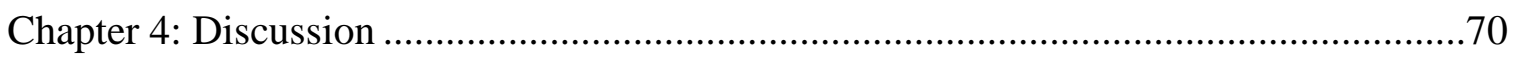

4.1 Impact of the Intervention on Stress, Burnout and Psychological

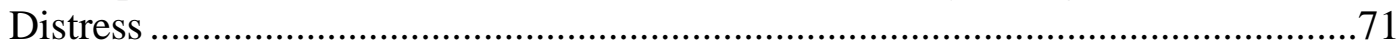

4.2 Mindfulness as Mechanisms of Change .....................................................79

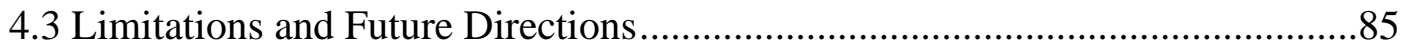

4.4 Conclusion ....................................................................................... 90

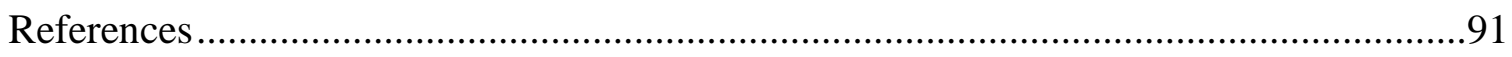

Appendix A: Intervention Outcomes Measures....................................................113

Appendix B: Mechanisms of Change Measure .................................................... 118 


\section{LIST OF TABLES}

Table 1.1 Details of previous MBI studies for teachers.................................................26

Table 1.2 Methodological rigor of reviewed studies ................................................32

Table 2.1 Sociodemographic characteristics of the current study by condition ................46

Table 2.2 Overview of content and key themes in bMBI curriculum ............................47

Table 3.1 Chi square analyses for cortisol awakening response...................................58

Table 3.2 Correlations between mindfulness and primary outcome variables at post-

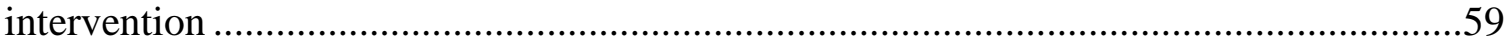

Table 3.3 Dimensions of mindfulness as mechanisms of change .................................61

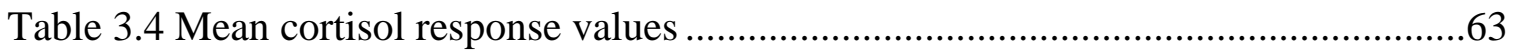

Table 3.5 Shifts in teachers' CAR from pre- to post-intervention ..................................64

Table 3.6 Mean comparisons of teacher outcome variables by group ............................65

Table 3.7 Mean comparisons of teacher mindfulness by group .................................67 


\section{LIST OF FIGURES}

Figure 3.1 Participant cortisol awakening response (CAR) values at pre- and postintervention . .68

Figure 3.2 Participant salivary cortisol values at waking and 30 minutes

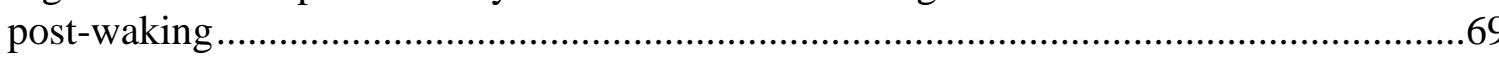




\section{CHAPTER 1}

\section{INTRODUCTION}

Teaching has been identified as a highly stressful occupation (Smith et al., 2000) driven by consistent attentional control and executive functioning demands (Travers, 2001; Roeser, Skinner, Beers, \& Jennings, 2012; Roeser et al., 2013; McCarthy \& Lambert, 2006). The chronic nature of these stressors can lead teachers beyond their coping capacity and result in burnout (Selye, 1956; Lazarus \& Folkman, 1984; Maslach \& Jackson, 1981; Maslach, Jackson, \& Leiter, 1996; Jennett, Harris, \& Mesibov, 2003; LeCompte \& Dworkin, 1991) and a breakdown of physiological systems (i.e., allostatic load; McEwen, 1998; Seeman, Singer, Rowe, Horwitz, \& McEwen, 1997; Bellingrath \& Kudielka, 2017). Leading to emotional exhaustion, reduced teaching efficacy, and low job satisfaction (McCarthy, Lambert, O’Donnell, \& Melendres, 2009; Klassen \& Chiu, 2010), stress and burnout contribute to teacher attrition (Whipp, Tan, \& Yeo, 2007) with approximately $40 \%$ of teachers discontinuing teaching after five years (Ingersoll, 2002). Furthermore, there are considerable costs associated with managing negative physical and mental health outcomes associated with stress, burnout, and subsequent allostatic load (Lopez, Mathers, Ezzati, Jamison, and Murray, 2006; Rice, 1999; McEwen, 1998; Mattei, Demissie, Falcon, Ordovas, \& Tucker, 2010). Many of the interventions designed to reduce teacher stress and burnout have been only marginally successful (Klingbeil \& Renshaw, 2018). The majority of these interventions have taken a person-centered approach to increase coping skills and capacity through cognitive-behavioral strategies 
(e.g., emphasizing time-management and cognitive restructuring; Żołnierczyk-Zreda, 2005; Awa, Plaumann, \& Walter, 2010) with only a subset of these teacher education programs focused on directly facilitating "higher order" skills conducive to successfully coping with stressful vocational-specific demands (Roeser et al., 2012).

Over the past decade, mindfulness-based interventions (MBIs) have become increasingly recognized as an effective intervention to foster these higher-order skills for promoting health and well-being (i.e., stress, internalizing symptomology, etc.; Grossman, Neimann, Schmidt, \& Walach, 2004; Carmody \& Baer, 2008; Roeser et al., 2012) across numerous non-clinical adult populations. In particular, the few that have implemented MBIs for teachers have shown promise in increasing mindfulness skills as a means of reducing occupational stress and symptoms of burnout (Roeser et al., 2012). However, among these teacher-focused MBI studies, there has been significant variability in intervention dosage, with the majority of interventions erring on the side of being too long (i.e., too many direct contact hours), potentially reducing intervention effectiveness (Emerson et al., 2017; Klingbeil \& Renshaw, 2018). Moreover, few studies have collected physiological measures of stress and burnout (Harris, Jennings, Katz, Abenavoli, \& Greenberg, 2016; Flook, Goldberg, Pinger, Bonus, \& Davidson, 2013; Roeser et al., 2013), and those that employed this more objective measure of stress required large doses (i.e., minimally 21 direct contact hours). There is also a paucity of research regarding the domains of mindfulness that account for reductions in teacher stress and burnout in the context of these interventions, which makes it difficult to identify and promote critical mechanisms of change in order to consolidate MBIs down to smaller dosages while maintaining their effectiveness. The current study sought to 
address these gaps by testing the efficacy of a brief randomized controlled bMBI ( 6 total contact hours) to reduce teacher stress and burnout using both self-reported and physiological (i.e., cortisol awakening response [CAR]) measures in a sample of secondary school teachers. Furthermore, the current study explored the effects of separate dimensions of mindfulness that account for reductions in teachers' stress and burnout in order to inform the development of future MBI studies seeking to increase costeffectiveness through a more targeted approach.

\subsection{Stress and Burnout in Teachers}

When an individual appraises an event as stressful, the body integrates multiple physiological systems to help cope with the stressor (Lazarus \& Folkman, 1984). One of these systems is the hypothalamic-pituitary-adrenal (HPA) axis, which helps to regulate levels of arousal in the body in response to homeostatic threats (Quigley, 2010; Tsigos \& Chrousos, 2002). The HPA axis system consists of the hypothalamus, the anterior pituitary gland, and the adrenal cortex, and helps to regulate homeostatic systems in the body (Tsigos \& Chrousos, 2002). As environmental stimuli are processed by the amygdala, they are sent through the central nucleus to the HPA axis and the sympathetic nervous system (SNS). When under stress, the SNS further accelerates activity in the hypothalamus, which controls the secretion of adrenocorticotropic hormone (ACTH) by the anterior pituitary gland. After ACTH binds to the receptors on the adrenal cortex, glucocorticoid hormones (i.e., cortisol in humans) are released into the blood stream (Ulrich-Lai \& Herman, 2009). Cortisol is utilized by the body to mobilize energy systems in order help the body deal with the stressor. While there are multiple ways to measure cortisol in the body, past research suggests that salivary cortisol is one of the most 
effective and practical endocrine markers of the human stress response (Scassellati, Bonvicini, Faraone, \& Gennarelli, 2012; Federenko, Nagamine, Hellhammer, Wadhwa, \& Wüst, 2004; Pruessner et al. 1997). Specifically, research indicates the Cortisol Awakening Response (CAR), a difference score between the measurement of cortisol immediately upon awakening and 30 minutes afterwards, is a useful and practical marker of allostatic load (Pruessner et al. 1997; Wust et al., 2000; Fries et al., 2009), and corresponds with other validated self-report measures of teacher stress and burnout (e.g., Pruessner, Hellhammer, \& Kirschbaum, 1999; Moya-Albiol, Serrano, \& Salvador, 2010). However, there is a discrepancy in the literature regarding the effects of stress and burnout on the CAR across different populations, with some evidence suggesting those in a caregiver role (similar to teachers) that experience higher amounts of social stress (as opposed to threats to the physical self) will tend to demonstrate a heightened CAR above the adaptive range (i.e., greater than $75 \%$ of waking cortisol levels; Miller, Chen, \& Zhou, 2007) while others suggest that the chronic effects of stress may ultimately lead to a blunted response (Pruessner et al., 1999; Thorn, Hucklebridge, Evans, \& Clow, 2006). However, more research is needed in order to better understand the relationship between measures of teacher stress and burnout with the CAR to determine the nature of this response in these contexts. Furthermore, funding agencies such as the National Institute of Health (NIH) have called for increased utilization of physiological measures and validated biomarkers (Insel et al., 2010), providing further support for the importance of assessing physiological measures of stress and burnout.

1.1.1 Teacher stress. Approximately one third of teachers report being stressed or extremely stressed (Borg \& Riding, 1991; Geving, 2007; Kyriacou \& Sutcliffe, 1979; 
Thomas, Clark, \& Lavery, 2003; Collie, Shapka, \& Perry, 2012). Research indicates teachers face a multitude of stressors in the school environment that each coincide with separate appraisal and coping responses (both physiological and psychological) which need to be engaged to meet the demands of the situation (Al-Fudail \& Mellar, 2008; De Dobile \& McCormick, 2005; Kyriacou, 2000, 2001; Travers \& Cooper, 1996; Dunham \& Varma, 1998; Huberman, 1993). For example, on a typical day, teachers will be exposed to a substantial number of social stressors (e.g., dealing with colleagues, administrators, and parents), time pressure (e.g., preparing lesson plans, grading, and adhering to curriculums for standardized testing), and other occupational demands specific to educating and managing students (e.g., teaching pupils who lack motivation and maintaining discipline in the classroom; Kyriacou, 2001) that each require a set of coordinated behaviors (Klusmann, Kunter, Trautwein, Lüdtke, \& Baumert, 2008) and the ability to flexibly shift attention throughout the day (Marzano, Marzano, \& Pickering, 2003) to effectively cope with each individual stressor. However, effectively managing stress requires several components of self-regulation, including significant attentional control, working memory capacity, and other executive functioning skills, which are often referred to as "higher order" skills given that they require elaborate networks and coordination amongst many different brain areas (Vohs \& Baumeister, 2016; McCarthy, \& Lambert, 2006; Boyle, Borg, Falzon, \& Baglioni, 1995). Research shows that effective stress management via acquisition of these types of skills can lead to decreases in teacher distress, increases in job satisfaction, and, subsequently, lower rates of teacher attrition (Neves de Jesus \& Conboy, 2001; Richardson \& Rothstein, 2008). 
Chronic stress has negative effects on teachers' regulation and coping abilities (i.e., stress management), in addition to their overall physical and psychological health (Schaufeli \& Greenglass, 2001). Hans Selye's (1956) popularized model of stress suggests that individuals move through the following three phases: Alarm, Resistance, and Exhaustion. A stressor is first perceived during the Alarm Stage, and the SNS is activated to prepare the body to cope with the threat. In the Resistance Stage, the body resists and compensates as the parasympathetic nervous system attempts to return physiological functioning back to normal, while the body focuses resources against the stressor and remains on alert. If the stressor or stressors continue beyond the body's capacity, the resources become exhausted and the body becomes more susceptible to disease; this is referred to as the Exhaustion Stage. If not addressed or dealt with effectively, this chronic stress has potential to overwhelm the body's capacity to manage the stress, which may lead to burnout syndrome (Schaufeli, Maslach, \& Marek, 1993) and a greater allostatic load (McEwen, 2004).

1.1.2 Teacher burnout. Burnout syndrome is defined as having the following three components: emotional exhaustion, depersonalization, and reduced personal accomplishment (Maslach \& Jackson, 1981; Maslach, Jackson, \& Leiter, 1996; Jennett, Harris, \& Mesibov, 2003; LeCompte \& Dworkin, 1991). Emotional exhaustion refers to a depletion of one's emotional resources (i.e., emotionally overextended, fatigued, loss of energy, wearing out, etc.). Although this component of burnout is typically the first to manifest behaviorally and the most frequently measured, the mere presence of symptoms of emotional exhaustion does not necessitate burnout syndrome (Maslach, Schaufeli, \& Leiter, 2001). The second component, depersonalization, refers to an individual feeling 
cynical, irritable, and having a negative attitude towards work. This is characterized by a lack of empathy or displaying detached interpersonal interactions. The final component is referred to as personal accomplishment, which is characterized by reduced self-efficacy and/or productivity. The nature of their profession often requires teachers to invest substantially in students, colleagues, and schools; yet, teachers are unlikely to feel as though they receive similar levels of reciprocal investment. Research shows that there are negative emotional, psychological, and professional repercussions when teachers' investments are not reciprocated (Van Horn, Schaufeli, \& Enzmann, 1999). The accumulation of this investment and associated negative outcomes resulting from a lack of reciprocal investment overtime predicts all three components of burnout, which functions as a compounding negative feedback loop that ultimately leads to burnout syndrome (Mearns \& Cain, 2003).

Although burnout is often described as a syndrome, it is typically conceptualized dimensionally based on number of symptoms present in each domain. Emotional exhaustion precedes other symptoms of burnout and is associated with, and conceptually caused by, any or all of the following factors: increased stress, interpersonal conflict, and lack of appropriate coping skills (Leiter, 1993). A lack of perceived social support often precludes symptoms of depersonalization (Greenglass et al., 1996) and research demonstrates that support from other teachers mitigates symptoms of burnout (Fiksenebaum \& Burke, 1996). The component of having a detached interpersonal style may further exacerbate symptoms of emotional exhaustion by isolating the individual from others (Fekete, 1991). This style often results in decreased personal accomplishment, propelled by feelings of emptiness, apathy, a mismatch between 
available and required personal resources to effectively manage the classroom, and, ultimately, a failure to apply appropriate coping mechanisms (Maslach et al., 2001; Fekete, 1991; Leiter, 1993). Teacher burnout negatively predicts both self-reported wellbeing and physiological indicators of health (Hakanen, Bakker, \& Schaufeli, 2006).

\subsection{Costs of Chronic Stress and Burnout}

Chronic stress and burnout have been shown to be associated with increased rates of a variety of mental and physical health problems, including the following: clinical depression, immune system functioning, obesity, cognitive aging, and multiple types of cancer (Zechmeister, Kilian, \& McDaid, 2008; Saleh \& Shapiro, 2008; Cohen, JanickiDeverts, \& Miller, 2007). A large body of research focuses on ways in which stress acts as a mechanism to increase rates of maladaptive behavioral patterns and coping strategies that are associated with the development of disease (e.g., smoking, sleep deprivation, poor adherence to medical regimen, and lack of physical exercise) and worsen over time through negative feedback loops (Cohen, Janicki-Deverts, \& Miller, 2007).

However, there is also literature suggesting that stress may lead to other diseases as a result of the degradation of physiological systems associated with the human stress response. This process is referred to as allostatic load (McEwen, 1998). The term "allostatic load" stems from the term "allostasis," which refers to a "maintenance of stability" or "remaining homeostatic through change" (McEwen, 1998). Chronic stress and symptoms of burnout are both associated with biomarkers of allostatic load (Juster, 2011). In reference to Selye's (1956) model of stress and adaptation, allostasis can be 
paralleled with the resistance phase of stress and juxtaposed with the exhaustion phase, the latter of which occurs as a result of overuse through repeated acute or chronic stress.

Above and beyond the societal cost of poor mental health, which is invariably linked with stress and accounts for an estimated 3-4\% of Gross Domestic Product (GDP) in developed nations such as the United States (Gabriel \& Liimatainen, 2000), the medical costs incurred by stress-induced chronic disease are estimated at over \$1 trillion annually (DeVol et al., 2007). Chronic stress has been associated with several debilitating and fatal diseases, including arthritis, diabetes, hypertension, and cardiovascular disease (Mattei, Demissie, Falcon, Ordovas, \& Tucker, 2010).

Specific to educational settings, teacher health problems can increase district health care and human resource costs associated with teacher illness, absenteeism, and attrition. With regard to students, teacher stress and burnout may also adversely affect student engagement and learning through teacher absenteeism, exhaustion, and diminished teaching effectiveness (Briner \& Dewberry, 2007; Jennings \& Greenberg, 2009). The ILO/UNESCO Joint Committee of Experts on the Application of the Recommendations Concerning the Status of Teachers (1994) reported that accumulated stress contributes significantly to teacher attrition, with the estimated cost of teacher dropout estimated at \$2.2 billion annually (Alliance for Excellent Education, 2004; 2005), representing a significant downstream cost of teacher stress and burnout. Given that approximately a third of teachers report being either stressed or "extremely stressed" (Borg \& Riding, 1991; Geving, 2007; Kyriacou \& Sutcliffe, 1979; Thomas, Clark, \& Lavery, 2003; Collie, Shapka, \& Perry, 2012), and up to 45\% of teachers experience burnout at some point during their careers (thus making teachers the largest vocational 
subgroup in the burnout literature; Schaufeli \& Enzmann, 1998), addressing teacher stress constitutes a major public health issue. Despite identified concerns and significant empirical investigation, it is still unclear how to effectively and efficiently combat teacher stress and burnout (Lambert \& McCarthy, 2006).

\subsection{Mindfulness: Theory and Intervention for Teachers}

Mindfulness originated from Eastern religious traditions and was reoriented as a psychological construct in the Western world (Kabat Zinn, 1990) where it was defined as "paying attention in a particular way: on purpose, in the present moment, and nonjudgmentally" (Kabat-Zinn, 1994, p. 4). Mindfulness skills represent a promising avenue in delivering "higher order" skills to teachers as a means of reducing stress and burnout (Ancona \& Mendelson, 2014). Modern theories of mindfulness (Renshaw, 2012; Brown, Ryan, \& Creswell, 2007; Shapiro et al., 2006) suggest that it consists of three primary tenets: Attentive Awareness ("the quality and duration of one's contact with whatever stimuli present themselves to one's mind in the here and now" [Renshaw \& O’Malley, 2014, p. 246]); Receptive Attitude (“one's outlook toward and reaction to particular stimuli that arise in awareness and are attended to in the present moment" [Renshaw \& O'Malley, 2014, p. 246]); and Intentionality, which has been conceptualized in two different ways. Specifically, some researchers (Renshaw \& O’Malley, 2014; Brown et al., 2007) have described intentionality as, "one's deliberate cultivation of an attentive awareness that is characterized by a receptive attitude, as opposed to simply recognizing or taking advantage of such features of one's mind whenever the chance occurs" [Renshaw \& O'Malley, 2014, p. 247]), alluding to the purposeful cultivation of one's attention. Others have described intentionality as the purpose for cultivating the 
mindful awareness, as Shapiro and colleagues (2006) note that, "When Western psychology attempted to extract the essence of mindfulness practice from its original religious/cultural roots, we lost, to some extent, the aspect of intention, which for Buddhism was enlightenment and compassion for all beings" (p. 375). This suggests that the reason for which an individual chooses to engage in a mindful practice implicitly impacts the qualitative nature of the practice. A study (Shapiro, 1992) exploring the intentions of meditation practitioners found that meditators' intentions shift along a continuum from self-regulation, to self-exploration, and finally, to self-liberation. The study also found that those whose goal was self-regulation and stress management attained more self-regulation, those whose goal was self-exploration attained greater selfexploration, and those whose goal was self-liberation moved toward self-liberation and compassionate service. These findings demonstrate functional implications for setting intention for the practice of mindfulness and justify its inclusion as a core conceptual component in developing MBIs. The current study utilizes a theoretical characterization of intentionality that encompasses both the former (i.e., the purposeful cultivation of mindfulness) and expands on this by including the elements of the latter conceptualization in emphasizing the importance of providing further direction for one's attention throughout one's practice (i.e., self-regulation and compassion). In line with past research, the current study conceptualized and measured mindfulness using the following five separate dimensions: Observe (i.e., the ability to recognize one's internal and external experience), describe (i.e., the ability to label experiences with words), act with awareness (i.e., the ability to maintain one's attention on actions in the present moment moment), non-reactivity (i.e., the ability to notice internal sensations such as 
thoughts and feelings without allowing one's attention to be pulled away by them), and nonjudgment (i.e., the ability to take a nonevaluative perspective on one's experiences).

MBIs have become increasingly popular in the Western world (Cullen, 2011) and there is now a substantial evidence base that demonstrates the effectiveness of MBIs for pain management, stress reduction, increased emotional regulation, decreased symptoms of depression and anxiety, and improvements in overall health and well-being (KabatZinn, 1982; Kirkwood, Rampes, Tuffrey, Richardson, \& Pilkington, 2005; Pilkington, Kirkwood, Rampes, \& Richardson, 2005; Carmody \& Baer, 2008; Grossman, Niemann, Schmidt, \& Walach, 2004). Results from MBIs within occupational settings also indicate significant reductions in stress and increases in well-being (Escuriex \& Labbê, 2011; Irving, Dobkin, \& Park, 2009; Virgili, 2013). One mechanism that has been posited to account for significant portion of variance in the effectiveness of MBIs is an increased capacity to down-regulate bottom-up, fast-onset stress reactions and to up-regulate slow, top-down nondominant response tendencies (Miyake, Friedman, Emerson, Witzki, \& Howerter, 2000; Roeser et al., 2013). This process is most closely associated with three of five dimensions (i.e., observe, describe, and act with awareness) that are included on validated measures of mindfulness (Baer et al., 2008). These processes allow individuals to better "recognize and regulate" (Roeser et al., 2013, p. 3) reactions to stressors in the environment and manage stress more effectively.

The application of mindfulness in teaching as a means of reducing stress and promoting well-being has become a popular endeavor over the past ten years (Hwang, Bartlett, Greben, \& Hand, 2017). There is substantial variation in the content covered among various MBIs for teachers (e.g., Ancona \& Mendelson, 2014; Roeser et al., 2012). 
Many MBIs are characterized by either meditation or physical yoga practice (asana; Greenberg \& Harris, 2012), with most mindfulness training programs for teachers focusing on the former in various capacities via training the mind through focusing one's attention in a chosen manner (e.g., "practices vary and include attending to the breath or body sensations, eating with awareness, open awareness of experience, and cultivation of loving kindness" [Ancona \& Mendelson, 2014, p. 157]). These meditative practices aim to promote increased cognitive and emotional capacity via stimulation of the prefrontal cortex and other relevant brain regions (Kilpatrick et al., 2011; Lutz, Slagter, Dunne, \& Davidson, 2008). Additionally, some MBIs for teachers focus on emotion skills instruction, mindful awareness practices, and compassion building activities to provide teachers with skills to reduce their emotional stress and give them tools to build more effective relationships with their students (e.g., Jennings \& Greenberg, 2009; Harris, Jennings, Katz, Abenavoli, \& Greenberg, 2016). Although some of the existing literature suggests that MBIs have the potential to reduce teachers' stress, burnout, and other psychological symptoms (e.g., depression, anxiety, etc.; Franco, Mañas, Cangas, Moreno, \& Gallego, 2010; Flook et al., 2013; Gold et al., 2010; Jennings, Frank, Snowberg, Coccia, \& Greenberg, 2013; Roeser et al., 2013), there have been mixed findings across studies (Reiser et al., 2016; Reiser \& McCarthy, 2018; Jennings, Snowberg, Coccia, \& Greenberg, 2011; Frank, Reibel, Broderick, Cantrell, \& Metz, 2015; Klingbeil \& Renshaw, 2018) that may be accounted for by discrepancies in the methodological rigor and variation in intervention dosage (i.e., the number of total direct contact hours). 


\subsection{Methodological Rigor}

The standards of methodological rigor first developed by the Task Force for Promotion and Dissemination of Psychological Procedures Assessment (American Psychological Association, 1995), and later adjusted by Chambless and Hollon (1998), established guidelines for determining the degree to which studies seeking to identify empirically-validated interventions made a cogent contribution to the literature. The majority of MBI studies to reduce teacher stress and burnout (see table 1.1 for overview of studies; Ancona \& Mendleson, 2014; Benn et al., 2012; Beshai et al., 2016; Flook et al., 2013; Frank et al., 2015; Reiser \& McCarthy, 2018; Gold et al., 2010; Jennings et al., 2011; Reiser et al., 2016) are missing multiple elements of methodological rigor, including a lack of a control group (Gold et al., 2010; Jennings et al., 2011), failure to randomize to condition when a control group was included (Beshai et al., 2016; Reiser et al., 2016), and the utilization of small sample sizes (i.e., less than 25 participants per group; Anocona \& Mendleson, 2014; Benn et al., 2012; Flook et al., 2013; Frank et al., 2015; Gold et al., 2010; Reiser et al., 2016; Reiser \& McCarthy, 2018). Among existing MBI studies for teachers, only three can be considered "gold standards," meeting all six criteria proposed by these guidelines (see table 1.2; Harris et al, 2016; Jennings et al., 2013; Roeser et al., 2013) and only two of these (Harris et al., 2016; Roeser et al., 2013), and one other preliminary study that only included a sample size of 18 teachers (Flook et al., 2013), also included physiological measures of stress and burnout despite major funding agencies such as NIH calling for their increased use to improve methodological rigor across the field of psychology (Insel et al., 2010). 
Despite lacking elements of methodological rigor, many of these more "preliminary" studies are useful for generating hypotheses regarding the minimally effective dose of MBIs, as there is substantial variability in dosage across these studies, which differs from the more rigorous studies (Harris et al., 2016; Jennings et al., 2013; Roeser et al., 2013) that are generally longer and have less variability. The current study seeks to expand on this literature by addressing some of these gaps in the methodological rigor of previous studies (i.e., inclusion of a waitlist-control group and randomization to groups), by utilizing physiological measures of stress and burnout, and by assessing a dosage that is lower than the majority of MBI studies.

\subsection{Efficacy of Mindfulness-Based Interventions for Teachers}

Several MBI studies (Jennings et al., 2013; Ancona \& Mendelson, 2014; Benn et al. 2012; Gold et al., 2010; Jennings et al., 2011; Reiser et al., 2016) have demonstrated small-to-medium reductions of teacher's self-reported stress. Additionally, one study (Beshai et al., 2016) lacking a few key elements of methodological rigor (i.e., randomization to groups, utilization of physiological measures of stress) demonstrated the largest effect size $(d=1.23)$ of any MBI study for teacher stress. In contrast, only a few studies (Flook et al., 2013; Harris et al., 2016; Roeser et al., 2013; Ancona \& Mendleson, 2014; Frank et al., 2015; Jennings et al., 2013) have examined teacher burnout; only two (Flook et al., 2013; Roeser et al., 2013) of these studies demonstrated a significant effect. Given the chronic and inexorable stressors faced by teachers (Kyriacou, 2001; Smith et al., 2000), and the substantial costs associated with teacher burnout (McEwen, 1998; Schaufeli \& Enzmann, 1998), it is important for MBIs to examine this construct. Being that few studies demonstrated significant reductions in 
burnout, it is also important to identify the mechanisms which account for this change to inform future intervention development.

Although Lazarus and Folkman (1984) emphasize the importance of appraisal in the human stress response, not all stress is psychologically mediated (Cohen, Kessler, \& Gordon, 1997). For example, Selye $(1956 ; 1974)$ posits that an individual's physiological stress response (i.e., alarm, resistance, and exhaustion) follows a similar pattern regardless of the appraisal, but not all individuals may appraise particular events as stressful since some may have greater self-efficacy regarding their ability to cope with these demands (Bandura, 1991; 2003; 2005; Betoret, 2006). Furthermore, McEwen $(1998 ; 2004)$ has demonstrated that chronic stress can cause alterations to these physiological systems overtime. Therefore, although self-reported changes to stress and burnout are useful preliminary indicators that MBIs can reduce teacher stress and burnout, it is imperative to also utilize physiological measures of stress, as they can capture elements of stress and burnout that self-reported measures cannot. Of the physiological measures of stress, CAR is one of the most effective and practical for capturing chronic stress (Chida \& Steptoe, 2009; Scassellati, Bonvicini, Faraone, \& Gennarelli, 2012; Federenko, Nagamine, Hellhammer, Wadhwa, \& Wüst, 2004; Pruessner et al. 1997) making it ideal for use across MBI studies for teachers. Literature suggests there is corroboration between physiological measures of stress, particularly CAR, and teacher stress and burnout (Pruessner et al., 1999), but there are mixed findings in the three existing MBI studies for teachers that utilized CAR (Flook et al., 2013; Harris et al., 2016; Roeser et al., 2013). Among the two gold-standard studies that measured CAR, one study (Harris et al., 2016) found a significant reduction in CAR (i.e., a blunted 
response) for the control group $(d=.64)$, whereas the other (Roeser et al., 2013) found a small but non-statistically significant reduction in the intervention group $(d=.22)$. Only the latter of these studies (i.e., Roeser et al., 2013) corroborated findings of physiological markers of stress with self-reported stress and burnout (see table 1.1 for details), which further supports the need for utilizing both of these different measurement modalities.

The other study that measured CAR (Flook et al., 2013) measured only one postwaking time point in their study (i.e., 30 minutes post-waking) to reduce measurement burden on teachers and identified a nearly identical reduction in cortisol secretion at this time $(3.13 \mathrm{nmol} / \mathrm{L}$ to $3.06 \mathrm{nmol} / \mathrm{L})$; however, there was a significant flattening of the cortisol response following the intervention for the control group in comparison to the intervention group $(3.30 \mathrm{nmol} / \mathrm{L}$ to $2.67 \mathrm{nmol} / \mathrm{L} ; d=.70)$. This preliminary study also found a significant reduction in two symptom domains of burnout (Emotional Exhaustion and Personal Accomplishment) for the intervention group, concomitant with an increase in symptoms of burnout on the Personal Accomplishment subscale for the control group. The researchers hypothesized that these findings indicated a physiological profile of burnout syndrome in teachers as previous research has suggested that a dampened response is likely to occur in those that are experiencing symptoms of burnout, hypothetically resulting from a failure to adaptively manage chronic stress (Dedovic \& Ngiam, 2015; Wardenaar et al., 2011). Previous literature shows this pattern is particularly salient for those in caretaking roles who have been shown to manage their stress more adaptively during periods of heightened physiological stress (Adam \& Gunnar, 2001). However, there are also studies (De Vente, Olff, Van Amsterdam, Kamphuis, \& Emmelkamp, 2003; Grossi, Perski, Ekstedt, Johansson, Lindström, \& 
Holm, 2005) that show there is an increased CAR in those experiencing burnout as opposed to a dampened response, suggesting that corroborating physiological data with self-report can help to elucidate these relations and enhance the significance of findings in MBI studies for teachers.

Despite the unique contributions of physiological measures of stress (Winters, 2012; Fred, Rowland, \& Ferris, 1984) as a means of better understanding the effects of MBIs on teacher stress and burnout, as well as a call for increased use of validated biomarkers from many funding agencies (Insel et al., 2010), there is still a need for more MBI studies for teachers to utilize physiological markers of stress. The current study contributes to this burgeoning literature by assessing the changes in CAR over the course of an academic semester in a group of teachers receiving a bMBI and a control group. Furthermore, the current study utilized the lowest dosage of any MBI to measure CAR, which contributes to the understanding of a minimally effective dose in the literature regarding physiological markers of stress.

\subsection{Dosage}

Reviews of the extant literature (Emerson et al., 2017; Hwang et al., 2017; Klingbeil \& Renshaw, 2018; Lomas, Medina, Ivtzan, Rupprecht, \& Eiroa-Orosa, 2017) have highlighted the significant need to identify minimally effective dose to maximize cost-effectiveness, with intervention dose highly varied across previous studies. Ideally, the minimally effective dose can be identified as the intervention that required the least exposure and still produced a noticeable and meaningful effect. Unfortunately, there was little variability in the dosages (range $=21-30$ hours; $\bar{x}=25.67$ hours; $\tilde{x}=24$ hours) 
amongst the three studies (Flook et al., 2013; Harris et al., 2016; Roeser et al., 2013) that collected physiological measures of stress in comparison to the other MBI studies (range $=4.5-36$ hours; $\overline{\mathrm{x}}=18.5$ hours; $\tilde{\mathrm{x}}=16$ hours) making it difficult to identify a minimally effective dose. Of the studies that collected data on CAR, the shortest study (i.e., 21 hours; Harris et al., 2016) was the only one that demonstrated a significant change in CAR. The contrast in dosage between Harris and colleagues' (2016) study and the other two studies (26 hours and 30 hours, respectively; Flook et al., 2013; Roeser et al., 2013) supports findings from a recent meta-analysis (Klingbeil \& Renshaw, 2018) that there are diminishing returns, and potentially iatrogenic effects, for MBIs with higher dosages. The meta-analysis, which included all existing MBI studies for teachers (including those published in other languages and others that were not published in peer-reviewed journals, such as dissertations), indicated that iatrogenic effects occur after approximately 24 direct contact hours. Although this finding appears to provide some insight into the upper end of effective doses, findings related to self-reported stress and burnout in some less rigorous preliminary studies suggest the minimally effective dose may be substantially lower than that.

For example, Beshai and colleagues' (2016) demonstrated the largest effect size $(d=1.23)$ of any study in reducing self-reported stress despite including only 11 contact hours. Similarly, Ancona \& Mendleson's (2014) study also demonstrated small-tomedium effect sizes for teacher stress $(d=.54)$ and burnout $(d=.42)$ that were comparable to more methodologically rigorous studies despite including only 4.5 contact hours. These findings suggest that the maximally-effective dose likely falls between 11 and 24 hours, while the minimally effective dose may include as few as 4.5 direct contact 
hours. The findings from these preliminary studies, in aggregate with those from more methodologically rigorous studies, suggest a need for further exploration of a minimally effective dose to reduce teacher stress and burnout that includes physiological measurement of these constructs in a MBI that includes between 4.5 and 11 direct contact hours.

1.6.1 Mechanisms of Change. Identifying key mechanisms of change is also critical for informing what key components are needed to support the effectiveness and efficiency of future MBIs for teachers. However, of the previous MBI studies to reduce teacher stress and burnout, only one (Roeser et al., 2013) measured the impact of changes in mindfulness on these outcomes, and only a few (Flook et al., 2013; Frank et al., 2015; Gold et al., 2010; Harris et al., 2016; Jennings et al., 2011; Jennings et al., 2013) measured the separate dimensions of mindfulness at all. Furthermore, none of these studies fell in the recommended dosage range of 4.5-11 hours, but differences amongst the four studies within that range (Ancona \& Mendleson, 2014; Beshai et al., 2016; Reiser et al., 2016; Reiser \& McCarthy, 2018) provided insight into how these proposed mechanisms of change (i.e., mindfulness skills) may function based on the differences in the components of these interventions that aimed to reduce teacher stress and burnout .

Among the studies falling in the recommended dosage range, the two studies that demonstrated a significant effect on teacher stress (Ancona \& Mendleson, 2014; Beshai et al., 2016) differed substantially in curriculum content and conceptual framework compared to the two studies that did not demonstrate significant effects (Reiser et al., 2016; Reiser \& McCarthy, 2018). The two studies that did not demonstrate significant effects (Reiser et al,, 2016; Reiser \& McCarthy, 2018) employed a derivation of the 
SPAM program, which emphasized social support and psychoeducation about mindfulness, whereas the former studies that did demonstrate significant effects (Beshai et al., 2016; Ancona \& Mendleson, 2014) were similar to the more methodologically rigorous studies derived from the CARE program (Jennings et al., 2013) and MBSR training protocols (Flook et al., 2013; Harris et al., 2016; Roeser et al., 2013), which both emphasize cultivating mindfulness to help effectively self-regulate stress in the classroom. There were also mixed findings regarding statistical changes in general mindfulness skills amongst these lower dosage studies. One of the studies using an MBSR approach that focused on self-regulation (Ancona \& Mendleson, 2014) did not utilize a measure of mindfulness skills, and one study utilizing the SPAM program (Resier et al., 2016) did not demonstrate significant changes for mindfulness. However, both Beshai and colleagues (2016; utilizing an MBSR approach) and Reiser and McCarthy (2018; utilizing a derivation of the SPAM program) demonstrated significant increases in mindfulness skills despite only the former (Beshai et al., 2016) demonstrating significant reductions in teacher stress, which suggests that not all improvements in general mindfulness lead to reductions in teacher stress and burnout. As none of these studies analyzed the separate dimensions of mindfulness (i.e., observing, describing, acting with awareness, non-judgment, and non-reactivity), it is difficult to know which of the separate dimensions of mindfulness may account for changes in teacher stress and burnout in these lower dosage studies. Moreover, the contrast of findings in these studies may suggest that the differences in intervention components among the studies (i.e., practicing mindfulness skills and emphasizing its utility to selfregulation as opposed to emphasizing psychoeducation about mindfulness and social 
support) led them to target different facets of mindfulness that differentially impact changes in stress and burnout for teachers.

The more rigorous studies of higher dosages that measured all of these separate facets of mindfulness (Jennings et al., 2013; Flook et al., 2013; Harris et al., 2016) demonstrated significant changes on only three of these dimensions (i.e., observing, describing, and non-reactivity), but these studies did not measure the effects of changes in mindfulness skills on teacher stress and burnout making it difficult to know the extent to which these components of mindfulness acted as mechanisms of change. Although Roeser and colleagues (2013) demonstrated that general mindfulness skills predicted reductions in teacher stress and burnout, this study did not analyze the five separate dimensions of mindfulness. Therefore, a gap exists in the literature as no study to date has measured the effects of changes to the separate dimensions of mindfulness on teacher stress and burnout.

Given none of the studies in the identified minimally effective dosage range analyzed the effect of the intervention on the five separate dimensions of mindfulness, the current study will contribute to the literature by being the first in this range to do so. Furthermore, no MBI studies have assessed the impact of changes to these dimensions of mindfulness on teacher stress and burnout; yet, previous research in non-clinical samples suggests the effects of improvements in these different dimensions varies for differing outcomes (e.g., cardiovascular health vs negative affect; Prazak et al., 2012). Therefore, it is important to assess the effects of these separate dimensions on teacher stress and burnout in order to more effectively target the mechanisms that produce change in these domains. As such, the current investigation analyzed the degree to which these separate 
dimensions of mindfulness functioned as mechanisms of change on self-reported and physiological measures of teacher stress and burnout. The findings from these analyses, integrated with findings regarding the efficacy of a bMBI falling within the identified range (i.e., 4.5-11 hours) can further inform the literature regarding the effective components to target in MBIs that seek to optimize their utilization of resources.

\subsection{The Current Study}

Previous studies investigating MBIs for teachers identified generally positive outcomes; however, many of these studies enacted burdensome training models that demonstrated diminishing returns and, in some instances, iatrogenic effects (Klingbeil \& Renshaw, 2018). The lack of methodologically rigorous research and absence of physiological measures of stress in studies of shorter duration make the identification of a minimally effective dose more difficult. Furthermore, there is ambiguity regarding which specific domains of mindfulness account for change in these therapeutic outcomes, which would allow for refinement of intervention protocols to be used for studies seeking to optimize the effects of MBIs. To address these gaps in research, the current study designed and implemented a brief ( 4 sessions; 6 direct contact hours) MBI (bMBI), utilized a rigorous randomized controlled research design, included assessment of physiological measures of stress, and explored the impact of specific domains of mindfulness. Together, the findings of the current study will provide insight into the minimally effective dose of MBIs for teachers. The primary aims of the study are addressed through the following research questions below. 
Firstly, is the bMBI effective in decreasing stress (self-report and CAR), burnout (self-report), and general psychological distress (self-report) by 1) decreases in the number of teachers presenting with a maladaptive CAR from pre- to post-intervention, 2) reductions in self-reported perceived stress and burnout (measured by the Teacher Stress Inventory and Maslach Burnout Inventory, respectively) from pre- to post- intervention, and 3) reductions in self-reported psychological symptoms (measured by the General Symptom Index and Anxiety, and Depression Subscales of the Symptom Assessment-45) from pre- to post-intervention? We hypothesized that there would be significant improvements for all measures of stress, burnout, and psychological distress for teachers receiving the bMBI but no significant changes in the waitlist-control group.

Secondly, is the intervention effective in improving mindfulness skills and its five dimensions (i.e., observe, describe, act with awareness, nonjudgment, and non-reactivity) as measured by increases in self-reported mindfulness (measured by the Five-Facet Mindfulness Questionnaire; FFMQ)? Additionally, for those who received the intervention, do the changes from pre- to post-intervention in the separate dimensions of mindfulness differentially impact teacher outcomes (i.e., teacher stress, burnout, and general psychological symptoms). We hypothesized that teachers receiving the bMBI would demonstrate a significant increase on the full scale and each of the five separate subscales of the FFMQ from pre- to post-intervention, but there would be no significant changes in the waitlist-control group. Furthermore, we expected that our novel investigation of the relation between changes in mindfulness and its separate dimensions with teacher outcomes would demonstrate differences in the extent to which components of mindfulness functioned as mechanisms of change for the various teacher outcomes. 
Through identifying key mechanisms needed for facilitating positive change in targeted outcomes, this aim further supports the overarching goal of optimizing intervention effectiveness with minimal resource and time demands. 
Table 1.1. Details of previous MBI studies for teachers.

\begin{tabular}{|c|c|c|c|c|c|c|}
\hline $\begin{array}{l}\text { Author } \\
\text { (year) }\end{array}$ & $n$ & $\begin{array}{c}\text { Research } \\
\text { Design/School } \\
\text { Level }\end{array}$ & Dose & $\begin{array}{l}\text { Primary } \\
\text { Outcomes } \\
\text { for Stress } \\
\text { and Burnout }\end{array}$ & $\begin{array}{c}\text { Mindfulness } \\
\text { Skills } \\
\text { Outcomes }\end{array}$ & $\begin{array}{c}\text { Other } \\
\text { Comments } \\
\text { Related to } \\
\text { Proposed } \\
\text { Model }\end{array}$ \\
\hline $\begin{array}{l}\text { Ancona } \\
\& \\
\text { Mendels } \\
\text { on } \\
(2014)\end{array}$ & 43 & RCT/Various & $\begin{array}{l}6 \\
\text { sessions; } \\
4.5 \text { total } \\
\text { contact } \\
\text { hours }\end{array}$ & $\begin{array}{l}\text { Teacher } \\
\text { Stress } \\
\text { Inventory } \\
\text { (change } \\
\text { comparisons } \\
\text { ): } \mathrm{p}<.10 ; \mathrm{d} \\
=.54 \\
\text { MBI-ES EE } \\
\text { (change } \\
\text { comparisons } \\
\text { ): n.s.; d = } \\
.42\end{array}$ & $\begin{array}{l}\text { Qualitative } \\
\text { reports of } \\
\text { increased } \\
\text { mindfulness } \\
\text { skills }\end{array}$ & $\begin{array}{l}\text { Qualitative } \\
\text { reports of } \\
\text { increased self- } \\
\text { efficacy for } \\
\text { self-regulation } \\
\text { of stress } \\
\text { management }\end{array}$ \\
\hline $\begin{array}{l}\text { Benn et } \\
\text { al. } \\
(2012)\end{array}$ & 38 & RCT/Mixed & $\begin{array}{l}11 \\
\text { sessions; } \\
36 \text { total } \\
\text { contact } \\
\text { hours }\end{array}$ & $\begin{array}{l}\text { PSS (change } \\
\text { comparisons } \\
\text { ): } \mathrm{p}<.10 ; \mathrm{d} \\
=.40\end{array}$ & $\begin{array}{l}\text { FFMQ total } \\
\text { (change } \\
\text { comparisons } \\
\text { ): } \mathrm{p}<.05 ; \mathrm{d} \\
=.52\end{array}$ & $\begin{array}{l}\text { Emotion } \\
\text { Regulation at } \\
\text { Work Self- } \\
\text { Efficacy Scale } \\
\text { (change } \\
\text { comparisons): } \\
\mathrm{p}<.10 \text {; d = } \\
.55\end{array}$ \\
\hline $\begin{array}{l}\text { Beshai et } \\
\text { al. } \\
(2016)\end{array}$ & 89 & $\begin{array}{l}\text { Non- } \\
\text { Randomized } \\
\text { Control } \\
\text { Trial/Seconda } \\
\text { ry }\end{array}$ & $\begin{array}{l}9 \\
\text { sessions; } \\
11 \text { total } \\
\text { hours }\end{array}$ & $\begin{array}{l}\text { PSS (change } \\
\text { comparisons } \\
\text { ): } \mathrm{p}<.05 ; \mathrm{d} \\
=1.23\end{array}$ & $\begin{array}{l}\text { FFMQ total } \\
\text { (change } \\
\text { comparisons } \\
\text { ): } \mathrm{p}<.05 ; \\
1.45\end{array}$ & N/A \\
\hline $\begin{array}{l}\text { Flook et } \\
\text { al. } \\
(2013)\end{array}$ & 18 & $\begin{array}{l}\text { RCT/Element } \\
\text { ary }\end{array}$ & $\begin{array}{l}9 \\
\text { sessions; } \\
26 \text { total } \\
\text { contact } \\
\text { hours }\end{array}$ & $\begin{array}{l}\begin{array}{l}\text { Reduction } \\
\text { (pre-post) }\end{array} \\
\text { for } \\
\text { intervention } \\
\text { group on } \\
\text { MBI-ES EE } \\
\text { and MBI-ES } \\
\text { PA (p<.05) } \\
\text { and Increase } \\
\text { for control } \\
\text { group on } \\
\text { MBI-ES PA } \\
(p<.10) ;\end{array}$ & $\begin{array}{l}\text { Increase in } \\
\text { FFMQ } \\
\text { Observe (p } \\
<.05 \text { ) and } \\
\text { Describe (p } \\
<. .01 \text { ) } \\
\text { Subscales } \\
\text { for } \\
\text { Intervention } \\
\text { Group; } \\
\text { FFMQ (post } \\
\text { comparisons } \\
\text { ): Observe: }\end{array}$ & $\begin{array}{l}\text { Improvements } \\
\text { in Sustained } \\
\text { Attention and } \\
\text { AGN Tot } \\
\text { Com Task (p } \\
<.05 \text { ) for } \\
\text { Intervention } \\
\text { Group; } \\
\text { Sustained } \\
\text { Attention } \\
\text { (post } \\
\text { comparisons): } \\
d=-.03 \text {; AGN }\end{array}$ \\
\hline
\end{tabular}


Frank et

al.

(2015)

Gold et

al.

(2010)

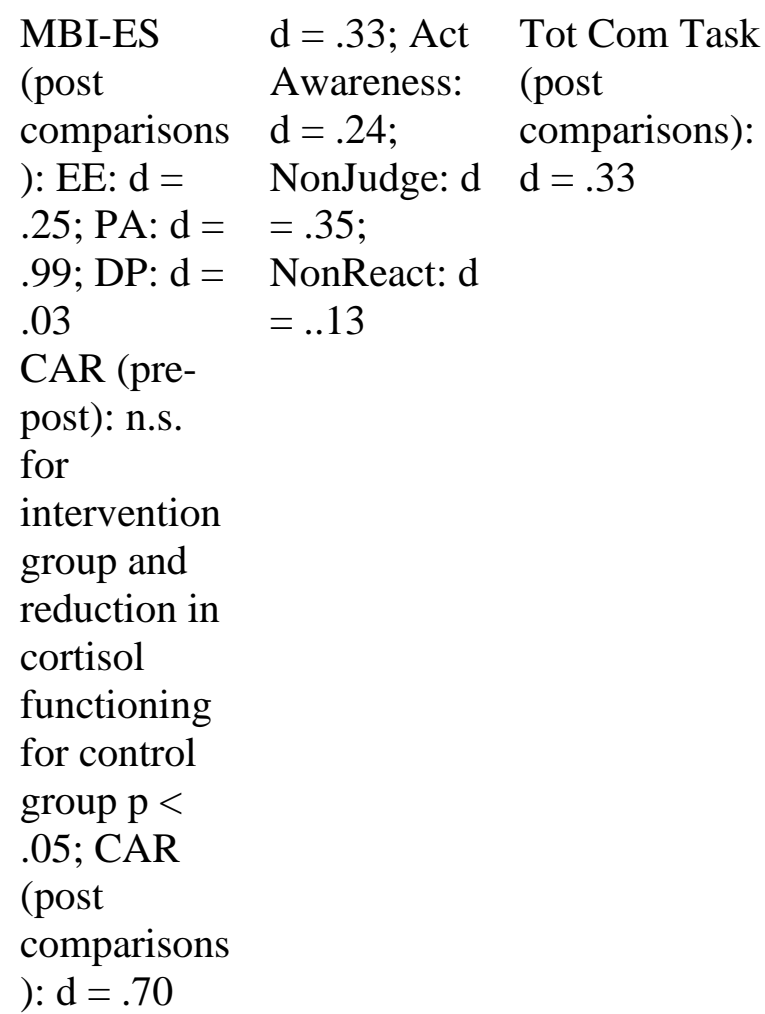

FFMQ ASRES

(change (change

comparisons comparisons):

): Observe: Acknowledg

MBI-ES $\quad \mathrm{p}<.05 ; \mathrm{d}=$ ment: $\mathrm{p}<.05$;

$1.85 ; \quad \mathrm{d}=1.25$;

Describe: $\mathrm{p}$ Calmness: $\mathrm{p}<$ $<.10 ; \mathrm{d}=\quad .05 ; \mathrm{d}=1.47$;

.74; Act Present

Awareness: Moment: $\mathrm{p}<$ n.s.; $\mathrm{d}=.09$; DP: n.s.; $d=$ $-.33$

$\mathrm{p}<.05 ; \mathrm{d}=.05 ; \mathrm{d}=1.25$

1.06;

Acceptance:

NonJudge: $\mathrm{p}$ n.s.; $\mathrm{d}=.37$

$<.05 ; \mathrm{d}=$

1.50

NonReact; $p$

$<.05 ; \mathrm{d}=$

1.58

9

Pre-Post
Comparison
Trial/Element
ary

sessions;

25 total

contact

hours
DASS

Stress (pre-

post): $\mathrm{p}<$ $.05 ; \mathrm{d}=.70$
Increase in

KIMS

Accept

without N/A 


\section{(pre-post; p \\ $<.05)$; No \\ significant \\ change in \\ other 3 \\ subscales of \\ KIMS}

PSS (post

comparisons

): n.s.; $d=$

.41; TUS: $p$

$<.10 ; \mathrm{d}=$

.43; MBI-

ES: EE:

n.s.; $d=.25$;

PA: n.s. $d=$

.23; DP: $\mathrm{p}<$

$.10 ; \mathrm{d}=.48$

64

Harris et

al.

(2016)

Jennings

et al.

(2011;

Study 1

only)
$64 \mathrm{RCT} /$ Seconda ry
Non-

Randomized Control Trial /Elementary sessions; 21 total contact hours
CAR: $\mathrm{p}<$

$.05 ; \mathrm{d}=.64$

(i.e., a

blunted

response for

control

group); Cort

AUC: n.s.; d

$=.16$;

Systolic BP:

n.s.; $d=.39$;

Diastolic

BP: $\mathrm{p}<.05$;

$\mathrm{d}=.52$

TUS (prepost): TUS

Task-

4

sessions;

30 total

contact

hours
Hurry: $\mathrm{p}<$
Related .05.; $\mathrm{d}=$ .24; TUS

General

Hurry: $\mathrm{p}<$ $.10 ; d=.27$
TSES (post comparisons):

Classroom

Engagement:

$\mathrm{p}<.05 ; \mathrm{d}=$ .54

FFMQ (post

comparisons

): Observe:

$\mathrm{p}<.05 ; \mathrm{d}=$ .56 ;

Describe:

n.s.; $d=.14$;

Act

Awareness:

n.s.; $d=.26$;

NonJudge:

n.s.; $d=.41$;

NonReact;

n.s.; $d=-.07$ 
Jennings

et al.

(2013)

Reiser et

al.

(2016)

\section{RCT/Mixed}

4 sessions; TUS

30 total General

contact

hours $\mathrm{d}=.32$;
$<.10 . ; \mathrm{d}=$

.39;

NonReact; $\mathrm{p}$

$<.05$. $\mathrm{d}=$

.78; IMT: $\mathrm{p}$

$<.05 ; \mathrm{d}=$

.48

FFMQ (post TSES (post

comparisons comparisons):

): Total: $\mathrm{p}<$ Classroom

$.05 ; \mathrm{d}=.57$; Management:

Task-

Related

Hurry: n.s.;

Observe: $\mathrm{p}$

$<.05 ; \mathrm{d}=$ .69 ; n.s.; $d=.31$

ERQ (post

comparisons):

Describe: Reappraisal: $\mathrm{p}$

n.s.; $\mathrm{d}=\quad<.05 ; \mathrm{d}=.80$;

.156; Act Suppression:

Awareness: $\quad \mathrm{p}<.10 ; \mathrm{d}=$

n.s.; $d=.13 ; \quad .43$

EE: n.s.; $\mathrm{d}=$

.04; DP:

n.s.; $d=.06$;

PA: $\mathrm{p}<.10$

$\mathrm{d}=.40$

NonJudge:

n.s.; $d=.12$;

NonReact; $p$

$<.05$; $\mathrm{d}=$ .73

Classroom

Appraisal of

6 Resources No

sessions; and significant

6 total Demands changes on

contact (post- the FFMQ-

hours comparisons SF

): n.s.; d =

.23

\section{Classroom}

Appraisal of

Reiser \&

McCarth

y (2018)
15 specify
N/A
Resources

and

Demands

(change

comparisons

at post):

n.s.; $d=.03$
Qualitative reports of increases in self-efficacy to selfregulate stress management skills 


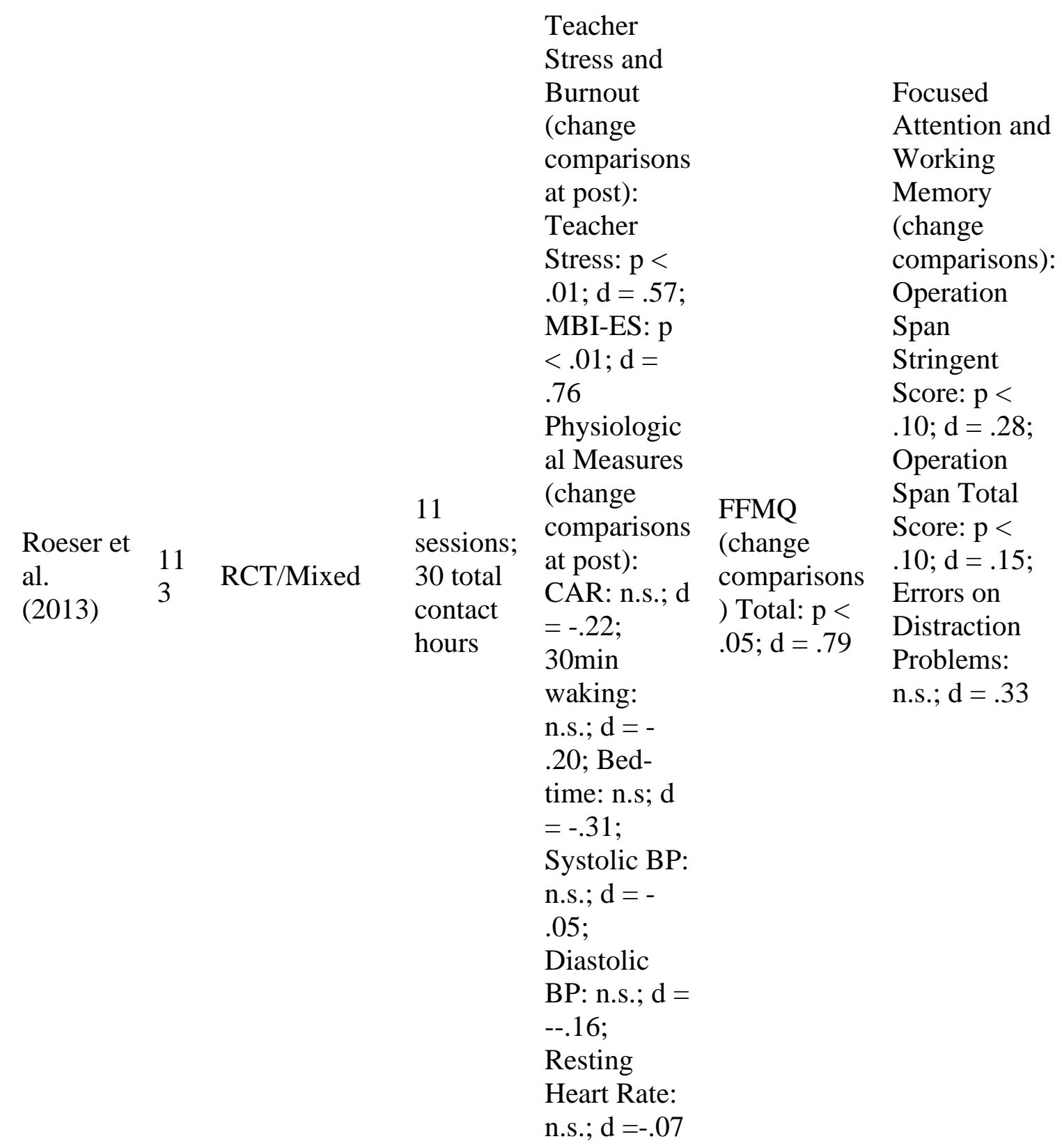

Notes: PSS - Perceived Stress Scale; MBI-ES - Maslach Burnout Inventory- Educators Survey; EE - Emotional Exhaustion subscale of Maslach Burnout Inventory; DP Depersonalization subscale of Maslach Burnout Inventory; PA - Personal Accomplishment subscale of Maslach Burnout Inventory; DASS - Depression Anxiety Stress Scales; TUS - Time Urgency Scale; CAR - Cortisol Awakening Response; Cort AUC - Cortisol Response Area Under the Curve; BP - Blood Pressure; FFMQ - Five Factor Mindfulness Questionnaire; FFMQ-SF - Five Factor Mindfulness Questionnaire Short Form; KIMS - Kentucky Inventory of Mindfulness Skills; IMT - Interpersonal Mindfulness in Teaching Inventory; PALS - Positive Adaptive Learning Scales; AGN 
Tot Com Task - Affective Go-No Go task Total Commissions; ASRES - Affective SelfRegulation Efficacy Scale; TSES - Teaching Self-Efficacy Questionnaire; ERQ Emotion Regulation Questionnaire Positive Cohen 's d values show a benefit towards the intervention over control or a reduction in symptom from pre-post where applicable. 
Table 1.2. Methodological rigor of reviewed studies.

\begin{tabular}{|c|c|c|c|c|c|c|c|c|}
\hline $\begin{array}{c}\text { Author } \\
\text { (year) }\end{array}$ & $\begin{array}{l}\text { Defined } \\
\text { Problem } \\
\text { and } \\
\text { Population }\end{array}$ & $\begin{array}{l}\text { Rand } \\
\text { omiz } \\
\text { ed }\end{array}$ & $\begin{array}{c}25+ \\
\text { Participants } \\
\text { per Group }\end{array}$ & $\begin{array}{l}\text { Comp } \\
\text { arative } \\
\text { Treat } \\
\text { ment }\end{array}$ & $\begin{array}{c}\text { Treatment } \\
\text { Manual/ } \\
\text { Curriculum }\end{array}$ & $\begin{array}{c}\text { Validated } \\
\text { / } \\
\text { Reliable } \\
\text { Outcome } \\
\text { Measures }\end{array}$ & $\begin{array}{c}\text { Physiological } \\
\text { Stress/ } \\
\text { Burnout } \\
\text { Measure }\end{array}$ & $\begin{array}{l}\text { Total } \\
\text { Score }\end{array}$ \\
\hline Ancona \& & & & & & & & & \\
\hline $\begin{array}{l}\text { Mendelson } \\
\text { (2014) }\end{array}$ & $\mathrm{x}$ & $\mathrm{x}$ & & $\mathrm{x}$ & $\mathrm{x}$ & $\mathrm{x}$ & & 5 \\
\hline $\begin{array}{l}\text { Benn et al. } \\
\text { (2012) }\end{array}$ & $\mathrm{X}$ & $\mathrm{X}$ & & $\mathrm{x}$ & $\mathrm{x}$ & $\mathrm{X}$ & & 5 \\
\hline $\begin{array}{l}\text { Beshai et } \\
\text { al. (2016) }\end{array}$ & $\mathrm{x}$ & & $\mathrm{X}$ & $\mathrm{x}$ & $\mathrm{x}$ & $\mathrm{X}$ & & 5 \\
\hline $\begin{array}{l}\text { Flook et al. } \\
\quad(2013)\end{array}$ & $\mathrm{x}$ & $\mathrm{X}$ & & $\mathrm{x}$ & $\mathrm{x}$ & $\mathrm{X}$ & $\mathrm{x}$ & 6 \\
\hline $\begin{array}{l}\text { Frank et al. } \\
\quad(2015)\end{array}$ & $\mathrm{X}$ & $\mathrm{X}$ & & $\mathrm{X}$ & $\mathrm{x}$ & $\mathrm{X}$ & & 5 \\
\hline $\begin{array}{l}\text { Gold et al. } \\
\text { (2010) }\end{array}$ & $\mathrm{x}$ & & & & $\mathrm{x}$ & $\mathrm{X}$ & & 3 \\
\hline $\begin{array}{l}\text { Harris et al. } \\
\quad(2016)\end{array}$ & $\mathrm{x}$ & $\mathrm{x}$ & $\mathrm{x}$ & $\mathrm{x}$ & $\mathrm{x}$ & $\mathrm{x}$ & $\mathrm{x}$ & 7 \\
\hline $\begin{array}{l}\text { Jennings et } \\
\text { al. (2011; } \\
\text { Study1) }\end{array}$ & $\mathrm{x}$ & & $\mathrm{x}$ & & $\mathrm{x}$ & $\mathrm{x}$ & & 4 \\
\hline $\begin{array}{l}\text { Jennings et } \\
\text { al. (2013) }\end{array}$ & $\mathrm{x}$ & $\mathrm{x}$ & $\mathrm{x}$ & $\mathrm{x}$ & $\mathrm{x}$ & $\mathrm{X}$ & & 6 \\
\hline
\end{tabular}


Reiser et

X

al. (2016)

Reiser \&

McCarthy

(2018)

Roeser et al. (2013)
X

X

X
X
X

X

X

X
X

$\mathrm{X}$
4

5

X

7 


\section{CHAPTER 2}

\section{METHOD}

\subsection{Participants}

Data for the current study were collected from teachers $(n=24)$ at a highperforming academic magnet high school in the Southeastern United States. Study participation was restricted to faculty members of this single school. The University of South Carolina IRB (Pro00071265) and school district IRB granted approval for intervention implementation and data collection procedures. Researchers presented on the study at the school's monthly faculty meeting one month prior to pre-intervention data collection to recruit participants for the study. Twenty-four faculty members expressed interest in participating in the bMBI and were randomly assigned to either the intervention group or the waitlist-control group. Of the 24 teachers that participated in both pre- and post-data collection, there were eighteen teachers, four guidance counselors, one school psychologist, and one assistant principal. The sample was predominantly female $(95.8 \%)$, White $(91.7 \%)$, and ranged from ages 25 to $70(M$ age $=$ $42.77 ; S D=11.25)$. Regarding education, $8 \%$ of participants reported having a bachelor's degree, $83 \%$ reported having a master's degree, and $8 \%$ reported having a doctoral degree. Teachers' years of experience ranged from 1 to 49 years $(M=15.58 ; S D=$ 11.98). Approximately 50\% of participants indicated that they had received some form of 
mental health service in the past (i.e., individual/group therapy, marriage counseling, etc.).

Given the novel brevity of our intervention design, we adopted stricter program completion criteria (i.e., attendance at three or more sessions; $75 \%$ ) than those used in past investigations (i.e., 33-50\% of program sessions). Only one participant in the intervention group did not meet this criterion and was subsequently excluded from analyses. Overall program attendance was exceptional (number of participants who attended all sessions $=7 / 11$ ) as all remaining participants included attended at least three sessions and, subsequently, were considered to have completed the program. See Table 2.1 for sample demographics.

\subsection{Procedure}

The study utilized a randomized waitlist-control design. Researchers consulted with the school's principal during the intervention development phase approximately six months prior to implementation in order to discuss interest, recruitment efforts, and possible barriers to implementation. Consultative feedback informed intervention design.

Participants were randomly assigned to either the intervention $(n=12)$ or waitlistcontrol $(n=12)$ group after completing pre-intervention data collection. Participants assigned to the intervention group participated in the program during the Winter/Spring (January - June) semester of 2018 and the waitlist-control group was offered the intervention during the Fall (August - November) semester of 2018. Researchers formally solicited participants' availability prior to scheduling all program sessions in an attempt to maximize intervention feasibility given teachers' extensive time demands. All 
sessions were held in the school's lecture hall and delivered during the afterschool hours. As compensation for participation, those in the intervention group who completed the program received six continuing education credits (CECs) and teachers in the waitlistcontrol condition received six CECs following their completion of the program in the following academic semester.

2.2.1 Intervention. The mindfulness program employed in this study was developed by two doctoral students and a developmental psychologist with consultative feedback from a clinical psychologist, a health psychologist, an educational psychologist, and the principal of the school. The program was sixteen weeks in duration and included one ninety-minute (90) session per month (i.e., four total sessions; six total contact hours). Program curriculum adhered to a cognitive-behavioral model of mindfulness comprised of three separate tenets: attentive awareness, receptive attitude, and intentionality (Renshaw \& O’Malley, 2014). Intentionality was operationalized and subsequently presented to participants as both a purposeful cultivation of mindfulness (Renshaw \& O'Malley, 2014; Brown et al., 2007) and setting intentions for this cultivation (Shapiro et al., 2006) in an effort to fully integrate all potentially beneficial aspects of this component into the intervention. Sessions one through three corresponded to these three tenants (i.e., session one: attentive awareness, etc.); an additional "integration" session constituted the fourth and final program session and focused explicitly on how these three constructs are conceptually and operationally interrelated. All participants were issued personalized workbooks facilitating and corresponding to various components of the program (i.e., didactics, journal entry, discussion, etc.). 
Each session followed a similar format: (1) a review of content from the previous meeting (for sessions 2 - 4); (2) a present-moment awareness exercise; (3) a didactic presentation pertaining to the individual session topic and its relation to self-regulation of stress management; (4) a mindfulness activity where participants model, operationalize, and practice the topic skill (e.g., receptive attitude); (5) a group discussion facilitated by open-ended questions; (6) a journal entry; and (7) a closing exercise (e.g., progressive muscle relaxation). Participants were encouraged to complete "in-between notes" (i.e., open-ended response in teacher workbooks to be completed between sessions) in an effort to promote practice and application of mindfulness skills outside of and between individual sessions. Additionally, the workbook contained an appendix with an additional set of exercises parceled by each session and corresponding with the particular skills practiced during the session. All of the curriculum was specifically adapted for teachers and program facilitators encouraged participants' exploration of how program content could inform both their personal and professional lives. Specific information on content and key themes of individual sessions is contained in Table 2.2.

\subsection{Measures}

Basic demographic information (i.e., age, race, gender, level of education, years of experience, etc.) and participants' past or current involvement with mental health services (i.e., individual/group therapy, marriage counseling, etc.) was obtained for all participants during data collection at pre-intervention.

2.3.1 Teacher Stress. Teachers' perceived stress levels were assessed using a self-report measure (Teacher Stress Inventory; TSI) consisting of 49 items rated on a 5- 
point Likert scale. The TSI contains the following 10 subscales: Time Management $(\alpha=$ .71); Work-Related Stressors $(\alpha=.64)$; Professional Distress $(\alpha=. .68)$; Discipline and Motivation $(\alpha=. .92)$; Professional Investment $(\alpha=.59)$; Emotional Manifestations $(\alpha=$ ..90); Fatigue Manifestations ( $\alpha=. .83)$; Cardiovascular Manifestations $(\alpha=.77)$;

Gastronomical Manifestations $(\alpha=. .76)$; and Behavioral Manifestations $(\alpha=.68)$.

Researchers adapted the original response choices for content clarification based on recommendations from pilot data (e.g., "Not noticeable/No strength" adapted to "Not true"). Although the measure demonstrated adequate psychometric properties in previous studies (Fimian \& Fastenau, 1990), the internal consistency of the Professional Investment Subscale in its original state was unacceptable in the current study (i.e., $\alpha \leq$ .60; DeVellis, 2016); however, after removing one item from the subscale (i.e., "I am not emotionally/intellectually stimulated on the job"), internal consistency improved to an acceptable range $(\alpha=.68)$. The internal consistency of the full scale in the current sample was excellent $(\alpha=.92)$.

Items on each subscale are summed and averaged to create a total subscale score; the ten subscale scores are also summed and averaged to create a total stress score. The current study analyzed the total stress score and each individual subscale separately to assess the separate dimensions of teacher stress, with higher scores indicative of greater amounts of perceived stress.

2.3.2 Teacher Burnout. Teachers' symptoms of burnout were assessed using the Maslach Burnout Inventory-Educators Survey (MBI-ES; Maslach, Jackson, \& Leiter, 1996). The measure consists of 22 items yielding the following three subscales: Emotional Exhaustion (nine items; $\alpha=.80$ ), Depersonalization (five items; $\alpha=.65$ ), and 
Reduced Personal Accomplishment (eight items; $\alpha=.83$ ). Internal consistency for the full scale was good $(\alpha=.87)$. Teachers rate their experiences relative to item content on a 7-point "fully-anchored" scale $(1=$ Never, $7=$ Every day $)$. The MBI has been used in the large majority of studies investigating symptoms of occupational burnout across numerous professions (Hastings, Horne, \& Mitchell, 2004). Past studies using the MBIES to measure burnout in teachers have demonstrated strong psychometric properties as the subscales yield strong correlations with theorized associated variables and internal consistency was adequate across all three subscales (Byrne, 2011; Kokkinos, 2006).

\subsubsection{Teacher Psychological Distress. The Symptom Assessment-45}

Questionnaire (SA-45; Davison et al., 1997) originally adapted as a short form of the Symptom Checklist-90 R (SCL-90-R; Derogatis, 1994), was used to assess teachers' psychological distress. The SA-45 is a brief assessment of psychologist distress evaluating symptoms contributing to different categories of psychological distress and has since been adapted for use with nonpatient populations (i.e., community samples; Maruish, Bershadsky, \& Goldstein, 1998). The scale utilizes a 5-point Likert scale (ranging from "Not at all" to "Extremely") on which respondents indicate the degree to which several psychiatric symptoms included in the SA-45 have bothered them over the past seven days.

The SA-45 yields the following nine subscales: Anxiety, Depression, ObsessiveCompulsion, Somatization, Phobic Anxiety, Hostility, Interpersonal Sensitivity, Psychoticism, and Paranoid Ideation. Responses to individual items also provide a summary score for the Global Severity Index (GSI). In addition to the GSI, the current study also analyzed the Anxiety $(\alpha=.68)$ and Depression $(\alpha=.88)$ subscales of the 
measure given they are the most commonly occurring symptoms in community samples (Kessler et al., 2003; English \& Campbell, 2019; Auerbach et al., 2018). Past studies using the SA-45 in both inpatient and community samples have demonstrated adequate psychometric properties (SAI, 1998) and internal consistency for the full scale in the current sample was excellent $(\alpha=.92)$. The instrument has been shown to effectively classify and discriminate between different diagnostic groups and functions similarly to other validated measures assessing similar constructs (Davison et al., 1997).

2.3.4 Teacher Mindfulness. Teacher mindfulness was assessed using a validated tool, the Five-Facet Mindfulness Questionnaire (FFMQ), that is designed to measure aspects of mindfulness that an individual can possess or learn through mindfulness training. The FFMQ consists of 39 items utilizing a 5-point Likert Scale. The scale measures five skills, each its own respective subscale, that previous research indicates are indicative of effective mindfulness practice: Observing $(\alpha=.86)$, Describing $(\alpha=.91)$, Acting with Awareness $(\alpha=.79)$, Non-reactivity $(\alpha=.93)$, and Nonjudgement of Inner Experience ( $\alpha=.81$ ) (Baer et al., 2008). In past research, internal consistency ratings in samples of both regularly meditating and non-meditating individuals ranged from sufficient to excellent, and all five facets were significantly correlated with mediation experience, psychological adjustment, and well-being (Baer et al., 2008). Internal consistency for the full scale in the current sample was also excellent $(\alpha=.91)$.

2.3.5 Cortisol Response. Past research has established salivary cortisol as an accurate and commonly utilized reflection of the actual amount of cortisol secreted within the body, making it a valid physiological marker of the human stress response (Scassellati, Bonvicini, Faraone, \& Gennarelli, 2012). Additionally, the CAR has been 
validated as a reliable and minimally-invasive endocrine marker for the human stress response that allows for more effective control of collection, which mitigates the effects caused by variable levels throughout the day (Federenko, Nagamine, Hellhammer, Wadhwa, \& Wüst, 2004; Pruessner et al., 1997).

Starstedt Salivettes ${ }^{\circledR}$ were distributed to all participants. Each participant was asked to provide two salivary cortisol samples upon awakening on two consecutive days at baseline and post-intervention (i.e. eight total samples per participant). Researchers instructed participants on how to provide their saliva sample immediately upon waking and 30 minutes thereafter on these days, as previous literature suggests this is appropriate practice (Hellhammer et al., 2007). Participants were also instructed to record their time of awakening and sample collection time. This is recommended practice as it ensures differences in salivary cortisol concentrations are not attributable to the diurnal pattern of fluctuation, which research shows is particularly volatile during the first hour after awakening (Hanrahan, McCarthy, Kleiber, Lutgendorf, \& Tsalikian, 2006; Hellhammer et al., 2007) and sensitive to anticipatory next day stress (Fries et al., 2009).

Participants were instructed to place the swab in their mouth and to chew for 2-3 minutes to ensure an adequate amount of saliva is collected. After the sample was collected, participants then placed the swab into a container pre-labeled with their relevant identifying information and time collection point (e.g., baseline); and then stored their sample in their freezer. To ensure the samples do not mold, participants were asked to return their sample to research staff four days after Salivette distribution, at which point the sample was stored in a freezer at $-20^{\circ} \mathrm{C}$ to preserve it until further analysis. 
Cortisol samples were shipped to the Biochemical Laboratory of the Department of Biological and Clinical Psychology at Universität Trier in Germany for analysis. After thawing, saliva samples centrifuged at $2000 \mathrm{~g}$ for 10 minutes, which resulted in a clear supernatant of low viscosity. Duplicate analysis utilized 100ul of saliva. Cortisol levels were determined employing a competitive solid phase time-resolved fluorescence immunoassay with flouromeric end point detection (DELFIA). Ninety-six-well-Maxisorb microtiterplates were coated with polyclonal swine anti-rabbit immunoglobulin. After an incubation period of $48 \mathrm{~h}$ at $4{ }^{\circ} \mathrm{C}$ plates were washed three times with wash buffer $(\mathrm{pH}$ $7,4)$.

In the next step, the plates were coated with a rabbit anti-cortisol antibody and incubated $48 \mathrm{~h}$ at $4{ }^{\circ} \mathrm{C}$. Synthetic saliva mixed with cortisol in a range from 0 to 100 $\mathrm{nmol} / \mathrm{L}$ served at standards. Standards, controls (saliva pools), and samples were given in duplicate wells. Fifty nmol/L of biotin conjugated cortisol was added and after 30min of incubation the non-binding cortisol/bioton-conjugated cortisol was removed by washing (3x) 200 ul europium-streptavidin (Perkin Elmerc, Liefe science Turku, Finland) was added to each well and after 30 minutes and 6 times of washing, 200 ul enhancement solution was added (Pharmacia, Freiburg, Germany). Within 15 minutes on a shaker, the enhancement solution induced the fluorescence which can be detected DELFIAFluorometer (Wallac, Turku, Finland). Using a computer-controlled program, a standard curve was generated, and the cortisol concentration of the samples were calculated. The intra-assay coefficients of variation were between $4.0 \%$ and $6.7 \%$ and the corresponding inter-assay coefficients of variation were between 7.1\%-9.0\% (Dressendorfer, Kirschbaum, Rhode, Stahl, \& Strasburger, 1992). 


\subsection{Analytic Procedures}

2.4.1 Sample equivalence. All analyses were conducted using SPSS v. 21.0 (IMB Corp, 2017). First, following randomization, chi-square analyses were used to compare the intervention and waitlist-control groups with respect to gender, age, race, years of experience, level of education, and history of receiving mental health services.

\subsubsection{Pre-intervention equivalence on teacher outcomes and mechanism of}

change measures. Independent samples $t$-tests were used to examine the equivalence of intervention and waitlist-control groups on measures of primary teacher outcomes and mechanism of change at pre-intervention.

\subsubsection{Relations between primary teacher outcomes and mechanism of change}

variables. Bivariate correlations for primary teacher outcomes and mechanism of change variables were calculated to examine the relations between these variables following the implementation of the intervention.

\subsubsection{Effect of bMBI on teacher outcome measures. Participants' cortisol} responses were categorized as adaptive (i.e., within 38-75\% of an increase in response from waking to 30 minutes post-waking; Pruessner et al., 1997; Fries et al., 2009) or maladaptive (i.e., exhibiting a blunted response that falls below this range or a heightened response that is above this range). Chi-square analyses were used to determine whether there were significant changes from pre- to post-intervention regarding the number of participants that were categorized as having either an adaptive or maladaptive response in the intervention and waitlist-control groups, respectively. Cramer's $V$ was calculated to determine the magnitude of the change from pre- to post-intervention in the intervention and waitlist-control groups, respectively. The larger the Cramer's $V$ value, the more 
substantial the difference is in expected values of cells representing categories of CAR from pre- to post-intervention. Given the vast heterogeneity in the CAR across individuals and variability in responses to stress and burnout (Wust et al., 2000; Miller et al., 2007; Pruessner et al., 1999), statistical analyses in samples with low power often fail to identify changes to this marker of stress. Therefore, these data were also analyzed further at a qualitative level to examine individual changes to CAR from pre- to postintervention

Paired samples $t$-tests were used to examine changes from pre- to postintervention in the intervention and waitlist-control groups, respectively, on all teacher outcome variables including the following: TSI (including the full composite scale and the 10 separate subscales), MBI-ES (including the full scale and the three separate subscales), and the SA-45 (including the full scale [i.e., the General Symptom Index] and the Anxiety and Depression subscales). Cohen's $d$ effect sizes were calculated for the change from pre- to post-intervention in the intervention and waitlist-control groups, respectively. The direction of Cohen's $d$ was corrected such that larger positive $d$ s indicate greater improvements to markers of stress and well-being. Given the large number of comparisons included in this preliminary aim, the current study also utilized the Holm-Bonferroni Method to demonstrate the nature of the findings when correcting for family-wise error.

2.4.5 Dimensions of Mindfulness as Mechanisms of Change. Paired samples $t$ tests were used to examine changes from pre- to post-intervention in the intervention and waitlist-control groups, respectively, on the full mindfulness scale (FFMQ) and the five separate subscales (i.e., Observe, Describe, Act with Awareness, Nonjudgment, and Non- 
reactivity) of the measure. Cohen's $d$ effect sizes were calculated for the change from pre- to post-intervention in the intervention and waitlist-control groups, respectively, and the direction of Cohen's $d$ was corrected such that larger positive $d$ s indicate improvements in mindfulness.

Change scores from pre- to post-intervention were calculated for all teacher outcome variables in the intervention group, including CAR, TSI, MBI-ES, GSI, and for teacher mindfulness (i.e., the FFMQ, including the five separate dimensions of mindfulness). Bivariate correlations (i.e., one-tailed tests of significance) were used to examine the degree to which changes in each dimension of mindfulness from pre- to post-intervention is associated with improvements in teacher outcomes. 
Table 2.1. Sociodemographic characteristics of the current study by condition.

\begin{tabular}{|c|c|c|c|c|}
\hline $\begin{array}{l}\text { Sociodemographic } \\
\text { characteristic }\end{array}$ & $\begin{array}{l}\text { Intervention }(n \\
\quad=11) \\
n(\%)\end{array}$ & $\begin{array}{l}\text { Waitlist-control ( } n \\
\qquad \begin{array}{c}=12) \\
n(\%)\end{array}\end{array}$ & $\chi^{2}(\mathrm{df})$ & $p$ \\
\hline \multicolumn{5}{|l|}{ Gender } \\
\hline Female & $10(91)$ & $11(100)$ & $\begin{array}{c}\chi^{2}(1)= \\
1.140\end{array}$ & .286 \\
\hline Male & $1(9)$ & $0(0)$ & & \\
\hline \multicolumn{5}{|l|}{ Age } \\
\hline $20-29$ & $1(9)$ & $2(17)$ & $\begin{array}{c}\chi^{2}(4)= \\
2.161\end{array}$ & .706 \\
\hline $30-39$ & $3(27)$ & $4(33)$ & & \\
\hline $40-49$ & $2(18)$ & $3(25)$ & & \\
\hline $50-59$ & $4(36)$ & $2(17)$ & & \\
\hline $60-69$ & $0(0)$ & $0(0)$ & & \\
\hline $70-79$ & $0(0)$ & $1(8)$ & & \\
\hline \multicolumn{5}{|l|}{ Race } \\
\hline White & $10(91)$ & $11(92)$ & $\begin{array}{l}\chi^{2}(2)= \\
2.008\end{array}$ & .366 \\
\hline Black & $0(0)$ & $1(8)$ & & \\
\hline Other & $1(9)$ & $0(0)$ & & \\
\hline \multicolumn{5}{|l|}{ Years of experience } \\
\hline $0-9$ & $3(27)$ & $6(54)$ & $\begin{array}{c}\chi^{2}(3)= \\
6.254\end{array}$ & .100 \\
\hline $10-19$ & $4(36)$ & $2(18)$ & & \\
\hline $20-29$ & $4(36)$ & $1(9)$ & & \\
\hline $30+$ & $0(0)$ & $3(27)$ & & \\
\hline \multicolumn{5}{|l|}{ Level of education } \\
\hline Bachelor's Degree & $0(0)$ & $1(8)$ & $\begin{array}{l}\chi^{2}(2)= \\
4.017\end{array}$ & .134 \\
\hline Master's Degree & $9(82)$ & $11(92)$ & & \\
\hline Doctorate Degree & $2(18)$ & $0(0)$ & & \\
\hline \multicolumn{5}{|l|}{$\begin{array}{l}\text { Received past mental health } \\
\text { services }\end{array}$} \\
\hline Yes & $5(45)$ & $7(58)$ & $\begin{array}{c}\chi^{2}(1)= \\
.381\end{array}$ & .537 \\
\hline No & $6(55)$ & $5(42)$ & & \\
\hline
\end{tabular}


Table 2.2. Overview of content and key themes in bMBI curriculum.

\begin{tabular}{|c|c|c|c|}
\hline Session & $\begin{array}{l}\text { Mindfulness } \\
\text { tenant }\end{array}$ & Activities & Key session themes \\
\hline 1 & $\begin{array}{l}\text { Attentive } \\
\text { awareness }\end{array}$ & $\begin{array}{l}\text { Introduction to mindfulness, program } \\
\text { facilitators, teacher workbooks; group } \\
\text { rules; sensory experience exercise; } \\
\text { participant goals for program } \\
\text { participation; didactics regarding } \\
\text { attentive awareness (i.e., types of } \\
\text { attention, attentional demands of } \\
\text { teachers and students); topic exercise } \\
\text { and reflection; group discussion; } \\
\text { journal entry; progressive muscle } \\
\text { relaxation }\end{array}$ & $\begin{array}{l}\text { Identifying participants' goals } \\
\text { for program participation; daily } \\
\text { attentional demands; effects of } \\
\text { chronically not cultivating } \\
\text { attentive awareness (i.e., "on } \\
\text { auto-pilot") }\end{array}$ \\
\hline 2 & $\begin{array}{l}\text { Receptive } \\
\text { attitude }\end{array}$ & $\begin{array}{l}\text { Review of attentive awareness, session } \\
\text { one journal entry, in-between notes; } \\
\text { introductory exercise; didactics } \\
\text { regarding receptive attitude, (i.e., } \\
\text { approaching situations with openness, } \\
\text { curiosity, and acceptance); topic } \\
\text { exercise and reflection; group } \\
\text { discussion; journal entry; loving } \\
\text { kindness meditation }\end{array}$ & $\begin{array}{l}\text { Operationalizing "approach } \\
\text { with curiosity, openness, and } \\
\text { acceptance"; effects of non- } \\
\text { receptive attitude (i.e., } \\
\text { experiential avoidance); } \\
\text { willingness; self-compassion }\end{array}$ \\
\hline
\end{tabular}


Intentionality

$4 \quad$ Integration
Review of receptive attitude, session two journal entry, in-between notes; review of program goals; introductory exercise; didactics regarding intentionality (i.e., values-based discussion of effort, psychological grit); topic exercise and reflection; group discussion; journal entry; progressive muscle relaxation

Review of intentionality, session three journal entry, in-between notes; topic exercise and reflection; video presentation; discussion of how three tenants of mindfulness interrelate; discussion of continued application of mindfulness strategies (i.e. barriers, future goals) concluding exercise
Review of program and participant goals; identifying participant values; compassion as a value; competing thoughts and distressing emotions as barriers to intentional behavior; development of SMART goal

\section{Operationalizing and} integrating tenants of mindfulness in personal and professional life; identification of barriers to continued practice 


\section{CHAPTER 3}

\section{RESULTS}

\subsection{Sample Descriptives}

Analyses indicated no significant differences across experimental groups with respect to gender, age, race, years of experience, level of education, or history of receiving mental health services (see Table 2.1). There was a significant difference between the intervention group and waitlist-control group regarding the number of teachers with an adaptive (as opposed to maladaptive) CAR at baseline $\left(\chi^{2}(1, n=23)=\right.$ $4.537 p=.033$; see Table 3.1) such that there were more teachers with an adaptive response in the control group than the intervention group at baseline. No additional significant differences between experimental groups were observed on any teacher outcome measure or mechanism of change measure at pre-intervention. Descriptive statistics indicated that teachers in both the intervention group (mean $=3.37, \mathrm{SD}=.43$, range $=1.49)$ and waitlist-control group $($ mean $=3.33, \mathrm{SD}=.46$, range $=1.62)$ reported having generally average mindfulness skills at baseline suggesting they would benefit from engaging in more practice.

Bivariate correlations were conducted on all study variables for the full sample at post-intervention to examine initial relations between mindfulness domains and teacher outcomes. These analyses indicated significant relations between the full mindfulness 
scale (FFMQ) and the full scales for teacher stress (TSI; $r(22)=-.530, p=.009)$ and burnout (MBI-E; $r(22)=-.428, p=.042$; see Table 3.2) but not for physiological measures of stress (CAR) or psychological distress (GSI of the SA-45). Additionally, some mindfulness subscales were found to be associated with positive teacher outcomes at post-intervention, with the Nonjudgment subscale of the FFMQ significant and inversely related to teacher psychological distress (GSI; $(r(22)=-.552, p=.006)$, and the Non-reactivity subscale of the FFMQ was significantly and inversely related to teacher's self-report of stress (TSI; $(r(22)=-.602, p=.002$; see Table 3.2). These findings demonstrate that mindfulness at post-intervention, and some of the separate mindfulness domains, are related to primary teacher outcomes at post-intervention. See Table 3.2 for details regarding relations amongst domains of mindfulness and teacher outcome variables, respectively. Additionally, bivariate correlations amongst change score variables from pre- to post-intervention (computed to address the research question associated with Aim 2) indicated that the changes in the CAR were significantly related to changes in the TSI $(r(10)=-566, p=.035$; see Table 3.3), which suggests that teacher's self-reported stress (TSI) was a convergent proxy for this physiological marker of stress.

\subsection{Aim 1: Efficacy of bMBI on Teacher Stress and Burnout}

\subsubsection{Effect of bMBI on teacher stress.}

3.2.1.1 Physiological Stress. Chi-square analyses indicated no significant changes from pre- to post-intervention for the intervention group $\left(\chi^{2}(1, n=11)=.413, p=.521\right.$; see Table 3.1), but there was a small effect ( $V=.193$; Cohen, 2013) indicating a need for 
further examination to determine the nature of these changes. Likewise, there was also no significant effect for CAR from pre- to post-intervention in the waitlist-control group $\left(\chi^{2}\right.$ $(1, n=12)=1.500, p=.221)$, but there was a medium effect $(V=.354)$. As outlined, these analyses were further examined qualitatively since the heterogeneity in cortisol responses often leads to difficulties in identifying statistically meaningful effects in smaller samples (Wust et al., 2000; Miller et al., 2007; Pruessner et al., 1999), and this examination allows us to further determine the directional nature of shifts in cortisol responses from pre- to post-intervention in each group.

Figure 3.1 represents the CAR of each individual at pre- and post-intervention, and Figure 3.2 represents the average cortisol values at both waking and 30-minutes postwaking over the course of both days at pre- and post-intervention (see Table 3.4 for mean values in each group). At pre-intervention, only one of 11 teachers in the intervention group exhibited a CAR within the adaptive range (i.e., 38-75\% increase from waking levels). The other 10 teachers demonstrated a maladaptive response (six teachers with blunted response; four teachers with heightened response). There was a positive shift at post-intervention in the intervention group as three teachers exhibited an adaptive response (two moved from blunted to adaptive and one moved from heighted to adaptive). Eight teachers continued to demonstrate a maladaptive response (seven teachers exhibited blunted responses; one teacher exhibited a heightened response). In summary, examining this shift from pre- to post-intervention more closely indicates that three teachers moved from exhibiting a maladaptive CAR to an adaptive response by post-intervention while only one teacher moved from having an adaptive response to having a maladaptive (i.e., blunted for this individual) response (see Table 3.5). These 
physiological profiles are consistent with self-reported levels of teacher stress and burnout (see Table 3.6) in the intervention group whereby those experiencing high amounts of stress and burnout at baseline showed improvements following the intervention.

By comparison, three teachers in the waitlist-control group had shifted from demonstrating an adaptive response at baseline to a maladaptive response at postintervention, and only one teacher had moved from exhibiting a maladaptive response to an adaptive response (see Table 3.5). Six of 12 teachers presented with an adaptive response at pre-intervention. Of the six teachers who presented with a maladaptive response at baseline, four teachers demonstrated a blunted response and two demonstrated a heightened response. At post-intervention, only four teachers exhibited an adaptive CAR at post-intervention. Of the remaining eight teachers who presented with a maladaptive response at post-intervention, six demonstrated a blunted response, and two teachers exhibited a heightened response. Thus, a closer qualitative examination of these values, in conjunction with the medium effect observed in the control group $(\mathrm{V}=.354)$, suggests there is a maladaptive trend in physiological functioning for those in the control group.

3.2.1.2 Self-Reported Stress. Results from paired samples $t$-tests indicated significant reductions on self-reported teacher stress (i.e., TSI full scale) for the intervention group from pre- to post-intervention $(t(10)=5.027, p=.001$; see Table 3.6). No significant reductions in self-reported stress from pre- to post-intervention were observed for the waitlist-control group $(t(11)=.803, p=.439)$. There was a large effect size observed for reduction in perceived stress in the intervention group $(d=1.54)$. 
Among the 10 subscales of the TSI, significant changes from pre- to postintervention were observed for the following five subscales: Time Management $(t(10)=$ $3.474, p=.006)$; Work-Related Stressors $(t(10)=3.382, p=.007)$; Professional Distress $(t(10)=3.064, p=.012) ;$ Professional Investment $(t(10)=2.451, p=.034)$; and Fatigue Manifestations $(t(10)=2.335, p=.042)$. Of these, large effect sizes were observed for Time Management $(d=1.06)$, Work-Related Stressors $(d=1.03)$, and Professional Distress $(d=.92)$, and medium effect sizes were observed for Professional Investment ( $d$ $=.75)$ and Fatigue Manifestations $(d=.71)$. Of note, there was also a medium effect size observed for Emotional Manifestations $(d=.66)$, but the paired-samples $t$-tests did not reach significance $(t(10)=2.162, p=.056)$. All remaining subscales did not demonstrate significant change (see Table 3.6). There were no significant changes observed on any of the subscales for the waitlist-control group.

\subsubsection{Effect of bMBI on teacher burnout. The intervention group reported} significant reductions in symptoms of burnout (i.e., the full scale of the MBI-ES) from pre- to post-intervention $(t(10)=3.012, p=.013$; see Table 3.6), but no significant reductions in burnout from pre-to post-intervention were observed for the waitlist-control group $(t(11)=.771, p=.457)$. A large effect was observed for symptoms of burnout $(d=$ $.92)$ in the intervention group from pre- to post-intervention.

Among the three subscales of the MBI-ES, the intervention group reported significant reductions from pre- to post-intervention on only the Emotional Exhaustion subscale of the MBI-ES $(t(10)=4.001, p=.003)$ for which there was a large effect size $(d=1.21)$. There was a small effect for the Depersonalization subscale $(d=.24)$, but this did not reach significance $(t(10)=0.820, p=.432)$. There was also no significant effect 
for the Personal Accomplishment subscale $(t(10)=.540, p=.601)$. As expected, there were no significant effects observed on the MBI-ES subscales in the control group from pre- to post-intervention).

3.2.3 Effect of bMBI on teacher psychological distress. Paired samples $t$-tests demonstrated a significant improvement on the Depression subscale of the SA-45 for the intervention group from pre- to post-intervention $(t(10)=2.352, p=.040)$ with a medium effect size $(d=.71)$, but there was not a significant improvement for the waitlist-control group $(t(11)=1.764, p=.105)$. However, the bMBI had less of an impact on other indicators of psychological distress. There were no significant improvements in general psychological distress (i.e., the full GSI scale of the SA-45) from pre- to post-intervention observed for the intervention $(t(10)=1.139, p=.281)$ or waitlist-control group $(t(11)=$ $1.488, p=.165)$. The effect size indicates there were small improvements for the intervention group $(d=.34)$ from pre- to post-intervention. There was also not a significant improvement in anxiety from pre- to post-intervention for either the intervention group $(t(10)=1.009, p=.337)$ or the waitlist-control group $(t(11)=1.024, p$ $=.328$ ). Only a small effect size was observed in both groups (i.e., $d=.30$ in both groups).

3.2.4 Correcting for family-wise error. The Holm-Bonferroni Method was used to account for the increased risk of type I error when conducting multiple tests on outcome variables. This method is recommended for studies with low power trying to control for multiple comparisons (Abdi, 2010; Aickin \& Gensler, 1996). The HolmBonferroni Method involves sorting p-values of statistically significant tests (i.e., $P_{(1)}$, $\left.P_{(2)}, \ldots P_{(m)}\right)$ in a given family of hypotheses (i.e., $\left.H_{(l)} \ldots H_{(m)}\right)$. These hypotheses are then 
re-tested sequentially using the formula $P_{(h)} \leq \frac{\alpha}{m-h+1}$ until one of the tests fails to reject the $H_{0}$ with its corrected $\alpha$.

Accordingly, the paired samples $t$-tests for outcomes variables from pre- to postintervention were ordered as follows: TSI full scale $(p=.001)$, MBI-ES Emotional Exhaustion subscale $(p=.003)$; TSI Time Management subscale $(p=.006)$; TSI WorkRelated Stressors subscale $(p=.007)$; TSI Professional Distress subscale $(p=.012)$; MBI-ES full scale ( $p=.013)$; TSI Professional Investment subscale $(p=.034)$; SA-45 Depression subscale $(p=.040)$; and the TSI Fatigue Manifestations subscale $(p=.042)$. Based on the corrected tests, the TSI full scale $(.001<.006)$, MBI-ES Emotional Exhaustion subscale $(.003<.006)$, TSI Time Management subscale $(.006<.007)$, and TSI Work-Related Stressors subscale $(.007<.008)$ remained significant. However, the Professional Distress, Professional Investment, and Fatigue Manifestations subscales of the TSI were no longer significant, and the reductions in burnout and depression no longer reached significance.

\subsection{Aim 2: Dimensions of Mindfulness as Mechanisms of Change}

3.3.1 Effect of bMBI on teacher mindfulness. There was not a significant improvement in teacher mindfulness from pre- to post-intervention in the intervention group $(t(10)=-1.798, p=.102)$ or waitlist-control group $(t(11)=1.139, p=.281$; see Table 3.7). However, a medium effect on mindfulness was observed for the intervention $\operatorname{group}(d=.56)$.

Among the five dimensions of mindfulness, only the Describe subscale showed a significant improvement from pre- to post-intervention for the intervention group $(t(10)=$ 
$-2.533, p=.030)$. There was a medium effect size on this dimension $(d=.76)$. Despite not reaching significance, there was also a medium effect size for the Act with Awareness dimension of mindfulness $(d=.51)$ and a small effect size for the Observe dimension of mindfulness $(d=.33)$ for the intervention group. There were no significant effects on any dimension of mindfulness in the waitlist-control group.

\subsubsection{Relations between changes in dimensions of mindfulness on changes in}

teacher outcomes. Bivariate correlations amongst changes scores in mindfulness (including its five separate dimensions) and outcome variables did not indicate any significant relations (one-tailed tests; see Table 3.3). However, the full mindfulness scale (FFMQ) showed medium-sized inverse relations with the full teacher stress scale (TSI; $r(10)=-.381, p=.124)$ full burnout scale (MBI-ES; FFMQ $(r(10)=-.353, p=.144)$, and the full general symptom scale (GSI of the SA-45; $r(10)=-.482, p=.066$ ).

Additionally, several of the specific dimensions of mindfulness demonstrated moderate-to-large correlation coefficients to teacher outcomes (see Table 3.3). For example, the changes from pre- to post-intervention for the Describe subscale of the FFMQ showed a large inverse relation with changes on the full teacher stress scale (TSI); $r(10)=-.505, p=.057$; see Table 3.3). Similarly, the changes in the Act with Awareness subscale of the FFMQ showed a medium-sized inverse relation with and the TSI $(r(10)=$ -.428, $p=.069)$. There were medium-sized inverse relations between the Describe subscale and the full burnout scale (i.e., the MBI-ES; $r[10]=-.401, p=.111$ ) and the Act with Awareness subscale and burnout $(r(10)=-. .478, p=.069)$. Four of the five subscales of the FFMQ also demonstrated a medium effect on the full psychological distress scale (i.e., the GSI of the SA-45): Observe $(r(10)=-.467, p=.074)$; Act with 
Awareness $(r(10)=-.317, p=.171)$; Nonjudgment $(r(10)=-.308, p=.179)$; and Nonreactivity $(r(10)=-.477, p=.069)$. 
Table 3.1 Chi square analyses for cortisol awakening response.

\begin{tabular}{|c|c|c|c|c|c|c|c|}
\hline $\begin{array}{l}\text { Sociodemographic } \\
\text { characteristic }\end{array}$ & $\begin{array}{c}\text { Adaptive } \\
\text { CAR at Pre- } \\
\text { Intervention }\end{array}$ & $\begin{array}{l}\text { Maladaptive } \\
\text { CAR at Pre- } \\
\text { Intervention }\end{array}$ & $\begin{array}{l}\text { Adaptive } \\
\text { CAR at Pre- } \\
\text { Intervention }\end{array}$ & $\begin{array}{l}\text { Maladaptive } \\
\text { CAR at Post- } \\
\text { Intervention }\end{array}$ & $\begin{array}{l}\chi^{2} \\
(\mathrm{df})\end{array}$ & $p$ & Cramer's $V$ \\
\hline $\begin{array}{l}\text { Intervention Group ( } n \\
=11 \text { ) (Adj. } \\
\text { Standardized } \\
\text { Residuals) }\end{array}$ & $1(-.6)$ & $10(.6)$ & $3(-.6)$ & $8(.6)$ & $\begin{array}{c}\chi^{2}(1)= \\
.413\end{array}$ & .521 & .194 \\
\hline $\begin{array}{l}\text { Waitlist-Control } \\
\text { Group }(n=12) \text { (Adj. } \\
\text { Standardized } \\
\text { Residuals) }\end{array}$ & $6(1.2)$ & $6(-1.2)$ & $4(1.2)$ & $8(-1.2)$ & $\begin{array}{c}\chi^{2}(1)= \\
1.500\end{array}$ & .273 & .354 \\
\hline
\end{tabular}


Table 3.2 Correlations between mindfulness and primary outcome variables at post-intervention.

\begin{tabular}{|c|c|c|c|c|c|c|c|c|c|}
\hline Variable & 1 & 2 & 3 & 4 & 5 & 6 & 7 & 8 & 9 \\
\hline 1 - FFMQ Full & - & & & & & & & & \\
\hline 2 - FFMQ Observe & $.740^{*}$ & - & & & & & & & \\
\hline 3 - FFMQ Describe & $.532 *$ & $.469 *$ & - & & & & & & \\
\hline $\begin{array}{c}\text { 4-FFMQ Act with } \\
\text { Awareness }\end{array}$ & $.711 *$ & .339 & .406 & - & & & & & \\
\hline 5 - FFMQ Nonjudgment & $.550 *$ & .137 & -.246 & .241 & - & & & & \\
\hline 6 - FFMQ Non-reactivity & $.863^{*}$ & $.600 *$ & .435 & $.555^{*}$ & $.413^{*}$ & - & & & \\
\hline $7-\mathrm{CAR}$ & -.082 & -.176 & -.164 & .096 & .003 & -.026 & - & & \\
\hline $8-\mathrm{TSI}$ & $-.530 *$ & -.277 & -.163 & -.349 & -.396 & $-.602 *$ & .030 & - & \\
\hline $9-\mathrm{MBI}$ & $-.428 *$ & -.204 & -.273 & -.265 & -.374 & -.272 & .077 & $.693^{*}$ & - \\
\hline
\end{tabular}


$*$ Correlations at post-intervention significant at $p<.05$. $n=12$ 
Table 3.3 Dimensions of mindfulness as mechanisms of change.

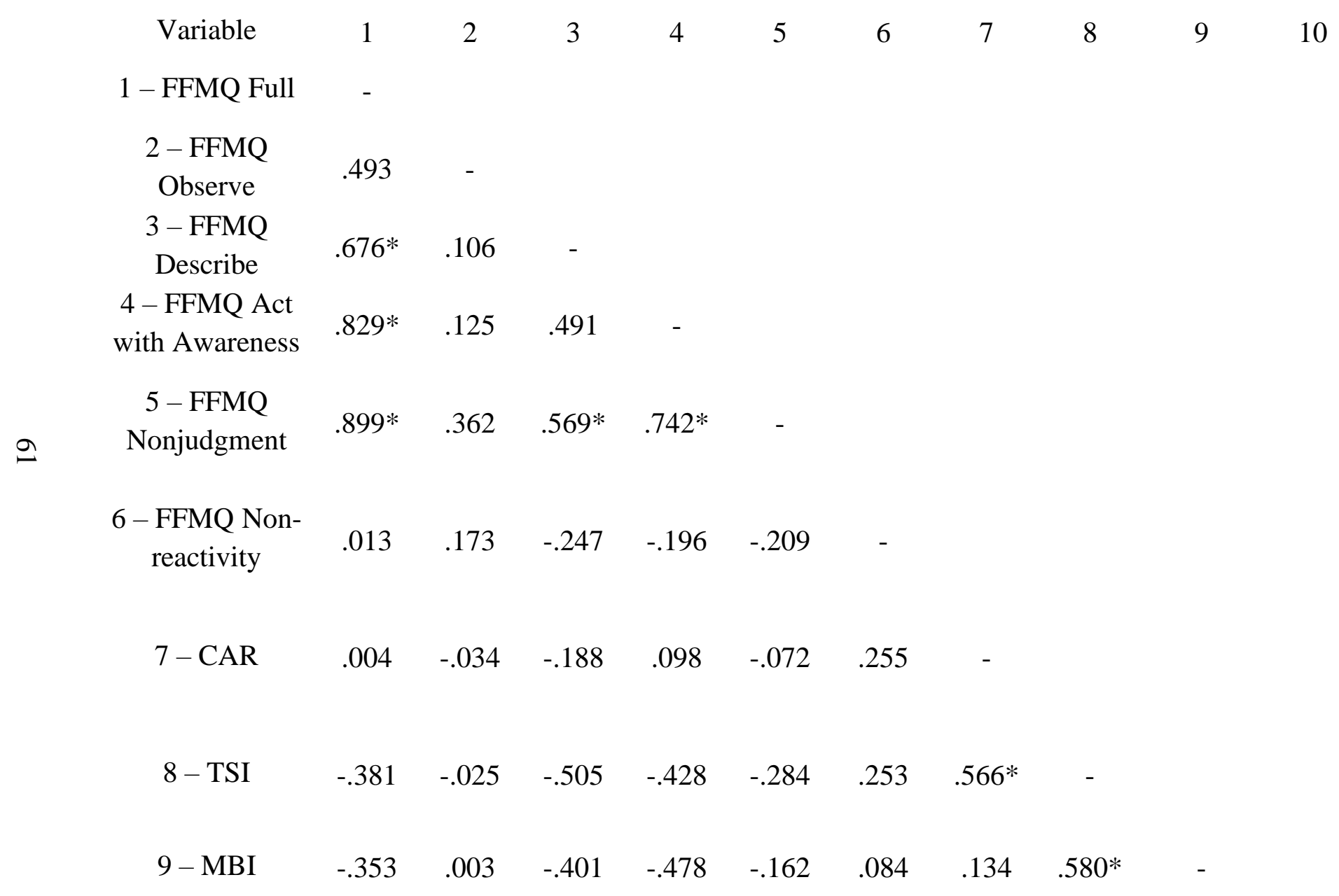


$10-\mathrm{GSI}$

$-.482$

$-.467$

$-.087$

$-.317$

$-.308$

$-.477$

.406

.343

.336

* Correlations for change scores in Intervention group $(n=11)$ significant at $p<.05$ (one-tailed). 
Table 3.4 Mean cortisol response values.

\begin{tabular}{|c|c|c|c|c|c|c|c|c|}
\hline \multirow[b]{3}{*}{ Outcome measure (scale) } & \multicolumn{4}{|c|}{ Intervention $(n=11)$} & \multicolumn{4}{|c|}{ Waitlist-control $(n=12)$} \\
\hline & \multicolumn{2}{|c|}{ Pre } & \multicolumn{2}{|c|}{ Post } & \multirow[b]{2}{*}{$M$} & \multicolumn{2}{|c|}{ Pre } & \multirow{2}{*}{$\frac{\text { Post }}{\mathrm{SD}}$} \\
\hline & $M$ & SD & $M$ & SD & & $\mathrm{SD}$ & $M$ & \\
\hline Cortisol Awakening & 4.18 & 5.84 & 1.63 & 3.87 & 4.44 & 4.41 & 3.97 & 6.37 \\
\hline Response (CAR; nmol/L) & & & & & & & & \\
\hline $\begin{array}{c}\text { Cortisol T1 (immediately } \\
\text { upon waking) }\end{array}$ & 9.00 & 3.77 & 10.62 & 3.19 & 9.60 & 4.03 & 11.01 & 4.98 \\
\hline $\begin{array}{c}\text { Cortisol T2 (30min post- } \\
\text { waking) }\end{array}$ & 13.18 & 5.44 & 12.25 & 3.69 & 14.04 & 3.71 & 14.98 & 7.50 \\
\hline
\end{tabular}


Table 3.5 Shifts in teachers' CAR from pre- to post-intervention.

\begin{tabular}{ccccc}
\hline $\begin{array}{c}\text { Sociodemographic } \\
\text { characteristic }\end{array}$ & $\begin{array}{c}\text { Participants } \\
\text { Remaining } \\
\text { Adaptive }\end{array}$ & $\begin{array}{c}\text { Participants } \\
\text { Moving from } \\
\text { Adaptive to } \\
\text { Maladaptive }\end{array}$ & $\begin{array}{c}\text { Participants } \\
\text { Remaining } \\
\text { Maladaptive }\end{array}$ & $\begin{array}{c}\text { Participants Moving } \\
\text { from Maladaptive to } \\
\text { Adaptive }\end{array}$ \\
\hline $\begin{array}{c}\text { Intervention Group }(n=11) \\
\text { Waitlist-Control Group }(n=\end{array}$ & 0 & 1 & 7 & 3 \\
$12)$ & 3 & 3 & 5 & 1
\end{tabular}


Table 3.6 Mean comparisons of teacher outcome variables by group.

\begin{tabular}{|c|c|c|c|c|c|c|c|c|c|c|}
\hline \multirow[b]{3}{*}{ Outcome measure (scale) } & \multicolumn{5}{|c|}{ Intervention $(n=11)$} & \multicolumn{5}{|c|}{ Waitlist-control $(n=12)$} \\
\hline & \multicolumn{2}{|c|}{ Pre } & \multicolumn{2}{|c|}{ Post } & \multirow[b]{2}{*}{$d$} & \multicolumn{2}{|c|}{ Pre } & \multicolumn{2}{|c|}{ Post } & \multirow[b]{2}{*}{$d$} \\
\hline & $M$ & SD & $M$ & SD & & $M$ & SD & $M$ & SD & \\
\hline TSI total (1-5) & 2.65 & .49 & $2.34 *$ & .50 & 1.54 & 2.94 & .50 & 2.85 & .48 & .22 \\
\hline Time Management & 3.82 & .63 & $3.36^{*}$ & .62 & 1.06 & 3.76 & .44 & 3.61 & .61 & .22 \\
\hline Work-Related Stressors & 3.20 & .64 & $2.63 *$ & .72 & 1.03 & 3.57 & .47 & 3.36 & .75 & .32 \\
\hline Professional Distress & 2.67 & .92 & $2.00 *$ & .69 & .92 & 3.00 & .83 & 3.15 & 1.03 & .22 \\
\hline Discipline and Motivation & 2.12 & .90 & 2.27 & .77 & .28 & 3.35 & .75 & 3.40 & 1.01 & -.07 \\
\hline Professional Investment & 2.32 & .86 & $1.95^{*}$ & .57 & .75 & 2.38 & .83 & 2.27 & .57 & .02 \\
\hline Emotional Manifestations & 3.00 & 1.03 & 2.65 & 1.02 & .66 & 2.90 & 1.15 & 2.81 & 1.45 & .14 \\
\hline Fatigue Manifestations & 2.51 & .84 & $2.05^{*}$ & .69 & .71 & 2.67 & 1.07 & 2.60 & .90 & .12 \\
\hline $\begin{array}{l}\text { Cardiovascular } \\
\text { Manifestations }\end{array}$ & 2.03 & .67 & 1.85 & .79 & .24 & 2.58 & 1.20 & 2.17 & .87 & .58 \\
\hline $\begin{array}{l}\text { Gastronomical } \\
\text { Manifestations }\end{array}$ & 1.64 & .94 & 1.76 & 1.12 & .26 & 1.78 & .91 & 1.67 & .80 & .17 \\
\hline Behavioral Manifestations & 1.50 & .54 & 1.52 & .49 & .04 & 1.81 & .89 & 1.67 & .82 & .25 \\
\hline MBI-ES total (0-6) & 2.93 & .82 & $2.58 *$ & .75 & .92 & 2.93 & .67 & 2.86 & .64 & .23 \\
\hline $\begin{array}{l}\text { MBI Emotional } \\
\text { Exhaustion }(0-6)\end{array}$ & 4.06 & 1.10 & $3.38 *$ & 1.22 & 1.21 & 3.81 & .94 & 3.48 & 1.19 & .40 \\
\hline $\begin{array}{c}\text { MBI Depersonalization (0- } \\
6)\end{array}$ & 2.09 & 1.03 & 1.93 & .74 & .24 & 2.25 & .66 & 2.17 & .76 & .15 \\
\hline $\begin{array}{c}\text { MBI Personal } \\
\text { Accomplishment (0-6) }\end{array}$ & 2.18 & 1.01 & 2.09 & .87 & .16 & 2.35 & .84 & 2.59 & .81 & .40 \\
\hline SA-45 GSI (45-225) & 73.36 & 21.41 & 68.36 & 19.08 & .34 & 76.92 & 18.85 & 71.08 & 14.02 & .43 \\
\hline SA-45 Depression & 1.83 & 1.05 & 1.41 & .58 & .71 & 1.70 & .83 & 1.45 & .51 & .51 \\
\hline SA-45 Anxiety & 1.85 & .63 & 1.65 & .32 & .30 & 1.83 & .60 & 1.63 & .41 & .30 \\
\hline
\end{tabular}

* Mean differences from pre- to post-intervention significant at $p<.05$. 
Note:

Lower scores on the:

TSI indicate less stress.

MBI-ES indicate fewer symptoms of burnout.

SA-45 indicate higher well-being.

Negative $d$ values indicate poorer outcomes. 
Table 3.7 Mean comparisons of teacher mindfulness by group.

\begin{tabular}{|c|c|c|c|c|c|c|c|c|c|c|}
\hline \multirow[b]{3}{*}{ Outcome measure (scale) } & \multicolumn{5}{|c|}{ Intervention $(n=11)$} & \multicolumn{5}{|c|}{ Waitlist-control $(n=12)$} \\
\hline & \multicolumn{2}{|c|}{ Pre } & \multicolumn{2}{|c|}{ Post } & \multirow[b]{2}{*}{$d$} & \multicolumn{2}{|c|}{ Pre } & \multicolumn{2}{|c|}{ Post } & \multirow[b]{2}{*}{$d$} \\
\hline & $M$ & $\mathrm{SD}$ & $M$ & $\mathrm{SD}$ & & $M$ & $\mathrm{SD}$ & $M$ & SD & \\
\hline FFMQ total $(1-5)$ & 3.32 & .43 & 3.47 & .39 & .56 & 3.37 & .46 & 3.44 & .51 & .23 \\
\hline FFMQ Observe & 3.33 & .51 & 3.43 & .46 & .33 & 3.22 & .85 & 3.06 & .87 & -.22 \\
\hline FFMQ Describe & 3.60 & .78 & $3.87 *$ & .64 & .76 & 3.91 & .47 & 3.98 & .57 & .20 \\
\hline $\begin{array}{c}\text { FFMQ Act with } \\
\text { Awareness }\end{array}$ & 3.11 & .56 & 3.31 & .47 & .51 & 3.33 & .50 & 3.41 & .66 & .20 \\
\hline FFMQ Nonjudgment & 3.31 & .89 & 3.39 & .99 & .16 & 3.26 & .87 & 3.54 & .84 & .41 \\
\hline FFMQ Non-reactivity & 3.25 & .38 & 3.31 & .47 & .20 & 3.11 & .74 & 3.19 & .68 & .20 \\
\hline
\end{tabular}

* Mean differences from pre- to post-intervention significant at $p<.05$.

Note:

Lower scores on the FFMQ indicate less mindfulness.

9 Negative $d$ values indicate poorer outcomes. 


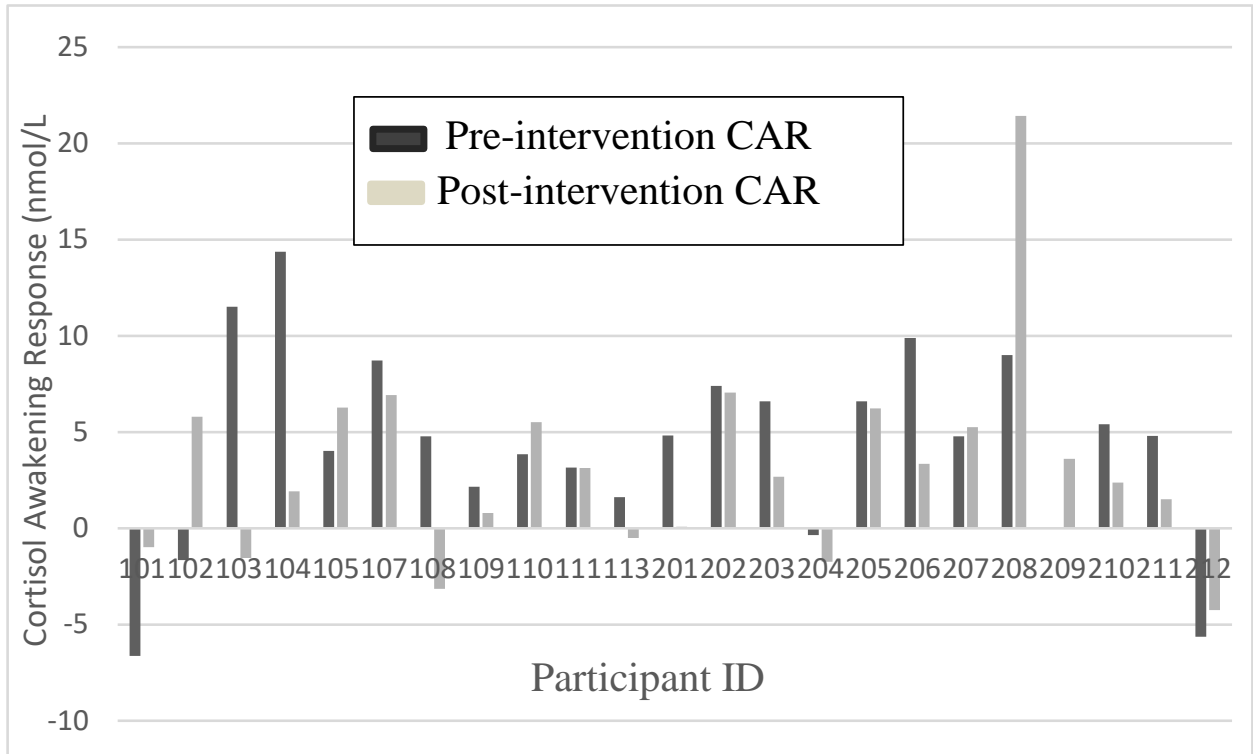

Figure 3.1 Participant cortisol awakening response (CAR) values at pre- and post-intervention. 


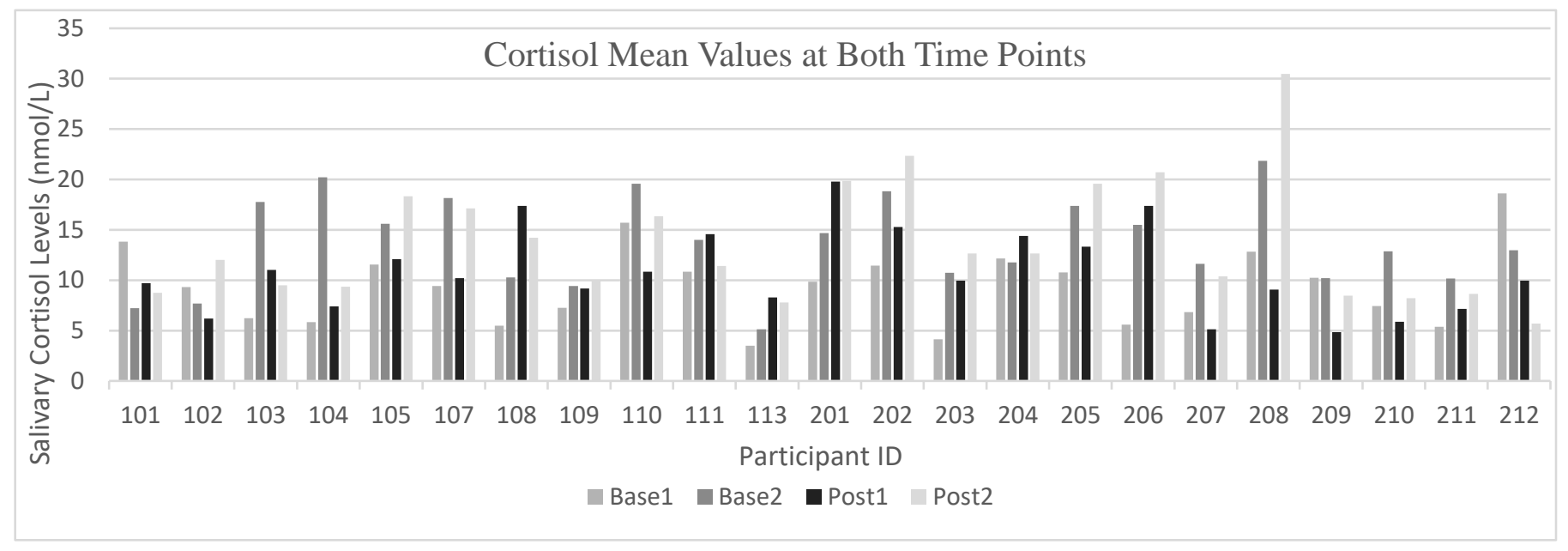

Figure 3.2 Participant salivary cortisol values at waking and 30 minutes post-waking. 


\section{CHAPTER 4}

\section{DISCUSSION}

The primary aim of this study was to test whether a brief mindfulness-based intervention (bMBI) was efficacious in reducing teacher stress, burnout, and psychological distress. The results of this randomized waitlist-control design indicate that only six total hours of direct face-to-face contact appears to be sufficient to significantly reduce some markers of stress and burnout in teachers. These findings provide insight into the minimally effective dose needed to target this at-risk population and optimize the cost-effectiveness of MBI interventions for teachers. The first part of our second aim was to investigate the extent to which the teachers engaging in a bMBI would improve across all dimensions of mindfulness compared to a waitlist-control group. Three of the five dimensions of mindfulness demonstrated some improvement with small-to-medium effect sizes (i.e., observe, act with awareness, and describe dimensions). One of these dimensions, the describe dimension of mindfulness, which involves the capacity to effectively utilize language to interpret the nature of one's experience, was significant, which suggests the intervention implemented in the current study has particularly strong effects on this dimension. Finally, we also investigated the extent to which changes in self-reported mindfulness from pre- to post-intervention impacted changes to teacher stress, burnout, and psychological distress in the intervention group to provide insight into the extent to which mindfulness and its separate domains functioned as a mechanism 
of change. Although a small sample size prevented relations from reaching significance, notable trends in the relations between changes in mindfulness and teacher outcomes suggest dimensions of mindfulness differentially impact teacher outcomes. These findings can inform future research endeavors seeking to develop and implement more cost-effective MBIs for teachers that aim to deduce their curriculum in a more targeted manner.

\subsection{Impact of the Intervention on Stress, Burnout and Psychological Distress}

Despite having little power to detect significant effects in the intervention group $(n=11)$, the current study demonstrated significant findings in the expected direction suggesting the bMBI has robust effects for reducing teachers' stress, burnout, and depression. Further examination of various components of stress indicate it was particularly effective for reducing stress related to time management, work, professional distress (e.g., "I am not progressing in my job as rapidly as I would like"), professional investment (e.g., "I am not emotionally/intellectually stimulated on the job"), and fatigue. Reductions in burnout were primarily related to emotional exhaustion (i.e., the initial phase of burnout in which teachers' capacity for coping with demands becomes overwhelmed). Unlike other MBI studies for teachers (see Table 1 for review), our study also corrected for multiple comparisons to account for family-wise error. Even after accounting for these corrections using the Holm-Bonferroni Method, significant reductions in teacher stress, time management, work-related stress, and emotional exhaustion were sustained suggesting the intervention was particularly impactful for these outcomes. In further support of this notion, the effect sizes (ranging from $d=1.03$ to 1.54) for these significant effects are equivalent to (e.g., Beshai et al., 2016) or far 
exceed those found for markers of stress and burnout in other MBI studies (see Table 1) despite many of these studies implementing interventions of longer duration that required a greater amount of resources (e.g., Flook et al., 2013 [9 sessions with 26 direct contact hours]; Roeser et al., 2013 [11 sessions with 30 direct contact hours]). Even many of the effect sizes across non-statistically significant measures of stress and burnout were similar to those seen in these studies of far greater duration, which suggests an intervention of shorter duration (i.e., four sessions and six face-to-face contact hours) can be at least as effective, if not more effective, as those of longer durations that require far more resources and time investment from teachers. Furthermore, the only existing MBI study in the literature of shorter duration than the current study (Ancona \& Mendleson, 2014; six sessions and four-and-a-half face-to-face contact hours) did not demonstrate a significant effect for stress or burnout and demonstrated only small-to-medium effect sizes for these constructs (i.e., $d=.54$ and $d=.43$ for the TSI and Emotional Exhaustion component of the MBI-ES, respectively). This suggests that the additional time (i.e., 90 minutes) in the bMBI curriculum used in the current study may have provided meaningful incremental differences that have clinical significance.

The current study was only the third in the literature (Flook et al., 2013; Roeser et al., 2013) to demonstrate positive significant changes for symptoms of teacher burnout. Flook and colleagues (2013) identified significant changes for two out of three components of burnout (i.e., emotional exhaustion and personal accomplishment). Roeser and colleagues (2013) found a significant change on a measure that more broadly encompassed all three components of burnout. Although the current study only identified significant changes for the emotional exhaustion component of burnout after controlling 
for multiple comparisons, the effect sizes of the current study for measures of emotional exhaustion (i.e., $d=1.21$ ) and burnout more broadly (i.e., $d=.99$ ) exceed those demonstrated in Flook and colleagues' study $(2013 ; d=.25$ for the emotional exhaustion component) and Roeser and colleagues' study (2013; $d=.76$ for burnout more broadly). These findings are particularly important as they suggest that the effects of the intervention were at least as strong for the bMBI implemented in the current study despite the abbreviations in dosage compared to other studies demonstrating significant effects for burnout (i.e., six total contact hours in comparison to 26 direct contact hours for Flook et al., 2013 and 30 direct contact hours for Roeser al., 2013).

The current study also aimed to address areas of methodological rigor (i.e., utilizing a randomized waitlist-control design and assessing for physiological markers of stress) lacking in many of the MBI studies for teachers in the literature (see Table 1). Physiological markers of stress, such as the cortisol awakening response (CAR), are vital in examining the effects of chronic stress to the biological systems of individuals (e.g., allostatic load; McEwen, 1998), which are not captured by self-report measures. The current study identified a small effect (i.e., Cramer's $V=.194$ ) whereby an adaptive shift occurred in physiological indicators of stress (as measured by CAR) for those in the intervention group. This was contrary to the maladaptive shift from baseline to post found in the control group for which a medium effect was observed (i.e., Cramer's $V=.354$ ). This finding is critical as it aligns with two previous studies (Harris et al., 2016; Flook et al., 2013) that suggest a portion of teachers who do not receive appropriate interventions to effectively manage stress typically experience a maladaptive shift in physiological functioning during the course of an academic semester. Despite these observed trends, we 
did not find significant improvements in physiological indicators of stress as measured by CAR. As previously discussed, this may be due to teachers presenting with variable degrees of stress and burnout producing differing physiological patterns; this also may be due to the heterogeneity in the CAR at a population level that makes the detection of statistically significant trends difficult in small samples (Wust et al., 2000; Fries et al., 2009; Stalder et al., 2016). The former notion is supported through closer interpretation of the participants' cortisol values at waking and 30 minutes post-waking at baseline and post-intervention (see Figures 3.1 and 3.2). These figures demonstrate that some teachers with a maladaptive CAR exhibited a heightened response (i.e., increases above $75 \%$ of post-waking values) and others exhibited blunted responses (i.e., increases below $38 \%$ of post-waking values). Although both of these responses are maladaptive, the field's understanding of the pattern and progression of these responses across different populations and contexts is still in an early developmental stage. However, drawing from this extant literature may help to provide meaningful interpretations regarding the wide variations in physiological responses among teachers in the current study. In particular, Miller and colleagues' (2007) large systematic review of studies that examined CAR across individuals experiencing a variety of contextual factors showed that those facing chronic social stressors or in caregiving roles, which is typical for teachers, may demonstrate higher morning cortisol levels, whereas those experiencing chronic physical forms of stress (e.g., threats to life such as violence or poverty) tend to demonstrate blunted responses to stress (i.e., fall below the adaptive range). However, additional evidence from an earlier study suggests that social stress that is similar in nature to that which is experienced by those in caregiving roles (i.e., teachers) and sufficiently chronic, 
may still eventually lead to more severe symptoms of burnout and the development of a blunted response (Flook et al., 2013; Harris et al., 2016; Pruessner et al., 1999). For the sample included in the current study, we identified both types of maladaptive CAR profiles (i.e., heightened and blunted) suggesting teachers have varying physiological responses to their experiences of stress.

Furthermore, Moya-Albiol and colleagues (2010) noted there may be salient distinctions between the dimensions of burnout that may not be fully recognized when collapsing these different dimensions into a singular index for burnout, and thus, stressed the importance of examining the components of burnout (i.e., emotional exhaustion, depersonalization, and personal accomplishment) separately when examining this construct's relation to physiological markers of stress. However, no existing studies have examined the CAR in relation to all of these separate dimensions. One study (Sonnenschein et al., 2007) identified a blunted CAR for those high in self-reported symptoms of emotional exhaustion, but this study did not include the other dimensions of burnout in their investigation. Research shows that symptoms of emotional exhaustion occur prior to the other stages of burnout (Maslach, Schaufeli, \& Leiter 2001), and the corresponding subscale is typically the first to show signs of burnout (Leiter, 1993). In the current study, the means of each respective subscale for the teachers in the intervention group at baseline (Emotional Exhaustion mean $=4.06, \mathrm{SD}=1.04$ range $=$ 4.33; Depersonalization mean $=2.09, \mathrm{SD}=1.03$ range $=2.80 ;$ Personal Accomplishment $=2.18, \mathrm{SD}=1.01$ range $=3.25)$, suggests that, on average, teachers in our sample were experiencing severe symptoms of emotional exhaustion but were not as high in the other dimensions. The relation between a blunted cortisol response and emotional exhaustion 
identified by Sonnenschein and colleagues (2007), was supported in our study as results indicate that there were more teachers with a blunted response at baseline than a heightened response (i.e., six teachers compared to four). However, there were seven teachers who continued to have a blunted response despite significant decreases in symptoms of emotional exhaustion observed post-intervention, providing some contradiction to Sonnenschein's assertion and suggesting a need for further exploration. Given the lack of change on other dimensions of burnout (i.e., depersonalization and personal accomplishment) in the current study, perhaps it is not until the symptoms of these dimensions of burnout subside that the CAR would return to an adaptive range. However, the dearth of literature in this area makes it difficult to determine the nature of the CAR across dimensions of burnout.

Alternatively, one study identified a lack of association between burnout and physiological stress systems (Langelaan, Schaufeli, van Doornen, Bakker, \& van Rhenen, 2007) suggesting that the CAR may be implicated by teacher stress and unrelated to burnout. The significant relation between changes in CAR and changes in self-reported symptoms of teacher stress (represented by the full scale TSI) in the current study suggests that the CAR was more sensitive to changes in teacher stress throughout the course of the intervention than it was for burnout. Additionally, we found a decrease in teachers demonstrating a heightened response from pre-to post-intervention (i.e., from three to one), and little relation to those in the blunted response associated with burnout. Therefore, the findings in the current study may be in support of Miller and colleagues (2007) findings that shows those with high amounts of social stress demonstrate a higher CAR. 
Finally, it is also possible that the effects of burnout on the CAR were masked by teacher stress. Although the CAR is a generally stable physiological marker that accounts for more extended experiences of individuals, it has been demonstrated that the CAR can be sensitive to next-day anticipatory stress (Fries et al., 2009). This suggests it is possible that some of the teachers' heightened responses had declined by post-intervention due to their increased management of these day-to-day stressors, but some then began to demonstrate a blunted CAR since many physiological systems associated with increased allostatic load (particularly those in women) can take longer to recover (Gustafsson, Janlert, Theorell, Westerlund, \& Hammarström, 2011). That is, some teachers who were experiencing severe symptoms of emotional exhaustion at baseline may have been exhibiting a heightened response as opposed to a blunted response due to simultaneous heightened anticipatory social stress they had been experiencing on a day-to-day basis. However, after learning to better manage this stress, their dysfunctional CAR profile may have been more consistent with that which would be expected of someone who had experienced severe symptoms of burnout (i.e., a blunted response) but had not yet recovered in full physiologically. Regardless, the trends observed in the current study suggest that the bMBI was effective in shifting individuals from maladaptive (whether it was heightened or blunted) to adaptive physiological functioning with regards to the CAR. Furthermore, the negative shift towards more maladaptive responses for teachers in the control group (with three of these teachers moving from adaptive to blunted) suggests that the bMBI may also be protective against the deteriorating physiological effects of chronic stress and burnout that is experienced by teachers throughout the course of a 
typical semester. It is important for future studies to continue to include markers of physiological stress to validate these findings in larger and more diverse samples.

The current study also assessed the impact of the intervention on changes from pre- to post-intervention on general psychological symptoms, anxiety, and depression. Of these, findings suggest that the intervention has potential for reducing symptoms of depression. The medium effect size for reductions in depression for the intervention group was substantially larger than the small effect for anxiety and suggests there was a larger impact on symptoms of depression as opposed to anxiety. There are a number of reasons why the bMBI may have been more impactful for symptoms of depression than other psychological symptoms. Multiple studies have demonstrated a rapid decline in reported symptoms of depression following brief interventions (i.e., ranging from one-tosix sessions measured over the course of two-to-six weeks) aimed at increasing engagement in behaviors that align with an individual's values (Gawrysiak, Nicholas, \& Hopko, 2009; Kohtala, Lappalainen, Savonen, Timo, \& Tolvanen, 2015; Kyllönen et al., 2018). Given the emphasis on self-regulation and intentionality in the current study, it is unsurprising that there was a similar impact as interventions that more directly target depression. However, many mindfulness-based strategies work mechanistically by first drawing one's nonjudgmental awareness to the psychological and physiological experience of emotions, which allows for a greater attentional capacity and sustained engagement during uncomfortable emotional experiences as opposed to engaging in experiential avoidance (i.e., the avoidance of internal experiences such as thoughts, feelings, and emotions; Hayes, Wilson, Gifford, Follette, \& Strosahl, 1996). Although this decrease in experiential avoidance lends itself to improving symptoms of depression 
(via engagement in values-based behaviors that alleviates these symptoms), it may temporarily increase one's awareness and thus the intensity of acute anxiety before it begins to decline as a result of prolonged exposure to the anxiety-provoking events and contexts (Abramowitz, Deacon, \& Whiteside, 2019; Mitmansgruber, Beck, Höfer, \& Schüßler, 2009). Although there were small effect sizes for improvements in symptoms of anxiety for the intervention group, it is possible these effects would be larger with the increased exposure that could result from more practice and mastery in attending to the present moment during anxiety-provoking situations over time.

\subsection{Mindfulness as Mechanisms of Change}

Although small-to-medium effect sizes were observed for improvements on three out of five mindfulness dimensions from pre- to post-intervention in the intervention group, only the change in the describe dimension was statistically significant, which suggests there was a particularly robust change in this dimension (see Table 10). The item content of the subscale representing the describe dimension (e.g., "When I have a sensation in my body, it's difficult for me to describe it because I can't find the right words") demonstrates an ability to utilize language processes to better capture the phenomenological experiences of the individual. It is likely these changes were driven by both didactic and discussion-based elements of the intervention as these generally targeted the operationalization of the components of mindfulness and provided teachers with exposure to other teachers' descriptions of their experiences. The intervention's emphasis on increasing awareness of how to more effectively self-regulate stress management behaviors was reflected in the considerable (i.e., a medium effect size) improvements to the acting with awareness dimension of mindfulness, which differs from 
past studies that assessed these separate dimensions of mindfulness (Jennings et al., 2013; Flook et al., 2013; Harris et al., 2016). Furthermore, to allow for more session time to be devoted to applications to self-regulation, the intervention included substantially less engagement in mindfulness practice during sessions than other existing MBIs for teachers (i.e., approximately $10-15 \%$ of session time compared to other studies that had sessions comprised of up to $50 \%$ of time spent in mindfulness practice; e.g., Jennings et al., 2013; Harris et al., 2016; Roeser et al., 2013; Flook et al., 2013), however the small effect size demonstrated on the observe dimension of mindfulness indicates that there were still meaningful improvements on dimensions that are highly associated with this practice. Although statistical significance was not met on all dimensions, observed improvements on the full scale of mindfulness and these three separate dimensions suggest that the intervention was functional in improving targeted mechanisms of change.

To determine whether intervention outcomes were related to improvements in these targeted mindfulness components, the current study examined the degree to which changes in dimensions of mindfulness correlated with changes in intervention outcomes. Our findings demonstrate that there were moderate or strong correlations between at least two or more dimensions of mindfulness with each of the self-reported teacher outcomes (i.e., the TSI, MBI-ES, and GSI of the SA-45). Although no significant correlations were observed due to utilizing a small sample, these findings demonstrate relations that can help facilitate the development of MBI curriculum in future studies seeking to identify the particular mechanisms that differentially target intervention outcomes. Of these dimensions, the describe dimension showed a particularly strong correlation with selfreported teacher stress and was the only dimension to demonstrate even a small 
correlation with CAR (see Table 11). This finding provides further evidence to support the importance of retaining didactic and discussion-based elements for MBIs seeking to reduce teachers' stress. Given each of the dimensions of mindfulness (i.e., observe, describe, act with awareness, nonjudgment, non-reactivity) demonstrated at least one moderate relation with a self-reported outcome measure, these findings suggest each dimension of mindfulness plays a critical role in decreasing elements of teacher stress and burnout and improving general psychological functioning. Specifically, it appears the describe and act with awareness dimensions of mindfulness were the most impactful in reducing teacher stress and burnout (as measured by the TSI and MBI-ES), while the observe and non-reactivity dimensions were most highly related to changes in general psychological distress (as measured by the SA-45).

Findings related to burnout are particularly informative given only two other studies demonstrated significant changes for burnout. As expected, it appears including elements of group-based discussion and an emphasis on self-regulation (those which are most closely conceptually linked to the describe and act with awareness dimensions of mindfulness, respectively) are imperative for studies seeking to decrease teacher burnout. However, the greater strength in relations between the observe and non-reactivity dimensions (i.e., moderate-to-strong) of mindfulness with psychological distress suggests that MBIs targeting teachers who experience more broad-ranging and severe psychological symptoms may see increased benefit from interventions that focus on developing the skill of simply observing one's experience and mitigating the maladaptive behavioral responses they may have already developed. The current study is the first to examine these relations between the separate dimensions of mindfulness and teacher 
outcomes in the context of an MBI, and the findings demonstrate that the dimensions have unique influences on teacher outcomes. These findings can be used to help tailor interventions to more effectively meet the needs of teachers who may present with different concerns across communities and districts. For instance, it may be that a subgroup of teachers is struggling with more severe psychological symptomology in a particular area and would benefit more from a tailored design that emphasized elements of observe and non-reactivity dimensions of mindfulness in place of other components. Future studies should seek to build on this work using larger and more diverse samples to help guide this tailoring of interventions moving forward so that future interventions can continue to employ briefer designs that are more cost-effective.

Aside from the describe dimension of mindfulness, the changes across dimensions of mindfulness from pre- to post-intervention were not statistically significant and may have contributed to a lack of significant relations between these dimensions and teacher outcomes. One reason that only small-to-medium changes were observed on these dimensions may relate to the typical progression of mindfulness skills throughout MBIs and with continued practice. The general trends in the literature suggest that participants new to mindfulness tend to overestimate their mindfulness skills at baseline and will rate themselves lower on measures of mindfulness during the early stages of skill development after gaining some structured exposure to these (Roemer \& Orsillo, 2003). This research shows that self-efficacy for the skill grows only after extended and consistent practice. It has also been posited that mindfulness skills compound overtime and individuals do not feel as though they have gained a sense of mastery until they have developed an extensive practice that allows them to integrate mindfulness with 
complementary skill sets that facilitate effective stress management and effectively generalize this across contexts (Baer, 2003). Although the bMBI did not demonstrate large effects on the dimensions of mindfulness, it is possible that the degree of changes in these dimensions were sufficient to produce the large improvements on teacher outcomes (i.e., even small changes in mindfulness skills may produce large effects).

However, it is also possible that there may be other key mechanisms that accounted for some of the positive intervention effects that the current study did not directly measure. For instance, there is an abundance of research that shows teacher self-efficacy, a teacher's beliefs in his or her capacity to execute behavioral patterns in teachingspecific domains that are needed to perform competently as a professional (Bandura, 1997), mitigates stress, burnout, and general psychological distress (Schwarzer \& Hallum, 2008; Skaalvik \& Skaalvik, 2016). Although the current study did not directly measure this construct, the TSI contains item content that aligns with some elements of teachers' self-efficacy. In particular, the time management component of teacher stress (measured using the Time Management subscale of the TSI in the current study) includes multiple items specific to teachers' belief in their capacity to effectively plan their schedule and competently engaging in vocationally-specific tasks despite feeling time constraints (e.g., "I think about unrelated matters during conversations" and "I rush in my speech"). There was a statistically significant and large effect on this subscale from preto post-intervention in the intervention group, suggesting that the bMBI impacted this domain of functioning. Given the conceptual overlap between this component of stress and teacher self-efficacy, and the previous research demonstrating the inverse relation between teacher self-efficacy and teacher psychological distress, it is possible that 
increases to teacher self-efficacy (which were not directly measured in the current study) may have contributed to some of the positive effects seen across various teacher outcome variables. Furthermore, it is possible that time management and other components of teacher stress (e.g., discipline and motivation, professional investment, fatigue manifestations, etc.) may function such that improvements over the course of the intervention facilitate improvements in mindfulness, which can in turn function as a synergistic positive feedback loop. Although this process is difficult to capture statistically without follow-up measures and larger sample sizes, it is important to note that stress is additive and the resources required to cope with increasing demands are compounding (Derogatis, 1987).

Another possibility for an unaccounted mechanism of change stems from evidence in a separate study from our research group (Roberts et al., under review) that collected qualitative information on the participants of the bMBI in the current study. These participants described that elements of peer social support (e.g., "I think that part of the effectiveness is sharing/hearing others") innate to group-based interventions, such as the bMBI in the current study, may have contributed to some of the positive intervention effects. Past research demonstrates an association between both teacher stress (Griffith, Steptoe, \& Cropley, 1999) and burnout (Burke, Greenglass, \& Schwarzer, 1996; Greenglass et al., 1996), and some MBIs (Reiser et al., 2016; Reiser \& McCarthy, 2018) integrated specific social support elements (e.g., allotting time for group discussion regarding shared stressors and ways to support one another in addressing stressors outside the sessions) into their curriculum to target this mechanism. These studies did not demonstrate significant improvements to markers of stress, but that does not entirely rule 
out the possibility that this mechanism was implicated in the current study as there again was not a quantitative measure of this construct. Without direct measurement, the extent to which social support functioned as a mechanism of change in the current intervention remains unclear.

One final possibility is that compassion could have accounted for some of the changes in outcome variables. There are a number of studies linking increases in compassion of the self and others to better psychological functioning in teachers (e.g., Jennings, 2015; Roeser et al., 2013; Beshai et al., 2016). There are also strong conceptual links between mindfulness and compassion (Renshaw \& O’Malley, 2014) with early conceptualizations of mindfulness indicating compassion as a primary intention and necessary component for the cultivation of mindfulness skills (Shapiro et al., 2006). Given the intervention was aimed primarily at stress management, the intervention curriculum in the current study encouraged teachers to explore their values related to stress management when discussing teachers' intentions for their cultivation of mindfulness. However, it is possible that changes to teachers' compassion for self and others also accounted for some of the variance of the change in stress, burnout, and psychological distress given these values underlie goals related to reducing stress.

\subsection{Limitations and Future Directions}

Despite employing elements of methodological rigor that were missing from many previous studies (i.e., randomized control design and measurement of physiological stress), the current study still found statistically significant positive intervention effects suggesting that the bMBI used in the current study had a particularly strong impact. 
However, the small sample (i.e., 24 teachers from a local high school) recruited for the current study may have contributed to only four outcome variables demonstrating statistically significant changes from pre- to post-intervention after correcting for familywise error. This notion is supported as two other outcomes demonstrated large effect sizes (i.e., the Professional Distress subscale of the TSI and the MBI-ES full scale) and four demonstrated medium effect sizes (i.e., the Professional Investment, Emotional Manifestations, and Fatigue Manifestations subscales of the TSI, and the Depression subscale of the SA-45), which are all likely to reach statistical significance in larger samples. Our sample was largely homogenous in terms of race ( $91.3 \%$ white), education (95.7\% having a Masters or Doctoral degree), and years of teaching experience (60.8\% of teachers having over 10 years of experience; see Table 3), and these protective factors are likely to have made teachers more resilient than the average teacher. Furthermore, the current high school from which the teachers were sampled is a high-achieving school who are likely to face a different set of stressors (albeit, not necessarily more or less severe in nature) than teachers in low income areas with students who reside in underserved communities. Future studies should aim to replicate these findings in a larger and more diverse sample of teachers to explore what types of stressors teachers face across different grade levels and at schools with varying resources.

The current study obtained certification for the bMBI to count toward annual CEC requirements for teachers who participated in the bMBI effectively creating a natural active control whereby a comparison could be made to those teachers who did not participate in the bMBI and instead engaged in the typical CEC programs offered to teachers. Although this increased the rigor of the study design, there were other 
limitations that future studies should seek to address. For instance, teachers who enrolled in the study were interested in both stress management and mindfulness, thus it is possible they chose to engage in other CEC opportunities relating to these topics in order to more effectively build these skills. The small improvements in mindfulness skills observed from pre- to post-intervention in the waitlist-control group would support this notion. To protect against this, future studies should seek to design a standardized CEC offered to control group teachers that can be used as a comparison for the intervention group and reduces the chances that teachers in the control group seek out other similar opportunities that may implicate similar mechanisms. Given the strong associations with social support and teacher stress described above (Burke et al., 1996; Greenglass et al., 1996), and the positive qualitative findings relating to social support identified for the intervention in the current study that are described in a separate study (Roberts et al., under review), it may be useful to utilize a social support intervention as the active control to help determine the extent to which mindfulness functions as a mechanism of change beyond the effects of social support.

It is also possible that participants from the intervention and waitlist-control groups being chosen from the same school may have produced contamination effects whereby mindfulness skills taught to the intervention group either intentionally or unintentionally shared information from the bMBI with their colleagues that were not a part of that group. Although there were no significant changes in teacher outcomes from pre- to post-intervention for the control group, some small improvements were observed in stress and burnout, and slightly larger improvements (i.e., medium effect sizes) were observed in depression and cardiovascular manifestations of stress (see Table 9). 
However, these changes may also be representative of the changing psychological wellbeing of staff towards the end of the semester (i.e., higher demands and a greater number of stressors at the beginning of the semester as opposed to the lower demands and fewer stressors at the end of the semester). The observed maladaptive shift in CAR for the control group contradicts this notion, but it is possible that this is due to the delay in change that is often observed in physiological stress systems after exposure to chronic stressors (Gustafsson et al., 2011). Although the randomized control design allows us to tease apart some possible spillover effects of positive changes, the only way to fully protect against this in future studies is to utilize a stratified sampling method whereby teachers selected to participate in the intervention have no opportunity to interact with those in the control group.

Future studies can also seek to build off strengths in the methodological rigor of the current study by collecting measures of teachers' engagement in mindfulness practice outside of sessions and follow-up measurement on teachers following the cessation of the intervention. Past studies have shown a wide variability in the amount of time teachers spend in mindfulness practice outside of session (Benn et al., 2012; Harris et al., 2016; Roeser et al., 2013). The current study did not collect a measure of engagement outside of sessions, but future research would benefit from teasing apart differences in acquisition of mindfulness skills amongst teachers based on practice outside of session given the association between practice of mindfulness skills and the acquisition of the skills (Reomer \& Orsillo, 2003). Collecting follow-up measures would help to determine whether the positive improvements of the intervention were sustained in the months following the intervention, thereby making a more significant impact. Furthermore, the 
research supporting that the positive impacts of mindfulness may not be fully observed until an individual has extensively practiced the skills (Baer, 2003) would suggest it may be possible for teachers to experience further benefits of the bMBI that are left undocumented. Studies employing follow-up measurement may also be able to test the differences in ways to continue promoting practice and engagement in mindfulness after the cessation of the intervention. These findings can help determine how to best sustain teachers' mindfulness practice.

The findings and observations of the bMBI alluded to other ways that future studies may be able to enhance the positive intervention effects observed in the current study. For instance, the qualitative components of another study conducted on this intervention show the importance of social support amongst the teachers (Roberts et al., in preparation). This suggests it may be useful to cultivate more interactions both in-andoutside of the session to further promote positive changes. Furthermore, given the association between practice time and the development of mindfulness skills (Reomer $\&$ Orsillo, 2003) it would be useful for future studies to identify ways to continue to encourage practice outside of sessions. The current study provided opportunities and encouragement for engagement in mindfulness practice outside of session with a list of mindfulness exercises in the appendix of their workbooks, space to reflect on practice and implementation of skills in daily life, and group-discussion at the outset of sessions two through four. During discussions in session, teachers noted that they would be likely to practice more if they had more reminders and a system to remain accountable in between sessions. Some solutions to this problem include having intervention facilitators send more reminders, identifying a phone app that can remind teachers to practice and monitor 
their behaviors, and/or providing teachers with a partner or a group of partners who can increase teachers' accountability for practicing outside of sessions. Adding any of these components in future MBI studies for teachers could improve outcomes without a need for additional resources (making it a cost-effective addition) and allow for teachers to get the added benefits of more practice time in a manner that they can flexibly incorporate into their busy schedules. The latter suggestion (i.e., giving teachers a partner or group to provide reminders and accountability outside of session) may have a particularly strong effect as it can also allow teachers to capitalize on the positive benefits of social support that were reported in qualitative findings for the bMBI (Roberts et al., under review).

\subsection{Conclusion}

The current study implemented and tested a brief mindfulness-based intervention (bMBI) to reduce teachers' stress and burnout using a randomized waitlist-control design. There were several significant improvements for self-reported teacher stress, burnout, and psychological distress in the intervention group but not in the control group. Although trends suggest there may have also been observed improvements in the cortisol awakening response (CAR) for the intervention group and deteriorating effects for the control group, future research should seek to replicate findings in a larger sample in order to utilize analyses that are better able to tease apart the nuances in participants' physiological functioning. Additionally, the current study did not have sufficient power to determine whether the separate dimensions of mindfulness served as mechanisms of change despite observing small-to-medium effects on these variables. Future research can use the findings from the current study to guide the development of bMBIs and can further expand our understanding of the mechanisms of change in bMBIs by examining 
these dimensions of mindfulness in larger samples, collecting follow-up measurement, and exploring other potential variables that may contribute to the positive intervention outcomes (e.g., social support, teacher self-efficacy, and compassion). The current study is vital in guiding future studies that aim to address the critical need to reduce teachers' stress and burnout in a cost-effective manner. 


\section{REFERENCES}

Abdi, H. (2010). Holm's sequential Bonferroni procedure. Encyclopedia of research design, 1(8), 1-8.

Abramowitz, J. S., Deacon, B. J., \& Whiteside, S. P. (2019). Exposure therapy for anxiety: Principles and practice. Guilford Publications.

Adam, E. K., \& Gunnar, M. R. (2001). Relationship functioning and home and work demands predict individual differences in diurnal cortisol patterns in women. Psychoneuroendocrinology, 26(2), 189-208.

Aickin, M., \& Gensler, H. (1996). Adjusting for multiple testing when reporting research results: the Bonferroni vs Holm methods. American Journal of Public Health, 86(5), 726-728.

Al-Fudail, M., \& Mellar, H. (2008). Investigating teacher stress when using technology. Computers and Education, 51, 1103-1110.

Alliance for Excellent Education. (2004). Tapping the Potential: Retaining and Developing High Quality New Teachers. Retrieved from https://all4ed.org/reports-factsheets/tapping-the-potential-retaining-anddeveloping-high-quality-new-teachers/

Alliance for Excellent Education. (2005). Teacher attrition: A costly loss to the nation and to the 
states. Retrieved from http://www.all4ed.org/publications/TeacherAttrition.pdf

Ancona, M.R., \& Mendelson, T. (2014). Feasibility and preliminary outcomes of a yoga and mindfulness intervention for school teachers. Advances in School Mental Health Promotion, 7(3), 156-170.

Aspinwall, L. G., \& Taylor, S. E. (1997). A stitch in time: Self-regulation and proactive coping. Psychological bulletin, 121(3), 417.

Auerbach, R. P., Mortier, P., Bruffaerts, R., Alonso, J., Benjet, C., Cuijpers, P., ... \& Murray, E. (2018). WHO World Mental Health Surveys International College Student Project: prevalence and distribution of mental disorders. Journal of Abnormal Psychology, 127(7), 623.

Awa, W. L., Plaumann, M., \& Walter, U. (2010). Burnout prevention: A review of intervention programs. Patient education and counseling, 78(2), 184-190.

Baer, R. A., Smith, G. T., Hopkins, J., Krietemeyer, J., \& Toney, L. (2006). Using selfreport assessment methods to explore facets of mindfulness. Assessment, 13(1), $27-45$.

Baer, R. A., Smith, G. T., Lykins, E., Button, D., Krietemeyer, J., Sauer, S., ... \& Williams, J. M. G. (2008). Construct validity of the five-facet mindfulness questionnaire in meditating and nonmeditating samples. Assessment, 15(3), 329342.

Bandura, A. (1991). Social cognitive theory of self-regulation. Organizational Behavior and Human Decision Processes, 50(2), 248-287. 
Bandura, A. (2005). The primacy of self-regulation in health promotion. Applied Psychology, 54(2), 245-254.

Bandura, A., Caprara, G. V., Barbaranelli, C., Gerbino, M., \& Pastorelli, C. (2003). Role of affective self-regulatory efficacy in diverse spheres of psychosocial functioning. Child development, 74(3), 769-782.

Bandura, A., Freeman, W. H., \& Lightsey, R. (1999). Self-efficacy: The exercise of control.

Bellingrath, S., \& Kudielka, B. M. (2017). Biological pathways to stress-related disease vulnerability in educators. In T. M. McIntyre, S. E. McIntyre, D. J. Francis, T. M. McIntyre, S. E. McIntyre, D. J. Francis (Eds.), Educator stress: An occupational health perspective (pp. 77-100). Cham, Switzerland: Springer International Publishing. doi:10.1007/978-3-319-53053-6_4

Benn, R., Akiva, T., Arel, S., Roeser, R.W. (2012). Mindfulness training effects for parents and educators of children with special needs. Developmental Psychology, 48(5), 1476.

Beshai, S., McAlpine, L., Weare, K., Kuyken, W. (2016). A non-randomized feasibility trial assessing the efficacy of a mindfulness-based intervention for teachers to reduce stress and improve well-being. Mindfulness, 7(1), 198-208.

Betoret, F.D. (2006). Stressors, self-efficacy, coping resources, and burnout among secondary school teachers in Spain. Educational Psychology, 26, 519-539. 
Borg, M., \& Riding, R.J. (1991). Stress in teaching: a study of occupational stress and its determinants, job satisfaction and career commitment among primary schoolteachers. Educational Psychology, 11, 59.

Boyle, G.J., Borg, M.G., Falzon, J.M., \& Baglioni, A.J., Jr. (1995). A structural model of the dimensions of teacher stress. British Journal of Educational Psychology, 65, 49-67.

Briner, R., \& Dewberry, C. (2007). Staff well-being is key to school success. London: Worklife Support Ltd/Hamilton House.

Brouwers, A., \& Tomic, W. (2000). A longitudinal study of teacher burnout and perceived self-efficacy in classroom management. Teaching and Teacher education, 16(2), 239-253.

Brown, K.W., Ryan, R.M., \& Creswell, J.D. (2007). Mindfulness: Theoretical foundations and evidence for is salutary effects. Psychological Inquiry, 18, 211237.

Burke, R. J., Greenglass, E. R., \& Schwarzer, R. (1996). Predicting teacher burnout over time: Effects of work stress, social support, and self-doubts on burnout and its consequences. Anxiety, stress, and coping, 9(3), 261-275.

CANTAB. Cambridge Cognition: CANTAB for Windows. Cambridge Cognition Limited; Cambridge, UK: 1999.

Carmody, J., \& Baer, R.A. (2008). Relationships between mindfulness practice and levels of mindfulness, medical and psychological symptoms and well-being in a 
mindfulness- based stress reduction program. Journal of Behavioral Medicine, 31, 23-33.

Chambless, D. L., \& Hollon, S. D. (1998). Defining empirically supported therapies. Journal of Consulting and Clinical Psychology, 66(1), 7.

Chida, Y., \& Steptoe, A. (2009). Cortisol awakening response and psychosocial factors: a systematic review and meta-analysis. Biological Psychology, 80(3), 265-278.

Chiesa, A., \& Serretti, A. (2009). Mindfulness-based stress reduction for stress management in healthy people: A review and meta-analysis. The Journal of Alternative and Complementary Medicine, 15(5), 593-600.

Cohen, J. (2013). Statistical power analysis for the behavioral sciences. Academic press.

Cohen, S., Janicki-Deverts, D., \& Miller, G. E. (2007). Psychological stress and disease. JAMA: Journal of the American Medical Association, 298(14), 1685-1687.

Cohen, S., Kessler, R. C., \& Gordon, L. U. (Eds.). (1997). Measuring stress: A guide for health and social scientists. Oxford University Press on Demand.

Collie, R.J., Shapka, J.D., \& Perry, N.E. (2012). School climate and social-emotional learning: Predicting teacher stress, job satisfaction, and teaching efficacy. Journal of Educational Psychology, 104(4), 1189-1204.

Cullen, M. (2011). Mindfulness-based interventions: An emerging phenomenon. Mindfulness, 2, 186-193. 
Davison, M. L., Bershadsky, B., Bieber, J., Silversmith, D., Maruish, M. E., \& Kane, R. L. (1997). Development of a brief, multidimensional, self-report instrument for treatment outcomes assessment in psychiatric settings: Preliminary findings. Assessment, 4(3), 259-276.

Dedovic, K., \& Ngiam, J. (2015). The cortisol awakening response and major depression: examining the evidence. Neuropsychiatric disease and treatment, 11, 1181-1189. doi:10.2147/NDT.S62289

Derogatis, L. R. (1987). The Derogatis stress profile (DSP): Quantification of psychological stress. In Research paradigms in psychosomatic medicine (Vol. 17, pp. 30-54). Karger Publishers.

De Vente, W., Olff, M., Van Amsterdam, J. G. C., Kamphuis, J. H., \& Emmelkamp, P. M. G. (2003). Physiological differences between burnout patients and healthy controls: blood pressure, heart rate, and cortisol responses. Occupational and Environmental Medicine, 60(suppl 1), i54-i61.

DeVol, R., Bedroussian, A., Charuworn, A., Chatterjee, A., Kim, I. K., Kim, S., \& Klowden, K. (2007). An unhealthy America: The economic burden of chronic disease.

Dunham, J., \& Varma, V. (1998). Stress in teachers: Past, present and future. London: Whurr. 
Emerson, L. M., Leyland, A., Hudson, K., Rowse, G., Hanley, P., \& Hugh-Jones, S. (2017). Teaching mindfulness to teachers: A systematic review and narrative synthesis. Mindfulness, 8(5), 1136-1149.

English, I., \& Campbell, D. G. (2019). Prevalence and characteristics of universal depression screening in US college health centers. Families, Systems, \& Health, 37(2), 131-149. https://doi-org.pallas2.tcl.sc.edu/10.1037/fsh0000411

Escuriex, B.F., \& Labbé, E.E. (2011). Health care providers' mindfulness and treatment outcomes: a critical review of the research literature. Mindfulness, 2, 242-253.

Fekete, S. (1991). Risks of helping professions. Psychiatry. Hung, 6(1), 17-29.

Flook, L, Goldberg, S.B., Pinger, L., Bonus, K., \& Davidson, R.J. (2013). Mindfulness for teachers: A pilot study to assess effects on stress, burnout, and teaching efficacy. Mind, Brain, and Education, 7, 182-195.

Franco, C., Mańas, I., Cangas, A.J., Moreno, E., \& Gallego, J. (2010). Reducing teachers' psychological distress through a mindfulness training program. The Spanish Journal of Psychology, 13, 655-666.

Frank, J. L., Reibel, D., Broderick, P., Cantrell, T., \& Metz, S. (2015). The effectiveness of mindfulness-based stress reduction on educator stress and well-being: Results from a pilot study. Mindfulness, 6(2), 208-216. doi:10.1007/s12671-013-0246-2

Fries, E., Dettenborn, L., \& Kirschbaum, C. (2009). The cortisol awakening response (CAR): facts and future directions. International journal of Psychophysiology, 72(1), 67-73. 
Gabriel P. \& Liimatainen, M.R., (2000) Mental health in the workplace. Geneva, International Labour Organisation.

Gawrysiak, M., Nicholas, C., \& Hopko, D. R. (2009). Behavioral activation for moderately depressed university students: Randomized controlled trial. Journal of Counseling Psychology, 56(3), 468.

Geving, A.M. (2007). Identifying the types of student and teacher behaviors associated with teacher stress. Teaching and Teacher Education, 23, 624-640.

Gold, E., Smith, A., Hopper, I., Herne, D., Tansey, G., \& Hulland, C. (2010). Mindfulness-based stress reduction (MBSR) for primary school teachers. Journal of Child and Family Studies, 19, 184-189.

Greenglass, E.R., \& Burke, R.J. (2003). Teacher stress. In M.F. Dollard, A.H. Winefield, \& H.R. Winefield (Eds.), Occupational stress in the service professions (pp. 213236). New York: Taylor and Francis.

Greenglass, E., Fiksenbaum, L., \& Burke, R.J. (1996). Components of social support, buffering effects, and burnout: Implications for psychological functioning. Anxiety, Stress, and Coping: An International Journal, 9, 185-197.

Griffith, J., Steptoe, A., \& Cropley, M. (1999). An investigation of coping strategies associated with job stress in teachers. British Journal of Educational Psychology, 69(4), 517-531. 
Grossi, G., Perski, A., Ekstedt, M., Johansson, T., Lindström, M., \& Holm, K. (2005). The morning salivary cortisol response in burnout. Journal of psychosomatic research, 59(2), 103-111.

Grossman, P., Neimann, L., Schmidt, S., \& Walach, H. (2004). Mindfulness-based stress reduction and health benefits: A meta-analysis. Journal of Psychosomatic Research, 57, 35-43.

Gustafsson, P. E., Janlert, U., Theorell, T., Westerlund, H., \& Hammarström, A. (2011). Socioeconomic status over the life course and allostatic load in adulthood: results from the Northern Swedish Cohort. Journal of Epidemiology and Community Health, 65(11), 986-992.

Hakanen, J.J., Bakker, A.B., \& Schaufeli, W.B. (2006). Burnout and work engagement among teachers. Journal of School Psychology, 43, 495-513.

Harris, A.R., Jennings, P.A., Katz, D.A., Abenavoli, R.M., \& Greenberg, M.T. (2016). Promoting stress management and wellbeing in educators: Feasibility and efficacy of a school-based yoga and mindfulness intervention. Mindfulness, 7, 143-154.

Hayes, S. C., Wilson, K. G., Gifford, E. V., Follette, V. M., \& Strosahl, K. (1996). Experiential avoidance and behavioral disorders: A functional dimensional approach to diagnosis and treatment. Journal of Consulting and Clinical Psychology, 64(6), 1152. 
Henry, J. D., \& Crawford, J. R. (2005). The short-form version of the Depression Anxiety Stress Scales (DASS-21): Construct validity and normative data in a large nonclinical sample. British journal of clinical psychology, 44(2), 227-239.

Holm, S. (1979). A simple sequentially rejective multiple test procedure. Scandinavian journal of statistics, 65-70.

Huberman, M. (1993). The lives of teachers. London: Cassell.

Hwang, Y. S., Bartlett, B., Greben, M., \& Hand, K. (2017). A systematic review of mindfulness interventions for in-service teachers: A tool to enhance teacher wellbeing and performance. Teaching and Teacher Education, 64, 26-42.

ILO/UNESCO Joint Committee of Experts on the Application of the Recommendations Concerning the Status of Teachers (1994). Report, Geneva, July 12-20.

Insel, T., Cuthbert, B., Garvey, M., Heinssen, R., Pine, D. S., Quinn, K., ... \& Wang, P. (2010). Research domain criteria (RDoC): toward a new classification framework for research on mental disorders.

Irving, J.A., Dobkin, P.L., \& Park, J. (2009). Cultivating mindfulness in health care professionals: A review of empirical studies of mindfulness-based stress reduction (MBSR). Complementary Therapies in Clinical Practice, 15, 61-66.

Jennett, H.K., Harris, S.L., \& Mesibov, G.B. (2003). Commitment to philosophy, teacher efficacy, and burnout among teachers of children with autism. Journal of Autism and Developmental Disorders, 33, 583-593. 
Jennings, P. A. (2015). Early childhood teachers' well-being, mindfulness, and selfcompassion in relation to classroom quality and attitudes towards challenging students. Mindfulness, 6(4), 732-743.

Jennings, P.A., Frank, J.L., Snowberg, K.E., Coccia, M.A., \& Greenberg, M.T. (2013). Improving classroom learning environments by cultivating awareness and resilience in education (CARE); Results of a randomized controlled trial. School Psychology Quarterly, 28, 374-390.

Jennings, P. A., \& Greenberg, M. T. (2009). The prosocial classroom: Teacher social and emotional competence in relation to student and classroom outcomes. Review of Educational Research, 79(1), 491-525.

Jennings, P.A., Snowberg, K.E., Coccia, M.A., \& Greenberg, M.T. (2011). Improving classroom learning environments by cultivating awareness and resilience in education (CARE): Results of two pilot studies. Journal of Classroom Interaction, 46, 37-48.

Juster, R. P., Sindi, S., Marin, M. F., Perna, A., Hashemi, A., Pruessner, J. C., \& Lupien, S. J. (2011). A clinical allostatic load index is associated with burnout symptoms and hypocortisolemic profiles in healthy workers. Psychoneuroendocrinology, 36(6), 797-805.

Kabat-Zinn, J. (1994). Wherever you go, there you are. New York, NY: Hyperion. 
Kabat-Zinn, J. (1982). An outpatient program in behavioral medicine for chronic pain patients based on the practice of mindfulness medication: Theoretical considerations and preliminary results. General Hospital Psychiatry, 4, 33-47.

Kessler, R. C., Berglund, P., Demler, O., Jin, R., Koretz, D., Merikangas, K. R., ... \& Wang, P. S. (2003). The epidemiology of major depressive disorder: results from the National Comorbidity Survey Replication (NCS-R). JAMA: Journal of American Medical Association, 289(23), 3095-3105.

Kilpatrick, L.A., Suyenobu, B.Y., Smith, S.R., Bueller, J.A., Goodman, T., Creswell, J.D., ... Naliboff, B.D. (2011). Impact of mindfulness-based stress reduction training on intrinsic brain connectivity, Neuroimage, 56, 290-298.

Kim, J.S. (2008) Examining the effectiveness of solution focused brief therapy: A metaanalysis. Research on Social Work Practice, 18(2), 107-116.

Kirkwood, G., Rampes, H., Tuffrey, V., Richardson, J., \& Pilkington, K. (2005). Yoga for anxiety: A systematic review of the research literature. British Journal of Sports Medicine, 39, 884-891.

Klingbeil, D. A., \& Renshaw, T. L. (2018). Mindfulness-based interventions for teachers: A meta-analysis of the emerging evidence base. School Psychology Quarterly, $33(4), 501$.

Klusmann, U., Kunter, M., Trautwein, U., Lüdtke, O., \& Baumert, J. (2008). Teachers' occupational well-being and quality of instruction: The important role of selfregulatory patterns. Journal of Educational Psychology, 100(3), 702-715. 
Kohtala, A., Lappalainen, R., Savonen, L., Timo, E., \& Tolvanen, A. (2015). A foursession acceptance and commitment therapy based intervention for depressive symptoms delivered by masters degree level psychology students: A preliminary study. Behavioural and Cognitive Psychotherapy, 43(3), 360-373.

Kyllönen, H. M., Muotka, J., Puolakanaho, A., Astikainen, P., Keinonen, K., \& Lappalainen, R. (2018). A brief acceptance and commitment therapy intervention for depression: A randomized controlled trial with 3-year follow-up for the intervention group. Journal of Contextual Behavioral Science, 10, 55-63.

Kyriacou, C. (2001). Teacher stress: Directions for future research. Educational Review, 53(1), 27-35.

Kyriacou, C. (2000). Stressbusting for teachers. London, England: Nelson Thornes.

Kyriacou, C., \& Sutcliffe, J. (1979). Teacher stress and satisfaction. Educational Research, 21, 89-96.

Lambert, R.G., \& McCarthy, C.J. (Eds.). (2006). Understanding teacher stress in an era of accountability (Vol. 3). Greenwich, CT: Information Age.

Langelaan, S., Schaufeli, W. B., van Doornen, L. J., Bakker, A. B., \& van Rhenen, W. (2007). Is burnout related to allostatic load?. International journal of behavioral medicine, 14(4), 213-221.

Lazarus, R.S., \& Folkman, S. (1984). Stress, appraisal and coping. New York: Springer.

LeCompte, M.D., \& Dworkin, A.G. (1991). Giving up on school: Student dropouts and teacher burnouts. Newbury Park, CA: Corwin. 
Lomas, T., Medina, J. C., Ivtzan, I., Rupprecht, S., \& Eiroa-Orosa, F. J. (2017). The impact of mindfulness on the wellbeing and performance of educators: A systematic review of the empirical literature. Teaching and Teacher Education, $61,132-141$.

Lovibond, P. F., \& Lovibond, S. H. (1995). The structure of negative emotional states: Comparison of the Depression Anxiety Stress Scales (DASS) with the Beck Depression and Anxiety Inventories. Behaviour research and therapy, 33(3), 335343.

Lutz, A., Slagter, H.A., Dunne, J., \& Davidson, R.J. (2008). Attention regulation and monitoring in meditation. Trends in Cognitive Sciences, 12, 163-169.

Marzano, R. J., Marzano, J. S., \& Pickering, D. (2003). Classroom management that works: Research-based strategies for every teacher. ASCD.

Maslach, C., \& Jackson, S.E. (1981). Maslach burnout inventory manual. Mountain View, California: CPP, Inc.

Maslach, C., Jackson, S.E., \& Leiter, M.P. (1996). Maslach burnout inventory manual ( $3^{\text {rd }}$ ed.). Mountain View, California: CPP, Inc.

Maslach, C., Schaufeli, W.B., \& Leiter, M.P. (2001). Job burnout. Annual Review of Psychology, 52, 397-422.

Mattei, J., Demissie, S., Falcon, L. M., Ordovas, J. M., \& Tucker, K. L. (2010). Allostatic load is associated with chronic conditions in the Boston Puerto Rican Health 
Study. Social Science \& Medicine (1982), 70(12), 1988-1996.

http://doi.org/10.1016/j.socscimed.2010.02.024

McCarthy, C.J., \& Lambert, R.G. (2006). Helping teachers balance demands and resources in an era of accountability. In R. Lambert and C. McCarthy (Eds.), Understanding teacher stress in an age of accountability (pp. 215-226). Greenwich, CT: Information Age Publishing.

McCarthy, C.J., Lambert, R.C., O’Donnell, M., \& Melendres, L.T. (2009). The relation of elementary teachers' experience, stress, and coping resources to burnout symptoms. Elementary School Journal, 109, 282-300.

McEwen, B. S. (1998). Stress, adaptation, and disease: Allostasis and allostatic load. Annals of the New York academy of sciences, 840(1), 33-44.

McEwen, B. S. (2004). Protection and damage from acute and chronic stress: allostasis and allostatic overload and relevance to the pathophysiology of psychiatric disorders. Annals of the New York Academy of Sciences, 1032(1), 1-7.

McEwen, B. S., \& Gianaros, P. J. (2011). Stress-and allostasis-induced brain plasticity. Annual Review of Medicine, 62, 431-445.

Mearns, J., \& Cain, J. E. (2003). Relationships between teachers' occupational stress and their burnout and distress: Roles of coping and negative mood regulation expectancies. Anxiety, Stress \& Coping, 16(1), 71-82. 
Miller, G. E., Chen, E., \& Zhou, E. S. (2007). If it goes up, must it come down? Chronic stress and the hypothalamic-pituitary-adrenocortical axis in humans. Psychological bulletin, 133(1), 25.

Mitmansgruber, H., Beck, T. N., Höfer, S., \& Schüßler, G. (2009). When you don’t like what you feel: Experiential avoidance, mindfulness and meta-emotion in emotion regulation. Personality and Individual Differences, 46(4), 448-453.

Miyake, A., Friedman, N.P., Emerson, M.J., Witzki, A.H., \& Howerter, A. (2000). The unity and diversity of executive functions and their contributions to complex “frontal lobe" tasks: A variable analysis. Cognitive Psychology, 41, 49-100.

Moya-Albiol, L., Serrano, M. Á., \& Salvador, A. (2010). Burnout as an important factor in the psychophysiological responses to a work day in Teachers. Stress and Health, 26(5), 382-393.

Muraven, M., \& Baumeister, R. F. (2000). Self-regulation and depletion of limited resources: Does self-control resemble a muscle?. Psychological bulletin, 126(2), 247.

Neves de Jesus, S., \& Conboy, J. (2001). A stress management course to prevent teacher distress. International Journal of Educational Management, 15(3), 131-137.

Pilkington, K., Kirkwood, G., Rampes, H., \& Richadrson, J. (2005). Yoga for depression: The research evidence. Journal of Affective Disorders, 89, 13-24. 
Pruessner, J. C., Hellhammer, D. H., \& Kirschbaum, C. (1999). Burnout, perceived stress, and cortisol responses to awakening. Psychosomatic Medicine, 61(2), 197204.

Pruessner, J. C., Gaab, J., Hellhammer, D. H., Lintz, D., Schommer, N., \& Kirschbaum, C. (1997). Increasing correlations between personality traits and cortisol stress responses obtained by data aggregation. Psychoneuroendocrinology, 22(8), 615625.

Reiser, J. E., \& McCarthy, C. J. (2018). Preliminary investigation of a stress prevention and mindfulness group for teachers. Journal For Specialists In Group Work, 43(1), 2-34. doi:10.1080/01933922.2017.1338811

Reiser, J. E., Murphy, S. L., \& McCarthy, C. J. (2016). Stress prevention and mindfulness: A psychoeducational and support group for teachers. The Journal for Specialists in Group Work, 41(2), 117-139.

Renshaw, T.L., \& O’Malley, M.D. (2014). Cultivating mindfulness in students. In M.J. Furlong, R. Gilman, \& E.S. Huebner (Eds.), Handbook of positive psychology in the schools (2 ${ }^{\text {nd }}$ ed., pp. 245-259). New York, NY: Routledge.

Renshaw, T.L. (2012). Mindfulness-based practices for crisis prevention and intervention. In S.E. Brock \& S.R. Jimerson (Eds.), Handbook of school crisis prevention and intervention ( $2^{\text {nd }}$ ed., pp. 401-422). Bethesda, MA: National Association of School Psychologists. 
Rice, D. P. (1999). Economic costs of substance abuse, 1995. Proceedings of the Association of American Physicians, 111(2), 119-125.

Richardson, K. M., \& Rothstein, H. R. (2008). Effects of occupational stress management intervention programs: a meta-analysis. Journal of Occupational Health Psychology, 13(1), 69.

Roemer, L., \& Orsillo, S. M. (2003). Mindfulness: A promising intervention strategy in need of further study. Clinical Psychology: Science and Practice, 10(2), 172-178.

Roeser, R.W., Skinner, E., Beers, J., \& Jennings, P.A. (2012). Mindfulness training and teachers' professional development: An emerging area of research and practice. Child Development Perspectives, 6, 167-173.

Roeser, R.W., Schonert-Reichl, K.A., Jha, A., Cullen, M., Wallace, L., Wilensky, R., ...Harrision J. (2013). Mindfulness training and reductions in teacher stress and burnout: Results from two randomized, waitlist-control field trials. Journal of Educational Psychology, 105(3), 1-18.

Saleh, P. \& Shapiro, C. M. (2008). Disabled sleep and burnout: implications for longterm health. Journal of Psychosomatic Research, 65, 1-3

Schaufeli, W.B., \& Greenglass, E.R. (2001). Introduction to special issue on burnout and health. Psychology \& Health, 16, 501-510.

Schaufeli, W. B., Leiter, M. P., \& Maslach, C. (2009). Burnout: 35 years of research and practice. Career development international, 14(3), 204-220. 
Schaufeli, W. B., Maslach, C., \& Marek, T. (1993). Historical and conceptual development of burnout. Professional burnout: Recent developments in theory and research, 1-16.

Schaufeli, W., \& Enzmann, D. (1998). The burnout companion to study and practice: A critical analysis. CRC Press.

Schwarzer, R., \& Hallum, S. (2008). Perceived teacher self-efficacy as a predictor of job stress and burnout: Mediation analyses. Applied psychology, 57, 152-171.

Seeman, T. E., Singer, B. H., Rowe, J. W., Horwitz, R. I., \& McEwen, B. S. (1997). Price of adaptation — allostatic load and its health consequences: MacArthur studies of successful aging. Archives of Internal Medicine, 157(19), 2259-2268.

Selye, H. (1956). The stress of life.

Selye, H. (1974). Stress sans détresse. Lippincott.

Skaalvik, E. M., \& Skaalvik, S. (2010). Teacher self-efficacy and teacher burnout: A study of relations. Teaching and Teacher Education, 26(4), 1059-1069.

Smith, A., Brice, C., Collins, A., Matthews, V., \& McNamara, R. (2000). The scale of occupational stress: a further analysis of the impact of demographic factors and type of job. Contract report 311/2000. Health \& Safety Executive. Sudbury: HSE Books.

Sonnenschein, M., Mommersteeg, P. M., Houtveen, J. H., Sorbi, M. J., Schaufeli, W. B., \& van Doornen, L. J. (2007). Exhaustion and endocrine functioning in clinical 
burnout: an in-depth study using the experience sampling method. Biological psychology, 75(2), 176-184.

Stalder, T., Kirschbaum, C., Kudielka, B. M., Adam, E. K., Pruessner, J. C., Wüst, S., ... \& Miller, R. (2016). Assessment of the cortisol awakening response: expert consensus guidelines. Psychoneuroendocrinology, 63, 414-432.

Thomas, N., Clark, V., \& Lavery, J. (2003). Self-reported work and family stress of female primary teachers. Australian Journal of Education, 47, 73-87.

Thorn, L., Hucklebridge, F., Evans, P., \& Clow, A. (2006). Suspected non-adherence and weekend versus week day differences in the awakening cortisol response. Psychoneuroendocrinology, 31(8), 1009-1018.

Travers, C.J. (2001). Stress in teaching: Past, present, and future. In J. Dunham (Ed.), Stress in the workplace: Past, present, and future (pp. 130-163). Philadelphia, PA: Whurr.

Travers, C.J., \& Cooper, C.L. (1996). Teachers under pressure: Stress in the teaching profession. London: Routledge.

Van Horn, J. E., Schaufeli, W. B., \& Enzmann, D. (1999). Teacher Burnout and Lack of Reciprocity 1. Journal of Applied Social Psychology, 29(1), 91-108.

Virgili, M. (2013). Mindfulness-based interventions reduce psychological distress in working adults: A meta-analysis of intervention studies. Mindfulness, 6, 326-337. 
Wardenaar, K. J., Vreeburg, S. A., van Veen, T., Giltay, E. J., Veen, G., Penninx, B. W., \& Zitman, F. G. (2011). Dimensions of depression and anxiety and the hypothalamo-pituitary-adrenal axis. Biological Psychiatry, 69(4), 366-373.

Whipp, P.R., Tan, G., \& Yeo, P.T. (2007). Experienced physical education teachers reaching their "Use-by Date": Powerless and disrespected. Research Quarterly for Exercise and Sport, 78, 487-499.

Winters, J. M. (2012). Physiological measurement(s) of the stress response. In V. H. Rice (Ed.), Handbook of stress, coping, and health: Implications for nursing research, theory, and practice., 2nd ed. (pp. 97-124). Thousand Oaks, CA: Sage Publications, Inc. Retrieved from http://search.ebscohost.com.pallas2.tcl.sc.edu/login.aspx?direct=true \&db=psyh\& $\mathrm{AN}=2011-29300-005 \&$ site $=$ ehost-live

Wust, S., Wolf, J., Hellhammer, D. H., Federenko, I., Schommer, N., \& Kirschbaum, C. (2000). The cortisol awakening response-normal values and confounds. Noise and Health, 2(7), 79.

Zechmeister, I., Kilian, R., \& McDaid, D. (2008). Is it worth investing in mental health promotion and prevention of mental illness? A systematic review of the evidence from economic evaluations. BMC public health, 8(1), 20.

Żołnierczyk-Zreda, D. (2005). An intervention to reduce work-related burnout in teachers. International Journal of Occupational Safety and Ergonomics, 11(4), 423-430. 


\title{
APPENDIX A \\ INTERVENTION OUTCOMES MEASURES
}

Teacher Stress Inventory (TSI)

\author{
$1=$ No strength; not noticeable $\quad 2$ = Mild strength; barely noticeable \\ 3 = Medium strength; moderately noticeable $\quad 4$ = Great strength; very noticeable \\ 5 = Major strength; extremely noticeable
}

1. I easily overcommit myself.

$\begin{array}{lllll}1 & 2 & 3 & 4 & 5\end{array}$

2. I become impatient if others do things too slowly.

$\begin{array}{lllll}1 & 2 & 3 & 4 & 5\end{array}$

3. I have to try doing more than one thing at a time.

$\begin{array}{lllll}1 & 2 & 3 & 4 & 5\end{array}$

4. I have little time to relax/enjoy the time of day.

$\begin{array}{lllll}1 & 2 & 3 & 4 & 5\end{array}$

5. I think about unrelated matters during conversations.

$\begin{array}{lllll}1 & 2 & 3 & 4 & 5\end{array}$

6. I feel uncomfortable wasting time.

$\begin{array}{lllll}1 & 2 & 3 & 4 & 5\end{array}$

7. There isn't enough time to get things done.

$\begin{array}{lllll}1 & 2 & 3 & 4 & 5\end{array}$

8. I rush in my speech.

$\begin{array}{lllll}1 & 2 & 3 & 4 & 5\end{array}$

9. There is little time to prepare for my lessons/responsibilities. $\quad \begin{array}{lllllll} & 1 & 2 & 3 & 4 & 5\end{array}$

10. There is too much work to do.

$\begin{array}{lllll}1 & 2 & 3 & 4 & 5\end{array}$

11. The pace of the school day is too fast.

$\begin{array}{lllll}1 & 2 & 3 & 4 & 5\end{array}$

12. My caseload/class is too big.

$\begin{array}{lllll}1 & 2 & 3 & 4 & 5\end{array}$

13. My person priorities are being shortchanged due to time demands.

14. There is too much administrative paperwork in my job.

15. I lack promotion and/or advancement in opportunities.

$\begin{array}{lllll}1 & 2 & 3 & 4 & 5\end{array}$

16. I am not progressing in my job as rapidity as I would like to. $\quad \begin{array}{lllllll}1 & 2 & 3 & 4 & 5\end{array}$

17. I need more status and respect in my job. $\quad 1 \quad 2 \quad 3 \quad 4 \quad 5$

18. I receive an inadequate salary for the work I do. $\quad 1 \quad \begin{array}{lllll} & 2 & 3 & 4 & 5\end{array}$

19. I lack recognition for the extra work and/or good teaching I do. $\begin{array}{llllll}1 & 2 & 3 & 4 & 5\end{array}$

Ifeel frustrated...

20. ...because of the discipline problems in my classroom. $\quad \begin{array}{lllll}1 & 2 & 3 & 4 & 5\end{array}$ 
21. ...having to monitor pupil behavior.

$\begin{array}{lllll}1 & 2 & 3 & 4 & 5\end{array}$

22. ...because some students would do better if they tried.

$\begin{array}{lllll}1 & 2 & 3 & 4 & 5\end{array}$

23. ....attempting to teach students who are poorly motivated.

$\begin{array}{lllll}1 & 2 & 3 & 4 & 5\end{array}$

24. ...because of inadequately/poorly defined discipline problems. $\begin{array}{llllll}1 & 2 & 3 & 4 & 5\end{array}$

25. ...when my authority is rejected by pupils/administration. $\quad \begin{array}{lllll}1 & 2 & 3 & 4 & 5\end{array}$

26. My personal opinions are not sufficiently aired.

$\begin{array}{lllll}1 & 2 & 3 & 4 & 5\end{array}$

27.

I lack control over decisions made about classroom/school matters.

$\begin{array}{lllll}1 & 2 & 3 & 4 & 5\end{array}$

28. I am not emotionally/intellectually stimulated on the job.

$\begin{array}{lllll}1 & 2 & 3 & 4 & 5\end{array}$

29. I lack opportunities for professional improvement.

$\begin{array}{lllll}1 & 2 & 3 & 4 & 5\end{array}$

I respond to stress...

30. ...by feeling insecure.

$\begin{array}{lllll}1 & 2 & 3 & 4 & 5\end{array}$

31. ...by feeling vulnerable.

$\begin{array}{lllll}1 & 2 & 3 & 4 & 5\end{array}$

32. ...by feeling unable to cope.

$\begin{array}{lllll}1 & 2 & 3 & 4 & 5\end{array}$

33. ...by feeling depressed.

$\begin{array}{lllll}1 & 2 & 3 & 4 & 5\end{array}$

34. ...by feeling anxious.

$\begin{array}{lllll}1 & 2 & 3 & 4 & 5\end{array}$

35. ...by sleeping more than normal.

$\begin{array}{lllll}1 & 2 & 3 & 4 & 5\end{array}$

36. ...by procrastinating.

$\begin{array}{lllll}1 & 2 & 3 & 4 & 5\end{array}$

37. ...by become fatigued in a very short time.

$\begin{array}{lllll}1 & 2 & 3 & 4 & 5\end{array}$

38. ...with physical exhaustion.

$\begin{array}{lllll}1 & 2 & 3 & 4 & 5\end{array}$

39. ...with physical weakness.

$\begin{array}{lllll}1 & 2 & 3 & 4 & 5\end{array}$

40. ...with feelings of increased blood pressure.

$\begin{array}{lllll}1 & 2 & 3 & 4 & 5\end{array}$

41. ...with feelings of heart pounding or racing.

$\begin{array}{lllll}1 & 2 & 3 & 4 & 5\end{array}$

42. ...with rapid and/or shallow breath.

$\begin{array}{lllll}1 & 2 & 3 & 4 & 5\end{array}$

43. ...with stomach pain of extended duration.

$\begin{array}{lllll}1 & 2 & 3 & 4 & 5\end{array}$

44. ...with stomach cramps.

$\begin{array}{lllll}1 & 2 & 3 & 4 & 5\end{array}$

45. ...with stomach acid.

$\begin{array}{lllll}1 & 2 & 3 & 4 & 5\end{array}$

46. ...by using over-the-counter drugs.

$\begin{array}{lllll}1 & 2 & 3 & 4 & 5\end{array}$

47. ...by using prescription drugs.

$\begin{array}{lllll}1 & 2 & 3 & 4 & 5\end{array}$

48. ...by using alcohol.

$\begin{array}{lllll}1 & 2 & 3 & 4 & 5\end{array}$

49. ...by calling in sick.

$\begin{array}{lllll}1 & 2 & 3 & 4 & 5\end{array}$ 


\section{Maslach Burnout Inventory - Educator's Survey (MBI-ES)}

\begin{tabular}{|c|c|c|}
\hline $0=$ Never & times a year or less & $2=$ Once a month or \\
\hline $3=\mathrm{A}$ few times a month & $\begin{array}{r}4=\text { Once a week } \\
\text { Every day }\end{array}$ & $5=\mathrm{A}$ few times a week \\
\hline
\end{tabular}

1. I feel emotionally drained from my work.

2. I feel used up at the end of the workday.

3. I feel fatigued when I get up in the morning and have to

3. face another day on the job.

4. I can easily understand how my students feel about things.

5. I feel I treat some students as if they were impersonal

5. objects.

6. Working with people all day is really a strain for me.

$\begin{array}{lllllll}0 & 1 & 2 & 3 & 4 & 5 & 6 \\ 0 & 1 & 2 & 3 & 4 & 5 & 6 \\ 0 & 1 & 2 & 3 & 4 & 5 & 6 \\ 0 & 1 & 2 & 3 & 4 & 5 & 6 \\ 0 & 1 & 2 & 3 & 4 & 5 & 6 \\ 0 & 1 & 2 & 3 & 4 & 5 & 6 \\ 0 & 1 & 2 & 3 & 4 & 5 & 6 \\ 0 & 1 & 2 & 3 & 4 & 5 & 6 \\ 0 & 1 & 2 & 3 & 4 & 5 & 6\end{array}$

7. I deal very effectively with the problems of my students.

8. I feel burnout out from my work.

9. I feel I'm positively influencing other people's lives through my work.

10. I've become more callous toward people since I took this job.

11. I worry this job is hardening me emotionally.

12. I feel very energetic.

13. I feel frustrated by my job.

14. I feel I'm working too hard on my job.

15. I don't really care what happens to some students.

16. Working with people directly puts too much stress on me.

$\begin{array}{lllllll}0 & 1 & 2 & 3 & 4 & 5 & 6\end{array}$

I can easily create a relaxed atmosphere with my

17. I can easily

$\begin{array}{lllllll}0 & 1 & 2 & 3 & 4 & 5 & 6\end{array}$

$\begin{array}{lllllll}0 & 1 & 2 & 3 & 4 & 5 & 6\end{array}$

$\begin{array}{lllllll}0 & 1 & 2 & 3 & 4 & 5 & 6\end{array}$

$\begin{array}{lllllll}0 & 1 & 2 & 3 & 4 & 5 & 6\end{array}$

$\begin{array}{lllllll}0 & 1 & 2 & 3 & 4 & 5 & 6\end{array}$

$\begin{array}{lllllll}0 & 1 & 2 & 3 & 4 & 5 & 6\end{array}$

18. I fee exhilarated after working closely with my students.

19. I have accomplished many worthwhile things in this job.

20. I feel like I'm at the end of my rope.

$\begin{array}{lllllll}0 & 1 & 2 & 3 & 4 & 5 & 6\end{array}$

21. In my work, I deal with emotional problems very calmly.

22. I feel students blame me for some of their problems.

$\begin{array}{lllllll}0 & 1 & 2 & 3 & 4 & 5 & 6\end{array}$

$\begin{array}{lllllll}0 & 1 & 2 & 3 & 4 & 5 & 6\end{array}$

$\begin{array}{lllllll}0 & 1 & 2 & 3 & 4 & 5 & 6\end{array}$

$\begin{array}{lllllll}0 & 1 & 2 & 3 & 4 & 5 & 6\end{array}$

$\begin{array}{lllllll}0 & 1 & 2 & 3 & 4 & 5 & 6\end{array}$ 


\section{Symptom Assessment - 45 (SA-45)}

$$
\begin{gathered}
1=\text { Not at all } \quad 2=\text { A little bit } \quad 3=\text { Moderately } \\
4=\text { Quite a bit } \quad 5=\text { Extremely }
\end{gathered}
$$

Please describe how much each problem has bothered or distressed you during the past 7 days, including today:

1. Feeling lonely.

2. Feeling blue.

3. Feeling no interested in things.

4. Feeling fearful.

5. The idea that someone else can control your thoughts.

6. Feeling others are to blame for most of your troubles.

7. Feeling afraid in open spaces or on the streets.

8. Hearing voices that other people do not hear.

9. Feeling that most people cannot be trusted.

10. Suddenly scared for no reason.

11. Temper outbursts that you could not control.

12. Feeling afraid to go out of your house alone.

13. Other people being aware of your private thoughts.

14. Feeling others do not understand you or are unsympathetic.

15. Feeling that people are unfriendly or dislike you.

16. Having to do things very slowly to ensure correctness.

17. Feeling inferior to others.

18. Soreness of your muscles.

19. Feeling that you are watched or talked about by others.

20. Having to check and double-check what you do.

21. Difficulty making decisions.

22. Feeling afraid to travel on buses, subways, or trains.

23. Hot or cold spells.

24. Having to avoid certain things, places, or activities because they frighten you.

25. Your mind going blank.

26. Numbness or tingling in parts of your body.

27. Feeling hopeless about the future.

28. Trouble concentrating.

29. Feeling weak in parts of your body.

30. Feeling tense or keyed up.

31. Heavy feelings in your arms or legs.

$\begin{array}{lllll}1 & 2 & 3 & 4 & 5 \\ 1 & 2 & 3 & 4 & 5 \\ 1 & 2 & 3 & 4 & 5 \\ 1 & 2 & 3 & 4 & 5 \\ 1 & 2 & 3 & 4 & 5 \\ 1 & 2 & 3 & 4 & 5 \\ 1 & 2 & 3 & 4 & 5 \\ 1 & 2 & 3 & 4 & 5 \\ 1 & 2 & 3 & 4 & 5 \\ 1 & 2 & 3 & 4 & 5 \\ 1 & 2 & 3 & 4 & 5 \\ 1 & 2 & 3 & 4 & 5 \\ 1 & 2 & 3 & 4 & 5 \\ 1 & 2 & 3 & 4 & 5 \\ 1 & 2 & 3 & 4 & 5 \\ 1 & 2 & 3 & 4 & 5 \\ 1 & 2 & 3 & 4 & 5 \\ 1 & 2 & 3 & 4 & 5 \\ 1 & 2 & 3 & 4 & 5 \\ 1 & 2 & 3 & 4 & 5 \\ 1 & 2 & 3 & 4 & 5 \\ 1 & 2 & 3 & 4 & 5 \\ 1 & 2 & 3 & 4 & 5 \\ 1 & 2 & 3 & 4 & 5 \\ 1 & 2 & 3 & 4 & 5 \\ 1 & 2 & 3 & 4 & 5 \\ 1 & 2 & 3 & 4 & 5 \\ 1 & 2 & 3 & 4 & 5 \\ 1 & 2 & 3 & 4 & 5 \\ 1 & 2 & 3 & 4 & 5 \\ 1 & 2 & 3 & 4 & 5\end{array}$


32. Feeling uneasy when people are watching or talking to you. $\quad \begin{array}{llllllllll}1 & 2 & 3 & 4 & 5\end{array}$

33. Having thoughts that are not your own. $\quad 1 \quad 2 \quad 3 \quad 4 \quad 5$

34. Having urges to beat, injure, or harm someone. $\quad \begin{array}{lllll} & 2 & 3 & 4 & 5\end{array}$

35. Having urges to break or smash things. $\quad 1 \begin{array}{lllll} & 2 & 3 & 4 & 5\end{array}$

36. Feeling very self-conscious with others. $\quad 1 \begin{array}{lllll} & 2 & 3 & 4 & 5\end{array}$

37. Feeling uneasy in crowds, such as shopping or at a movie. $\quad \begin{array}{lllllll}1 & 2 & 3 & 4 & 5\end{array}$

38. Spells of terror or panic. $\quad \begin{array}{lllll}1 & 2 & 3 & 4 & 5\end{array}$

39. Getting into frequent arguments. $\quad \begin{array}{lllll} & 2 & 3 & 4 & 5\end{array}$

40. Others not giving you proper credit for your achievements. $\quad \begin{array}{llllll}1 & 2 & 3 & 4 & 5\end{array}$

41. Feeling so restless you couldn't sit still. $\quad 1 \quad \begin{array}{lllll} & 2 & 3 & 4 & 5\end{array}$

42. Feelings of worthlessness. $\quad \begin{array}{lllll}1 & 2 & 3 & 4 & 5\end{array}$

43 Shouting or throwing things. $\quad \begin{array}{lllll}1 & 2 & 3 & 4 & 5\end{array}$

44. Feeling that people will take advantage of your if you let them. $\begin{array}{llllll}1 & 2 & 3 & 4 & 5\end{array}$

45. The idea that you should be punished for your sins. $\quad 1 \quad 2 \quad 3 \quad 4 \quad 5$ 


\section{APPENDIX B}

\section{MECHANISMS OF CHANGE MEASURE}

\section{Five-Facet Mindfulness Questionnaire (FFMQ)}
$1=$ Never or very rarely true
$2=$ Rarely true
$3=$ Sometimes true
$4=$ Often true
$5=$ Very often or always true

1. When I'm talking, I deliberately notice the sensation of my body moving.

2. I'm good at finding words to describe my feelings.

$\begin{array}{lllll}1 & 2 & 3 & 4 & 5\end{array}$

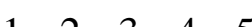

4. I perceive my feeling and emotions without having to react to them.

5. When I do things, my mind wanders off and I'm easily distracted.

$\begin{array}{lllll}1 & 2 & 3 & 4 & 5\end{array}$

When I take a shower or bath, I stay alert to the sensation of

6. water on my body.

$\begin{array}{lllll}1 & 2 & 3 & 4 & 5\end{array}$

7. I can easily put my beliefs, opinions, and expectations into words.

$\begin{array}{lllll}1 & 2 & 3 & 4 & 5\end{array}$

8. I don't pay attention to what I'm doing because I'm daydreaming.

9. I watch my feelings without getting lost in them.

10. I tell myself I shouldn't be feeling the way I'm feeling.

11. I notice how foods and drinks affect my thoughts, bodily sensations, and emotions.

12. It's hard for me to find the words to describe what I'm feeling. $\quad \begin{array}{lllll}1 & 2 & 3 & 4 & 5\end{array}$

13. I am easily distracted.

14. I believe some of my thoughts or abnormal or bad and I shouldn't think that way.

15. I pay attention to sensations, such as the wind in my hair or sun on my face.

$\begin{array}{lllll}1 & 2 & 3 & 4 & 5\end{array}$

$\begin{array}{lllll}1 & 2 & 3 & 4 & 5\end{array}$

$\begin{array}{lllll}1 & 2 & 3 & 4 & 5\end{array}$

$\begin{array}{lllll}1 & 2 & 3 & 4 & 5\end{array}$

I have trouble thinking of the right words to express how I feel about things.

$\begin{array}{lllll}1 & 2 & 3 & 4 & 5\end{array}$

17. I make judgments about whether my thoughts are good or bad. I find it difficult to stay focused on what's happening in the

18. present.

$\begin{array}{lllll}1 & 2 & 3 & 4 & 5\end{array}$

$\begin{array}{lllll}1 & 2 & 3 & 4 & 5\end{array}$ 
When I have distressing thoughts or images, I "step back" and

19. am aware of the thought or image without getting taken over by $\begin{array}{lllll}1 & 2 & 3 & 4 & 5\end{array}$ it.

20. I pay attention to sounds, such as clocks ticking, birds chirping, or cars passing.

21. In difficult situations, I can pause without immediately reacting. $\quad \begin{array}{lllll}1 & 2 & 3 & 4 & 5\end{array}$ When I have a sensation in my body, it's difficult for me to describe it because I can't find the right words.

$\begin{array}{lllll}1 & 2 & 3 & 4 & 5\end{array}$

It seems I am "running on automatic" without much awareness

23. of what I'm doing.

$\begin{array}{lllll}1 & 2 & 3 & 4 & 5\end{array}$

When I have distressing thoughts or images, I feel calm soon

24. after.

$\begin{array}{lllll}1 & 2 & 3 & 4 & 5\end{array}$

25. I tell myself that I shouldn't be thinking the way I'm thinking. $\quad \begin{array}{lllll}1 & 2 & 3 & 4 & 5\end{array}$

26. I notice the smells and aromas of things. $\quad 1 \quad 2 \quad 3 \quad 4 \quad 5$

27. Even when I'm feeling terribly upset, I can find a way to put it into words.

$\begin{array}{lllll}1 & 2 & 3 & 4 & 5\end{array}$

28. I rush through activities without being really attentive to them. $\quad \begin{array}{lllll}1 & 2 & 3 & 4 & 5\end{array}$

29. When I have distressing thoughts or images, I am able to just notice them without reacting.

$\begin{array}{lllll}1 & 2 & 3 & 4 & 5\end{array}$

I think some of my emotions are bad or inappropriate and I

30 . shouldn't feel them.

$\begin{array}{lllll}1 & 2 & 3 & 4 & 5\end{array}$

31. I notice visual elements in art of nature, such as colors, shapes, textures, or patterns of light and shadow.

32. My natural tendency is to put my experience into words.

33.

When I have distressing thoughts or images, I just notice them and let go.

$\begin{array}{lllll}1 & 2 & 3 & 4 & 5\end{array}$

$\begin{array}{lllll}1 & 2 & 3 & 4 & 5\end{array}$

$\begin{array}{lllll}1 & 2 & 3 & 4 & 5\end{array}$

I do jobs or tasks automatically without being aware of what I'm

doing.

$\begin{array}{lllll}1 & 2 & 3 & 4 & 5\end{array}$

When I have distressing thoughts or images, I judge myself as

35. good or bad, depending on what the thought/image is about.

$\begin{array}{lllll}1 & 2 & 3 & 4 & 5\end{array}$

I pay attention to how my emotions affect my thoughts and

behavior.

$\begin{array}{lllll}1 & 2 & 3 & 4 & 5\end{array}$

37. I can usually describe how I feel at the moment in considerable detail.

38. I find myself doing thing without paying attention.

39. I disapprove of myself when I have irrational fears.

$\begin{array}{lllll}1 & 2 & 3 & 4 & 5\end{array}$

$\begin{array}{lllll}1 & 2 & 3 & 4 & 5\end{array}$

$\begin{array}{lllll}1 & 2 & 3 & 4 & 5\end{array}$ 